\title{
Chromene-annulated Bacteriochlorins
}

Michael A. Hyland, Nisansala Hewage, Kimberly Walton, Arunpatcha Nimthong Roldan, Mathias Zeller, Milinda Samaraweera, José A. Gascon, and Christian Brückner*

Department of Chemistry, University of Connecticut, Storrs, CT 06269-3060, United States and and Department of Chemistry, Youngstown State University, One University Plaza, Youngstown, Ohio 44555-3663

* Author to whom correspondence should be addressed: Fax: (+1) 860 486-2981; Tel: (+1) 860 486-2743; e-mail: c.bruckner@uconn.edu

\section{TABLE OF CONTENTS}

Figure S1. UV-vis absortion (solid red trace) and fluorescence emission (broken black trace)

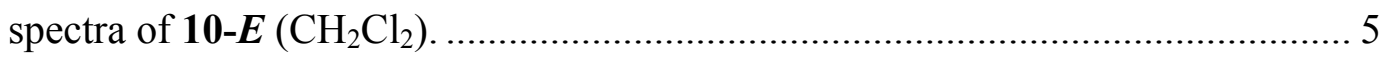

Figure S2. UV-vis absortion (solid red trace) and fluorescence emission (broken black trace)

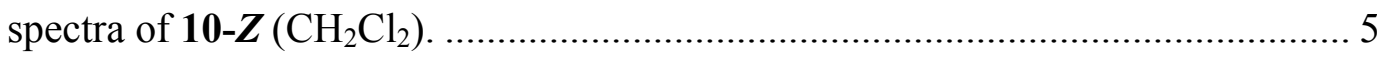

Figure S3. UV-vis absortion (solid red trace) and fluorescence emission (broken black trace)

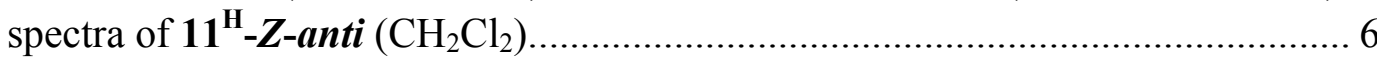

Figure S4. UV-vis absortion (solid red trace) and fluorescence emission (broken black trace)

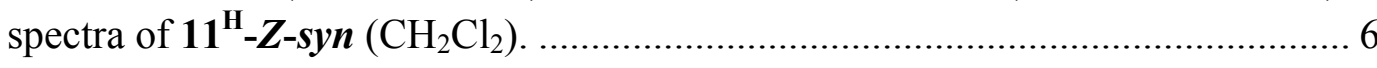

Figure S5. UV-vis absortion (solid red trace) and fluorescence emission (broken black trace)

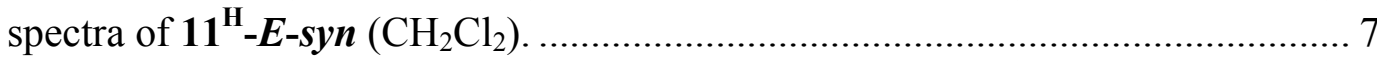

Figure S6. UV-vis absortion (solid red trace) and fluorescence emission (broken black trace)

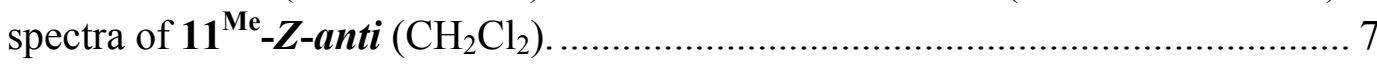


Figure S7. UV-vis absortion (solid red trace) and fluorescence emission (broken black trace) spectra of $11^{\mathrm{Me}}-\boldsymbol{Z}$-syn $\left(\mathrm{CH}_{2} \mathrm{Cl}_{2}\right)$.

Figure S8. UV-vis absortion (solid red trace) and fluorescence emission (broken black trace)

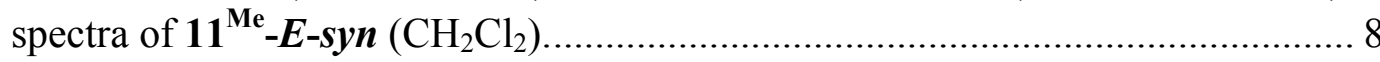

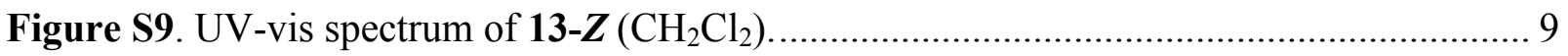

Figure S10. UV-vis absortion (solid red trace) and fluorescence emission (broken black trace)

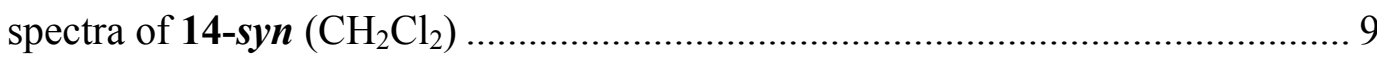

Figure S11. UV-vis spectra of $\mathbf{1 4}^{\mathrm{Me}}$ in neutral $\left(\mathrm{CH}_{2} \mathrm{Cl}_{2}\right.$; solid red trace) and acidified $\left(\mathrm{CH}_{2} \mathrm{Cl}_{2}\right.$ with $\sim 0.1 \%$ of TFA; broken black trace) media.

Figure S12. UV-vis spectra of $14^{\mathrm{Et}}$ in neutral $\left(\mathrm{CH}_{2} \mathrm{Cl}_{2}\right.$; solid red trace $)$ and acidified $\left(\mathrm{CH}_{2} \mathrm{Cl}_{2}\right.$ with $\sim 0.1 \%$ of TFA; broken black trace) media. ........................................ 10

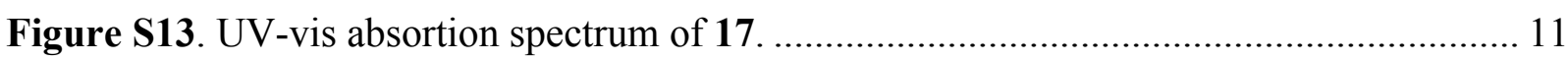

Figure S14. UV-vis absortion (solid red trace) and fluorescence emission (broken black trace)

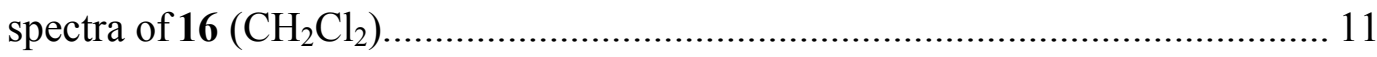

${ }^{1} \mathrm{H},{ }^{13} \mathrm{C}$, and ${ }^{19} \mathrm{~F}$ NMR Spectra of the Compounds Described ..................................................... 12

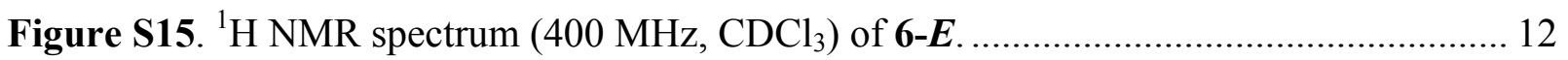

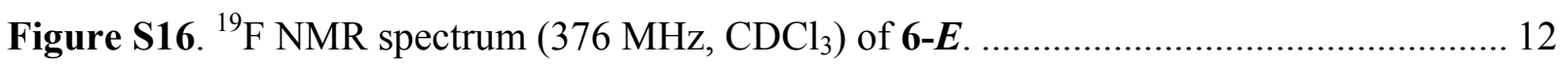

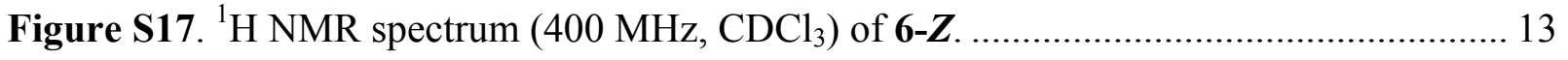

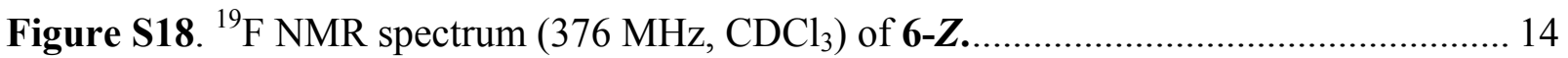

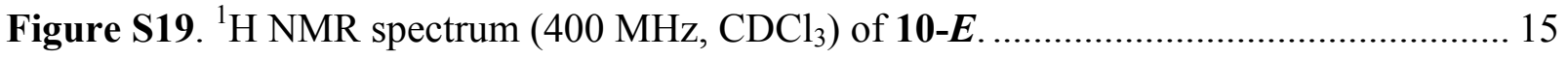

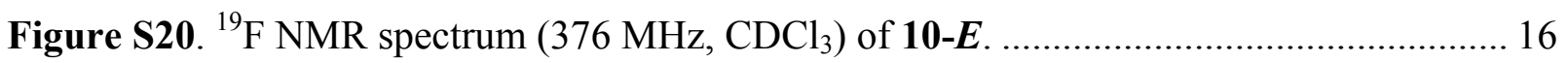

Figure S21. ${ }^{19} \mathrm{~F},{ }^{19} \mathrm{~F}$ QF-COSY NMR spectrum $\left(376 \mathrm{MHz}, \mathrm{CDCl}_{3}\right.$ ) of 10-E..................... 17

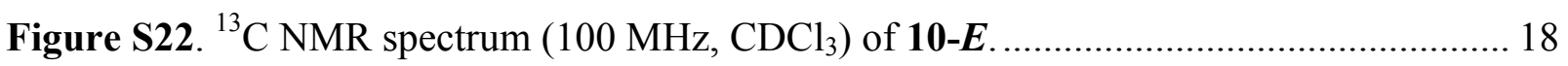

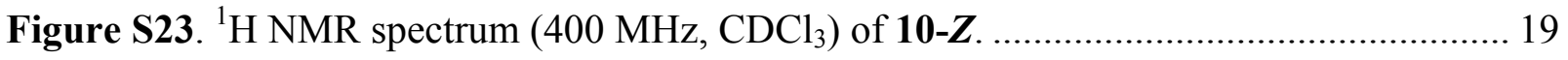

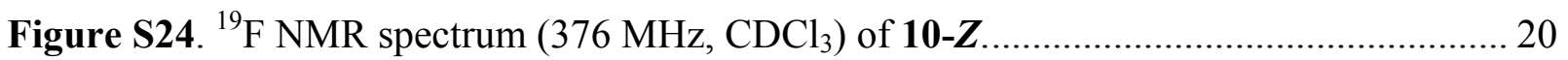

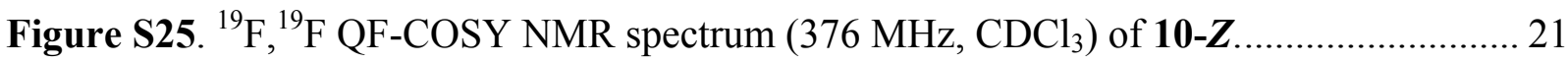

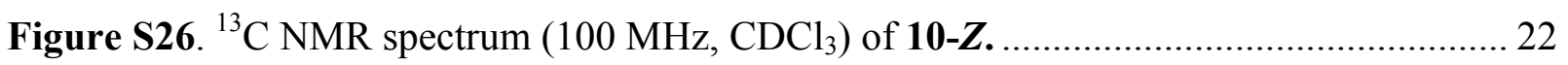

Figure S27. ${ }^{1} \mathrm{H}$ NMR spectrum $\left(400 \mathrm{MHz}, \mathrm{CDCl}_{3}\right)$ of $\mathbf{1 1}^{\mathbf{H}}$-Z-anti. ................................. 23

Figure S28. ${ }^{19} \mathrm{~F}$ NMR spectrum $\left(376 \mathrm{MHz}, \mathrm{CDCl}_{3}\right)$ of $\mathbf{1 1}^{\mathbf{H}}$-Z-anti.................................. 24

Figure S29. ${ }^{19} \mathrm{~F},{ }^{19} \mathrm{~F}$ QF-COSY NMR spectrum $\left(376 \mathrm{MHz}, \mathrm{CDCl}_{3}\right)$ of $\mathbf{1 1}^{\mathbf{H}}-\boldsymbol{Z}$-anti............... 25

Figure S30. ${ }^{1} \mathrm{H},{ }^{1} \mathrm{H}$ COSY NMR spectrum $\left(400 \mathrm{MHz}, \mathrm{CDCl}_{3}\right)$ of $\mathbf{1 1}^{\mathbf{H}}$-Z-anti...................... 26 


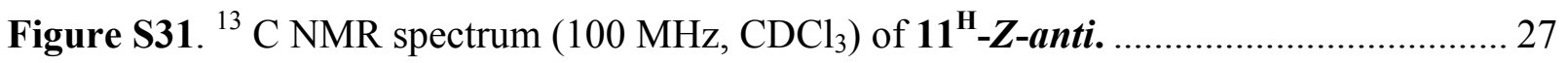

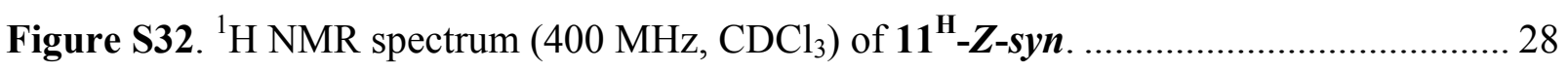

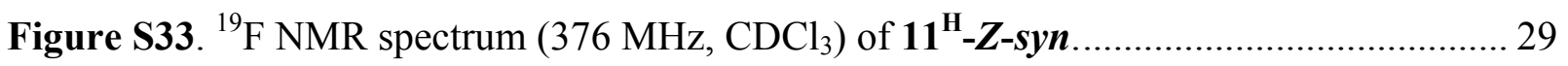

Figure S34. ${ }^{19} \mathrm{~F},{ }^{19} \mathrm{~F}$ QF-COSY NMR spectrum $\left(376 \mathrm{MHz}, \mathrm{CDCl}_{3}\right)$ of $\mathbf{1 1}^{\mathrm{H}}$-Z-syn .................. 30

Figure S35. ${ }^{1} \mathrm{H},{ }^{1} \mathrm{H}$ COSY NMR spectrum $\left(400 \mathrm{MHz}, \mathrm{CDCl}_{3}\right)$ of $\mathbf{1 1}^{\mathrm{H}}$-Zz-syn ........................... 31

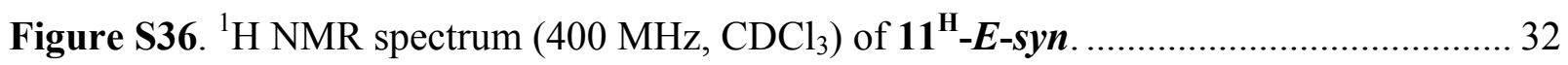

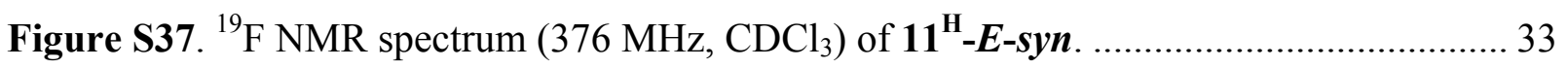

Figure S38. ${ }^{19} \mathrm{~F},{ }^{19} \mathrm{~F}$ QF-COSY NMR spectrum $\left(376 \mathrm{MHz}, \mathrm{CDCl}_{3}\right)$ of $\mathbf{1 1}^{\mathbf{H}}$-E-syn. .................. 34

Figure S39. ${ }^{1} \mathrm{H},{ }^{1} \mathrm{H}$ COSY NMR spectrum $\left(400 \mathrm{MHz}, \mathrm{CDCl}_{3}\right)$ of $\mathbf{1 1}^{\mathbf{H}}$-E-syn ......................... 35

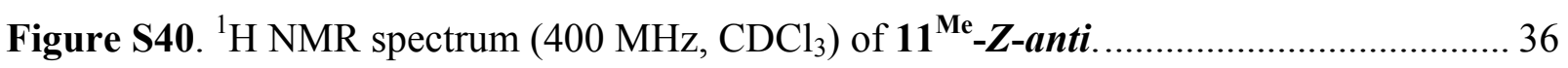

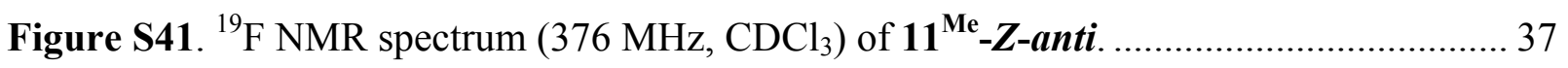

Figure S42. ${ }^{1} \mathrm{H},{ }^{1} \mathrm{H}-\mathrm{COSY}$ NMR spectrum $\left(400 \mathrm{MHz}, \mathrm{CDCl}_{3}\right)$ of $\mathbf{1 1}{ }^{\mathrm{Me}}-\mathbf{Z}$-anti........................ 38

Figure S43. ${ }^{1} \mathrm{H},{ }^{19} \mathrm{~F}-\mathrm{HOESY}$ NMR spectrum $\left(400,376 \mathrm{MHz}, \mathrm{CDCl}_{3}\right)$ of $\mathbf{1 1}^{\mathbf{M e}}-$ Z-anti............ 39

Figure S44. Partial ${ }^{1} \mathrm{H},{ }^{19} \mathrm{~F}-\mathrm{HOESY}$ NMR spectrum $\left(400,376 \mathrm{MHz}, \mathrm{CDCl}_{3}\right)$ of $\mathbf{1 1}{ }^{\mathrm{Me}}$-Z-anti. 40

Figure S45. ${ }^{1} \mathrm{H}$ NMR spectrum $\left(400 \mathrm{MHz}, \mathrm{CDCl}_{3}\right)$ of $\mathbf{1 1}^{\mathrm{Me}}$-Z-syn ......................................... 41

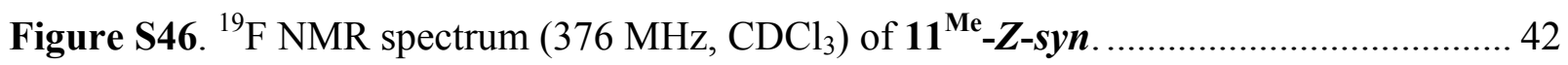

Figure S47. ${ }^{1} \mathrm{H},{ }^{1} \mathrm{H}-\mathrm{COSY}$ NMR spectrum $\left(400 \mathrm{MHz}, \mathrm{CDCl}_{3}\right)$ of $\mathbf{1 1}^{\mathrm{Me}}-\boldsymbol{Z}$-syn. ........................ 43

Figure S48. ${ }^{1} \mathrm{H}$ NMR spectrum $\left(400 \mathrm{MHz}, \mathrm{CDCl}_{3}\right)$ of $\mathbf{1 1}^{\mathrm{Me}}-\boldsymbol{E}$-syn ..................................... 44

Figure S49. ${ }^{19} \mathrm{~F}$ NMR spectrum $\left(376 \mathrm{MHz}, \mathrm{CDCl}_{3}\right.$ ) of $\mathbf{1 1}^{\mathrm{Me}}$-E-syn....................................... 45

Figure S50. ${ }^{1} \mathrm{H},{ }^{1} \mathrm{H}-\mathrm{COSY}$ NMR spectrum $\left(400 \mathrm{MHz}, \mathrm{CDCl}_{3}\right)$ of $\mathbf{1 1}^{\mathrm{Me}}$-E-syn....................... 46

Figure S51. ${ }^{13} \mathrm{C}$ NMR spectrum $\left(100 \mathrm{MHz}, \mathrm{CDCl}_{3}\right)$ of $\mathbf{1 1}^{\mathrm{Me}}$-E-syn ...................................... 47

Figure S52. ${ }^{1} \mathrm{H},{ }^{19} \mathrm{~F}-\mathrm{HOESY}$ NMR spectrum $\left(400,376 \mathrm{MHz}, \mathrm{CDCl}_{3}\right)$ of $\mathbf{1 1}^{\mathrm{Me}}$-E-syn ............ 48

Figure S53. ${ }^{13} \mathrm{C}$ NMR spectrum $\left(100 \mathrm{MHz}, \mathrm{CDCl}_{3}\right)$ of $\mathbf{1 1}^{\mathrm{Me}}$-E-syn .................................... 49

Figure S54. ${ }^{1} \mathrm{H}$ NMR spectrum ( $400 \mathrm{MHz}, \mathrm{CDCl}_{3}$ ) of 14-syn .......................................... 50

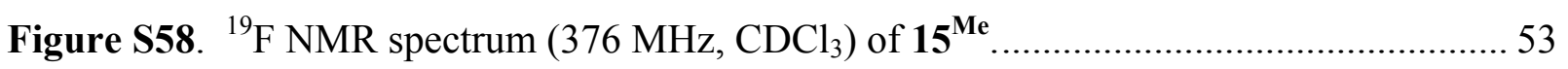

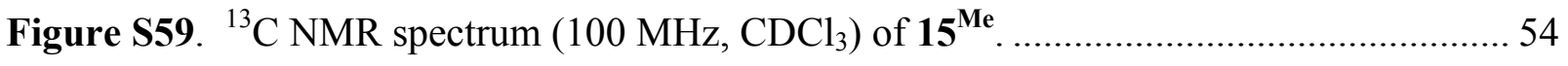

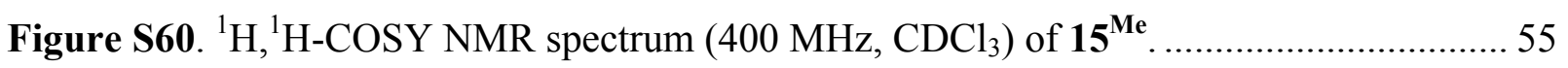

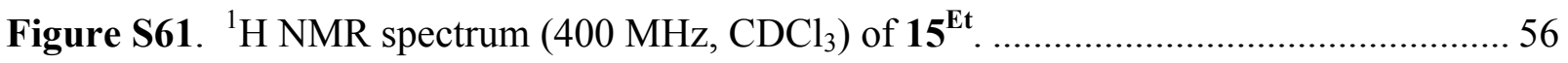

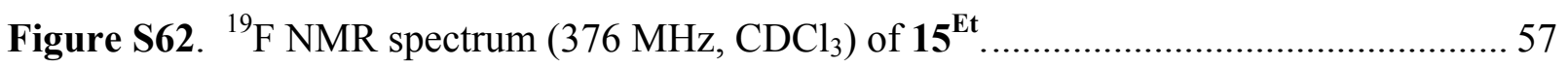

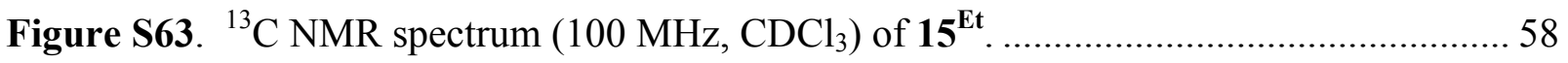




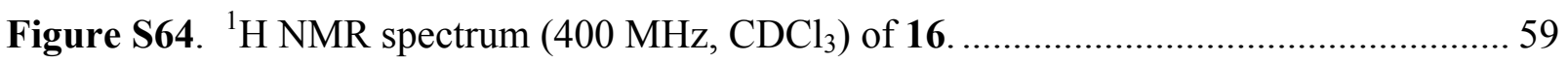

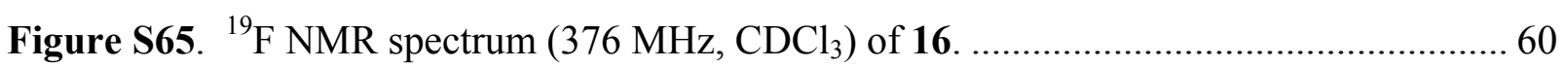

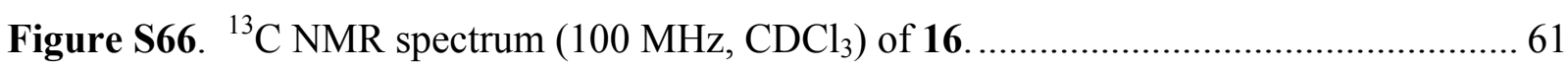

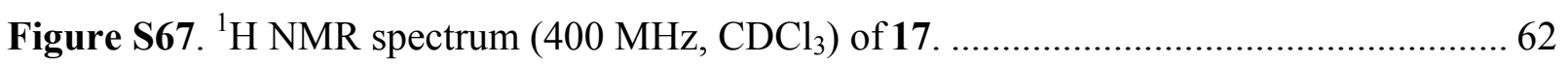

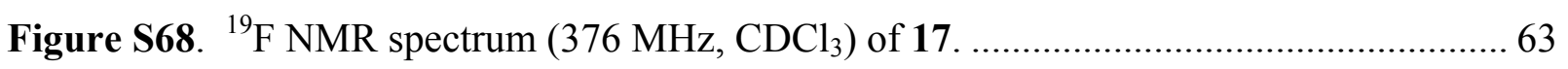

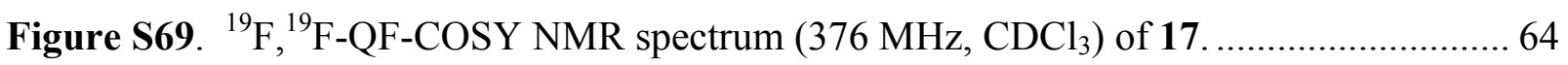

Table S1. Experimental details to the Crystal Structure Analysis of 14-syn .......................... 66

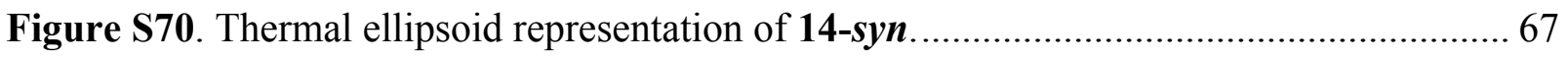

Figure S71. Planes defined to determine the angle at which the mean plane of the pyrrole is angled with respect to the mean plane of the remaining $\mathrm{sp}^{2}$-hybridized heavy atoms of the macrocycle. ....................................................................... 68

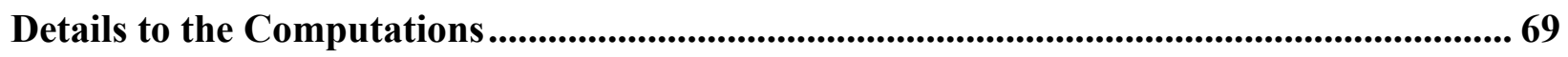

Table S1. Energies of Optimized Geometries of Bacteriochlorins .................................... 69

Optimized Geometries/Coordinates of Compounds Indicated ......................................... 70

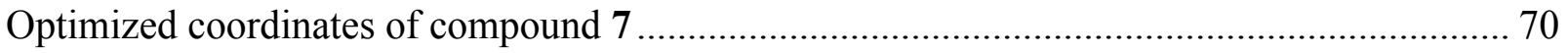

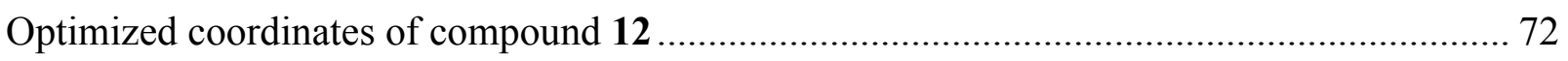

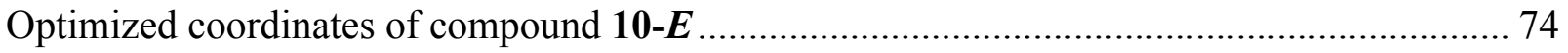

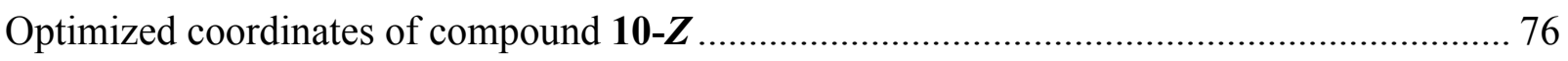

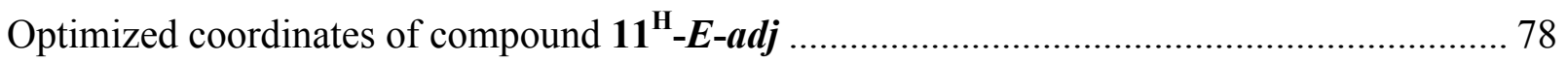

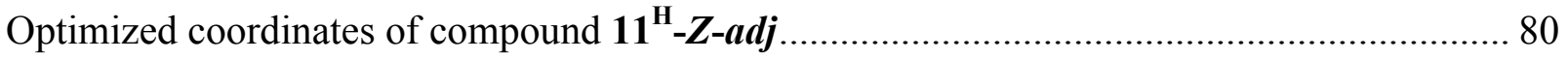

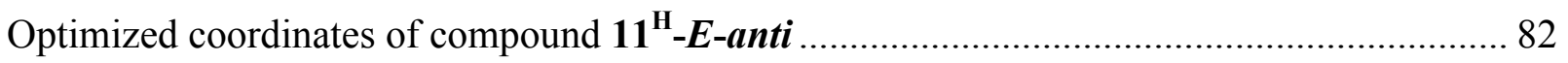

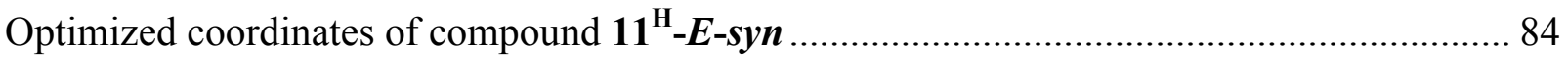

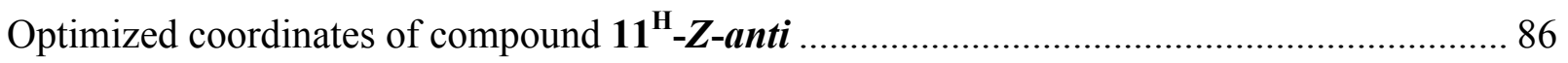

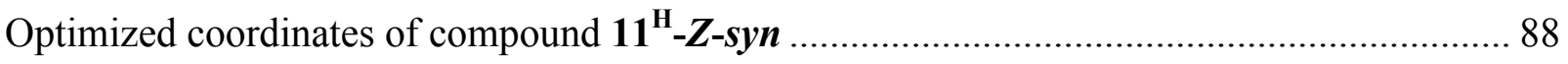




\section{Optical Spectra of the Compounds Prepared}

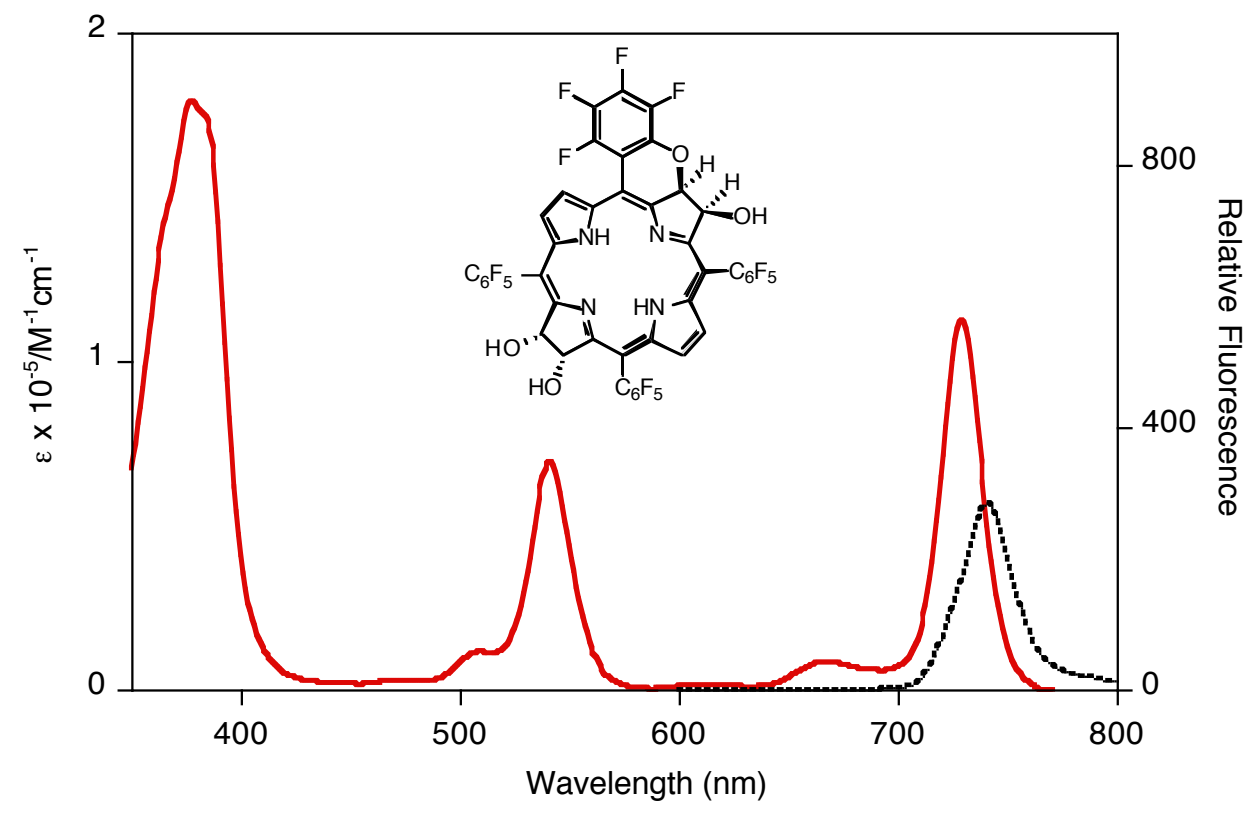

Figure S1. UV-vis absortion (solid red trace) and fluorescence emission (broken black trace) spectra of 10-E $\left(\mathrm{CH}_{2} \mathrm{Cl}_{2}\right)$.

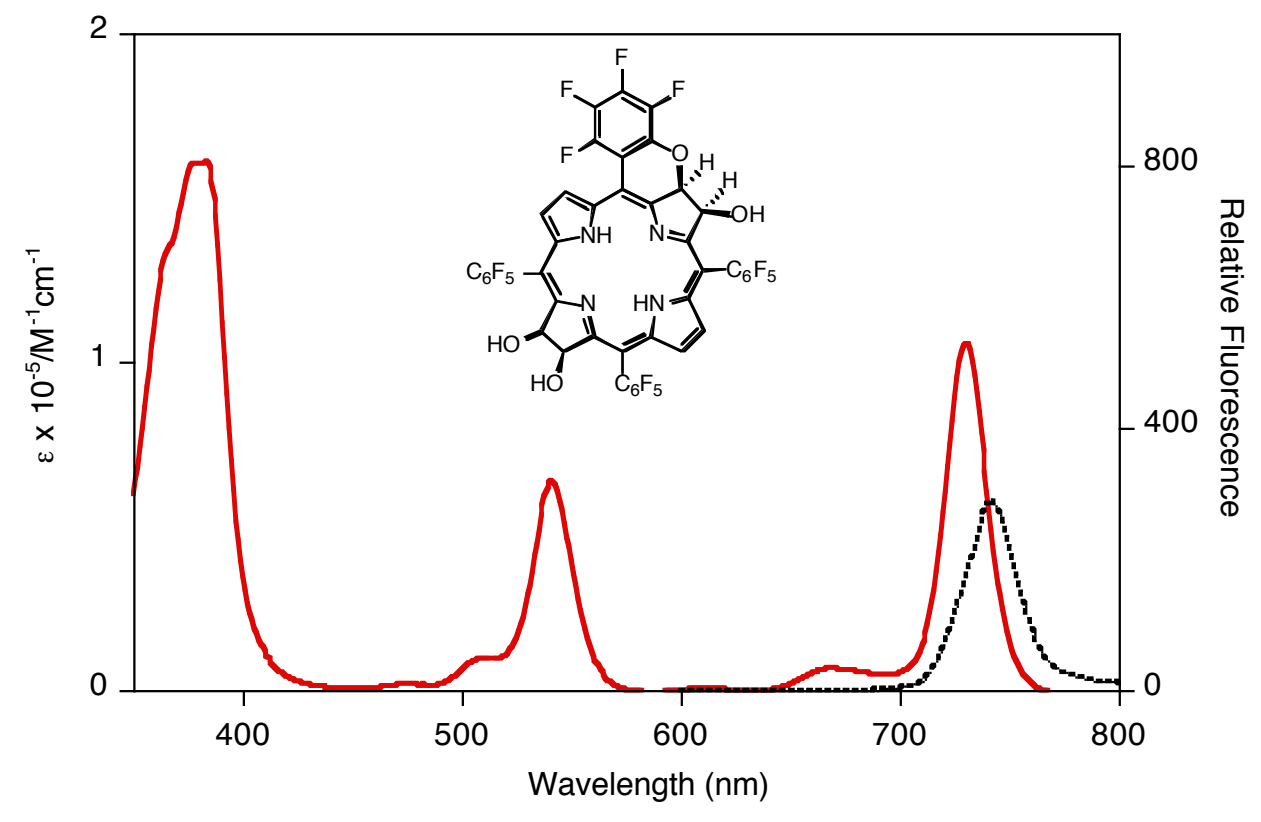

Figure S2. UV-vis absortion (solid red trace) and fluorescence emission (broken black trace) spectra of $10-Z\left(\mathrm{CH}_{2} \mathrm{Cl}_{2}\right)$. 


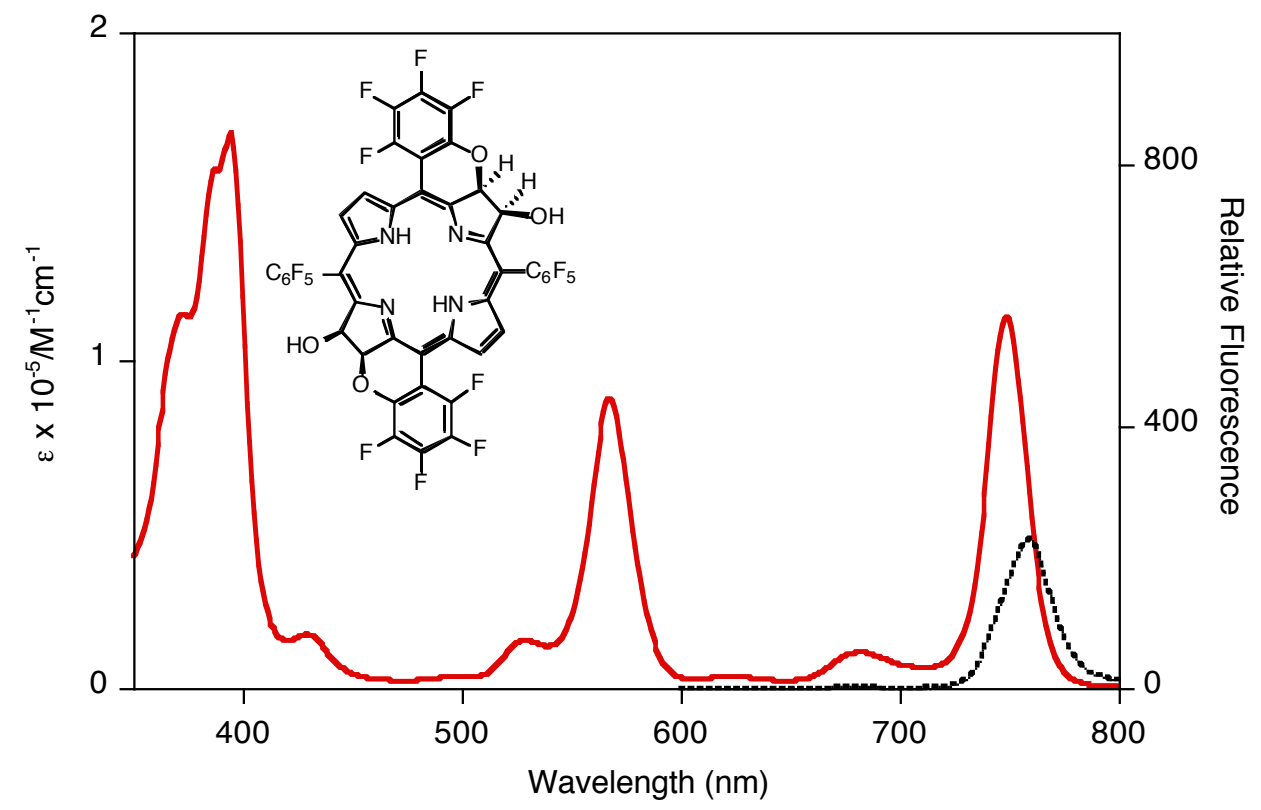

Figure S3. UV-vis absortion (solid red trace) and fluorescence emission (broken black trace) spectra of $11^{\mathrm{H}}-\mathrm{Z}$-anti $\left(\mathrm{CH}_{2} \mathrm{Cl}_{2}\right)$.

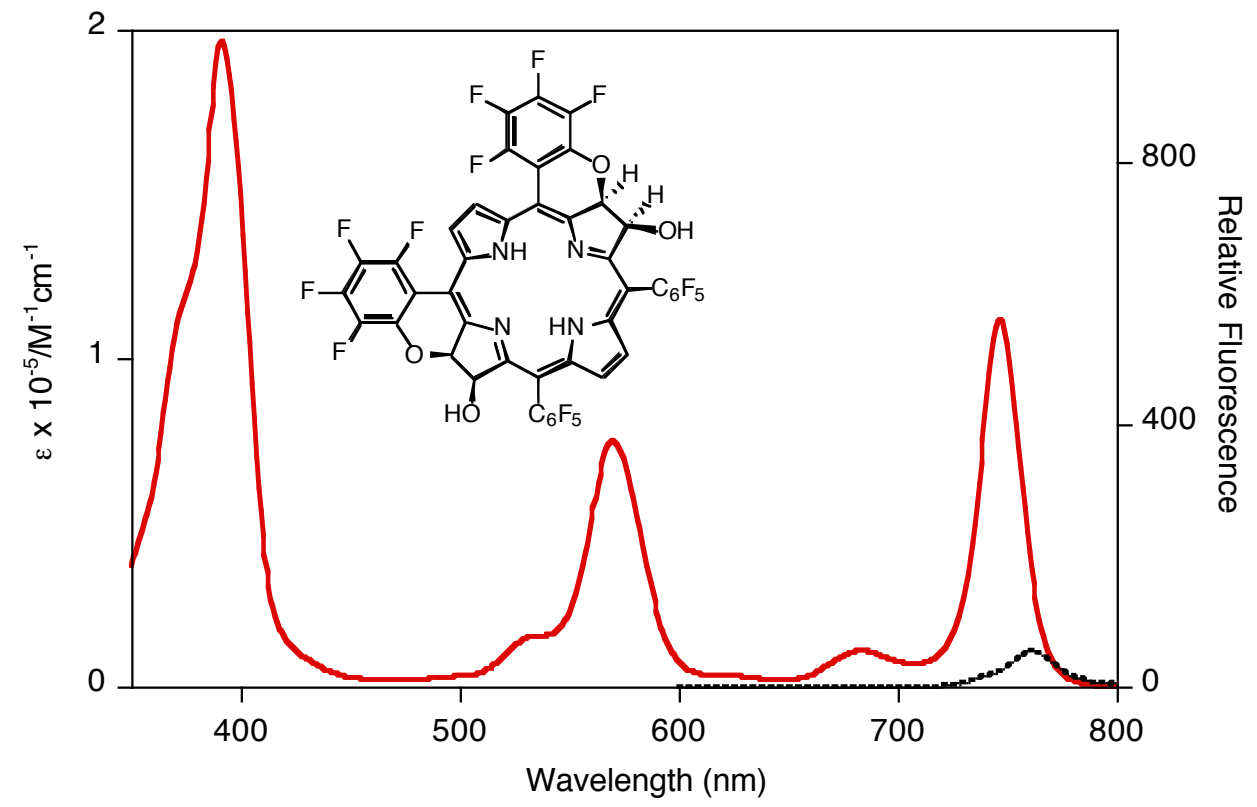

Figure S4. UV-vis absortion (solid red trace) and fluorescence emission (broken black trace) spectra of $11^{\mathrm{H}}-\mathbf{Z}$-syn $\left(\mathrm{CH}_{2} \mathrm{Cl}_{2}\right)$. 


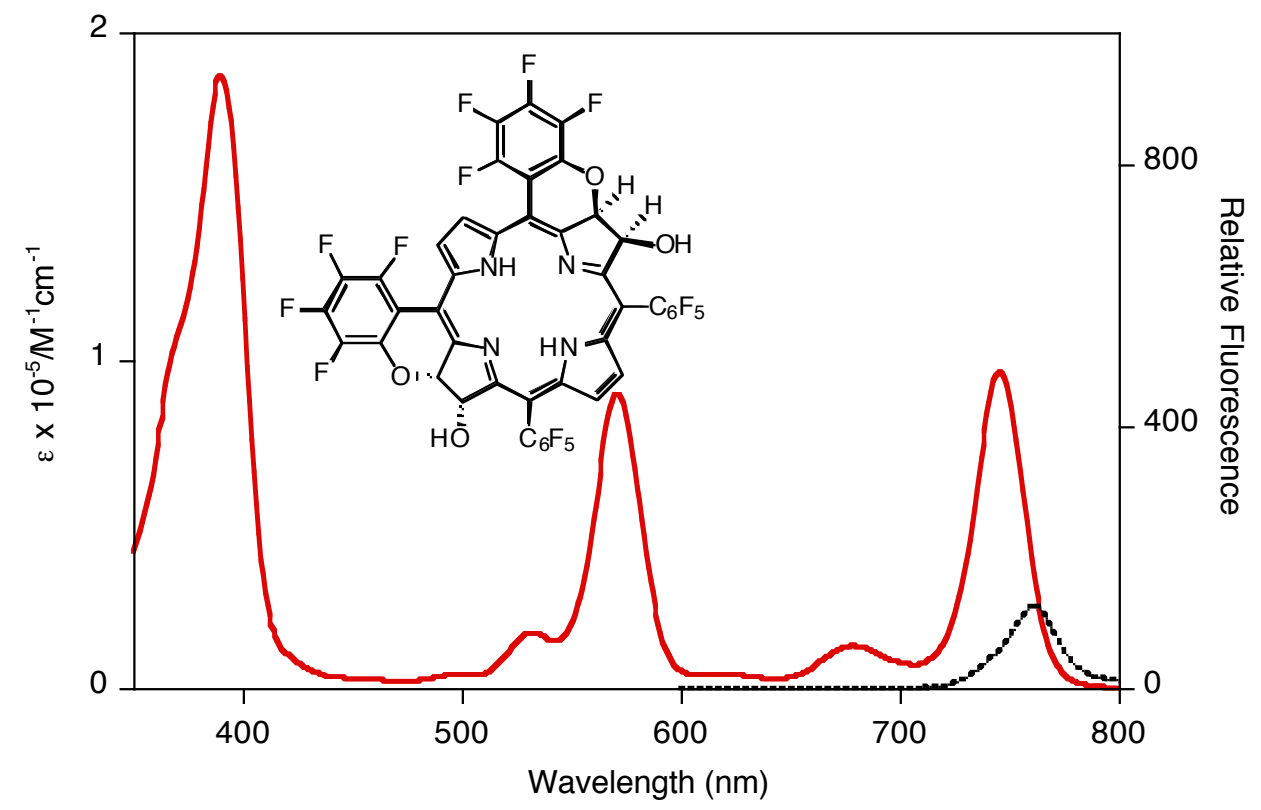

Figure S5. UV-vis absortion (solid red trace) and fluorescence emission (broken black trace) spectra of $11^{\mathrm{H}}-\boldsymbol{E}$-syn $\left(\mathrm{CH}_{2} \mathrm{Cl}_{2}\right)$.

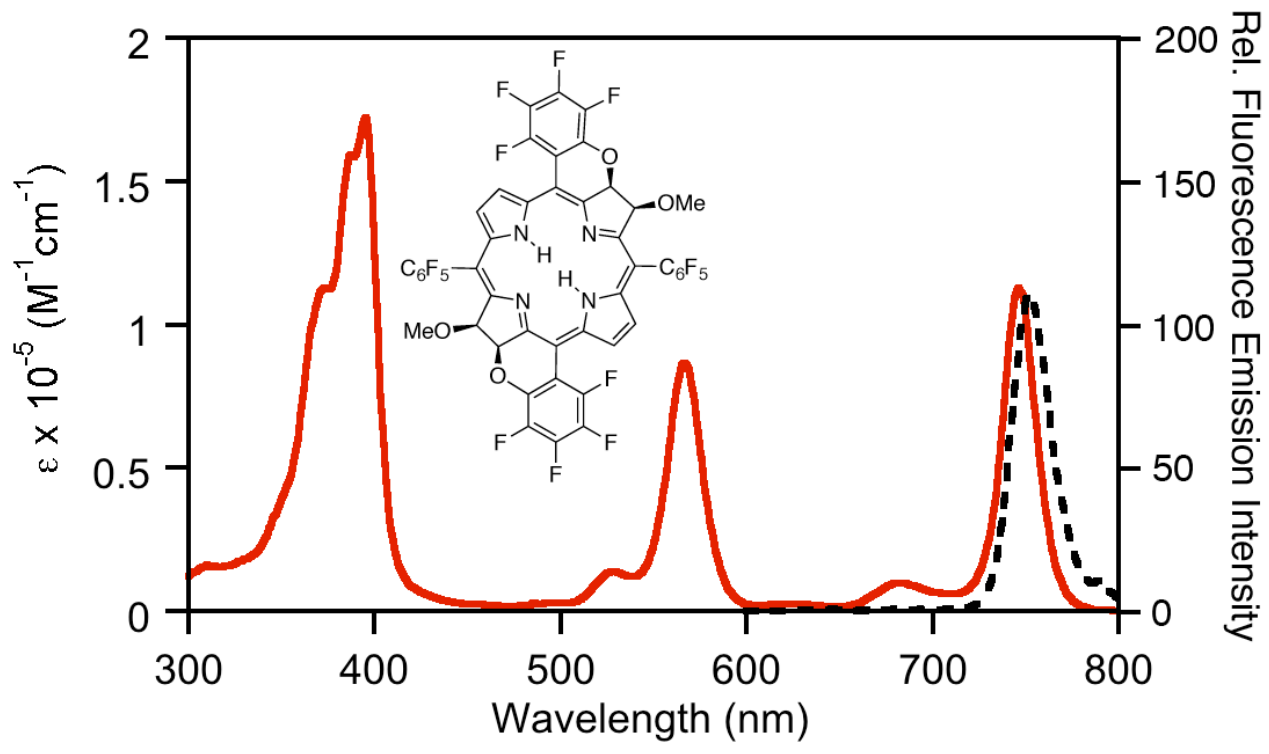

Figure S6. UV-vis absortion (solid red trace) and fluorescence emission (broken black trace) spectra of $11^{\mathrm{Me}}$-Z-anti $\left(\mathrm{CH}_{2} \mathrm{Cl}_{2}\right)$. 


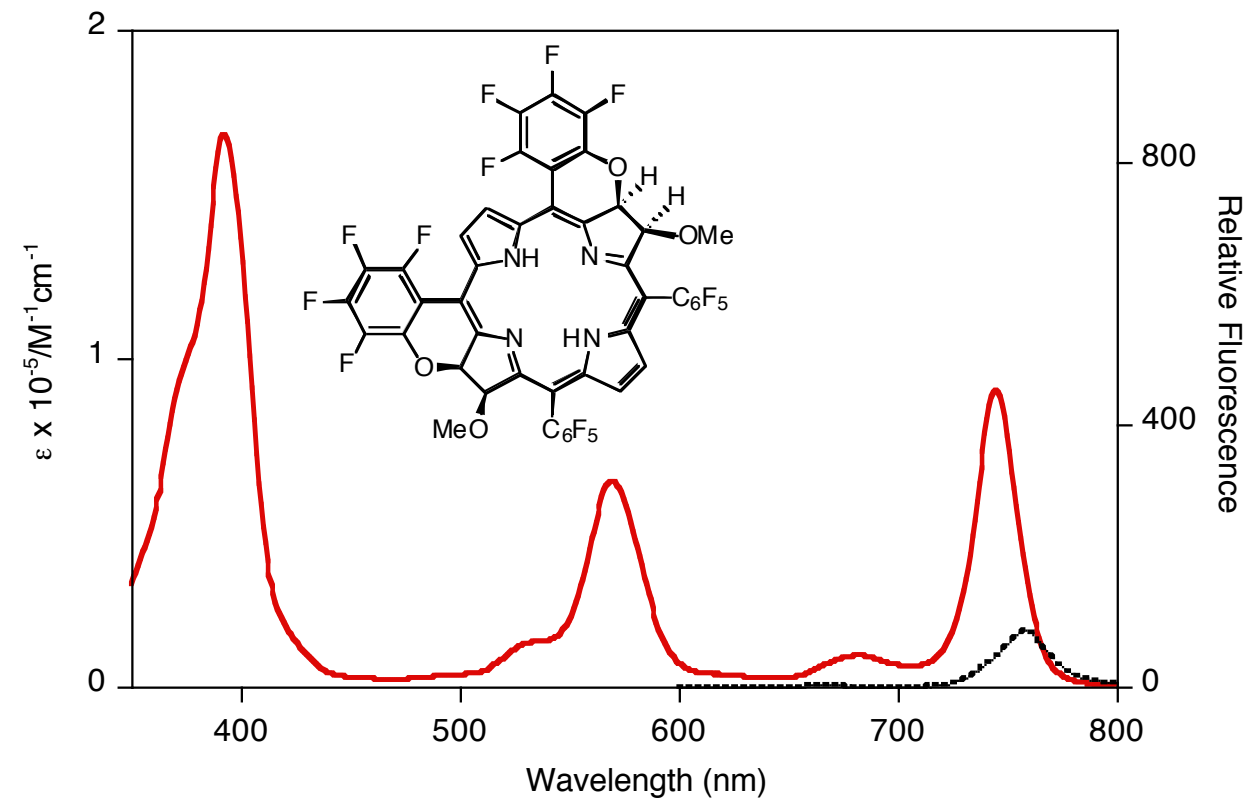

Figure S7. UV-vis absortion (solid red trace) and fluorescence emission (broken black trace) spectra of $11^{\mathrm{Me}}-\mathrm{Z}-\mathrm{syn}\left(\mathrm{CH}_{2} \mathrm{Cl}_{2}\right)$.

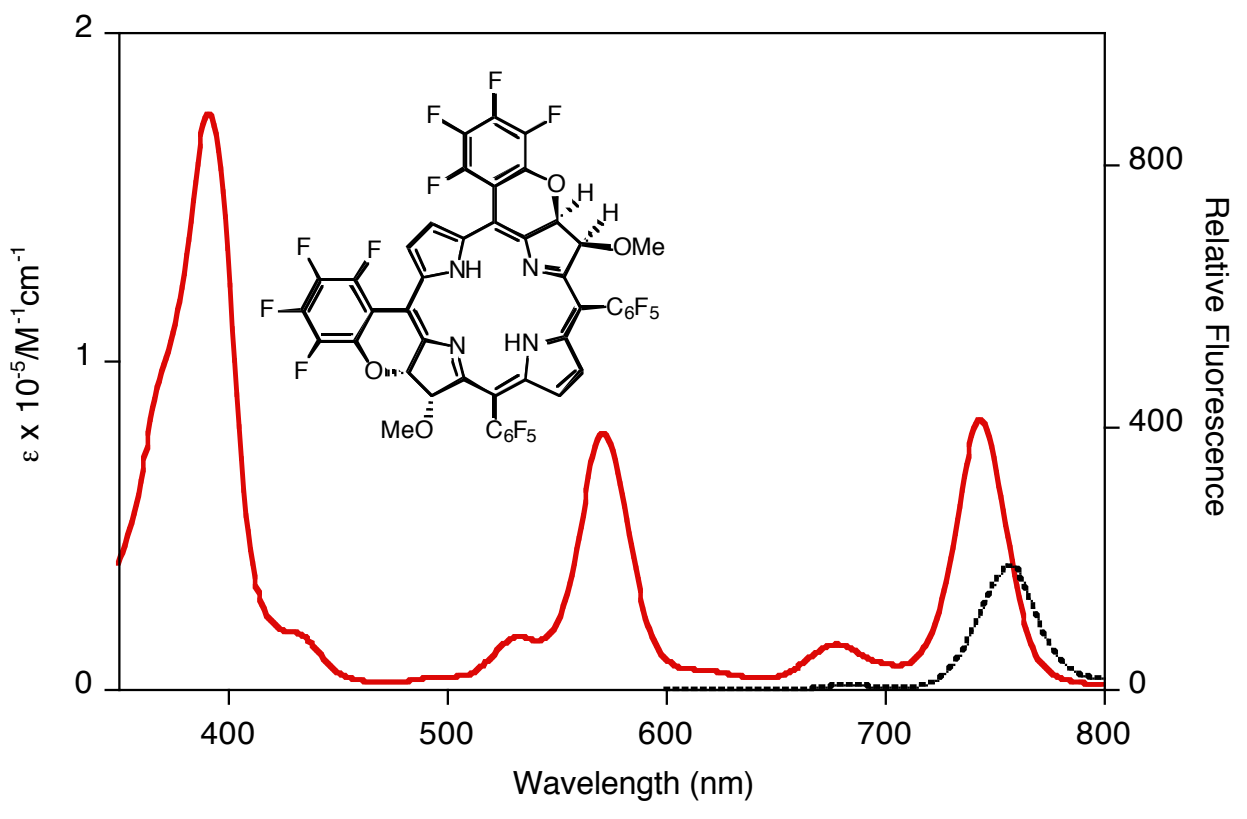

Figure S8. UV-vis absortion (solid red trace) and fluorescence emission (broken black trace) spectra of $11^{\mathrm{Me}}-\boldsymbol{E}$-syn $\left(\mathrm{CH}_{2} \mathrm{Cl}_{2}\right)$. 


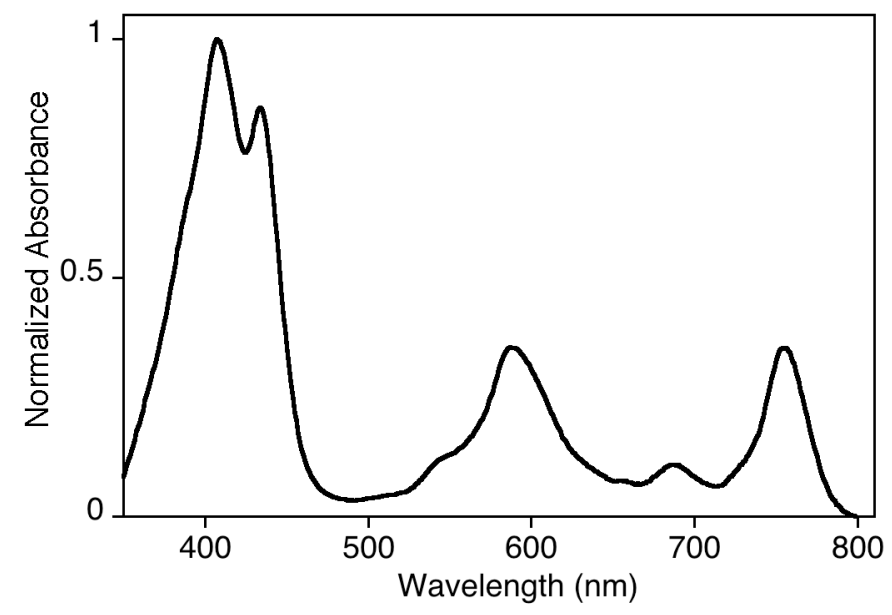

Figure S9. UV-vis spectrum of $13-Z\left(\mathrm{CH}_{2} \mathrm{Cl}_{2}\right)$.

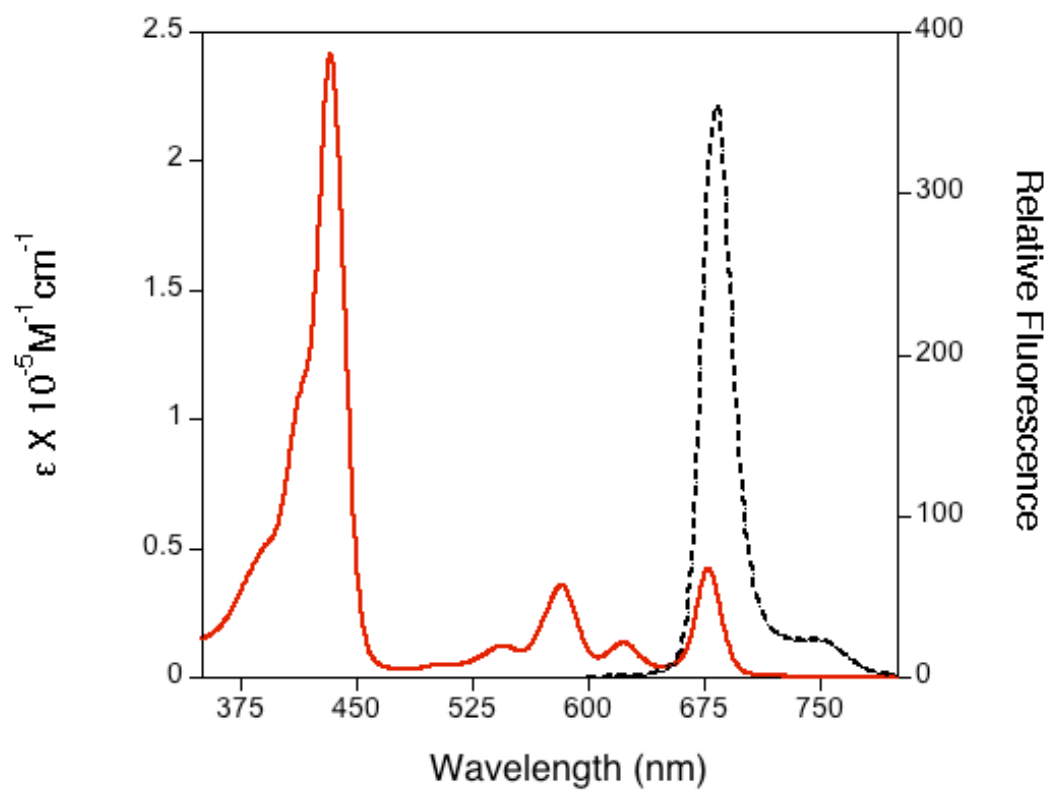

Figure S10. UV-vis absortion (solid red trace) and fluorescence emission (broken black trace) spectra of 14-syn $\left(\mathrm{CH}_{2} \mathrm{Cl}_{2}\right)$ 


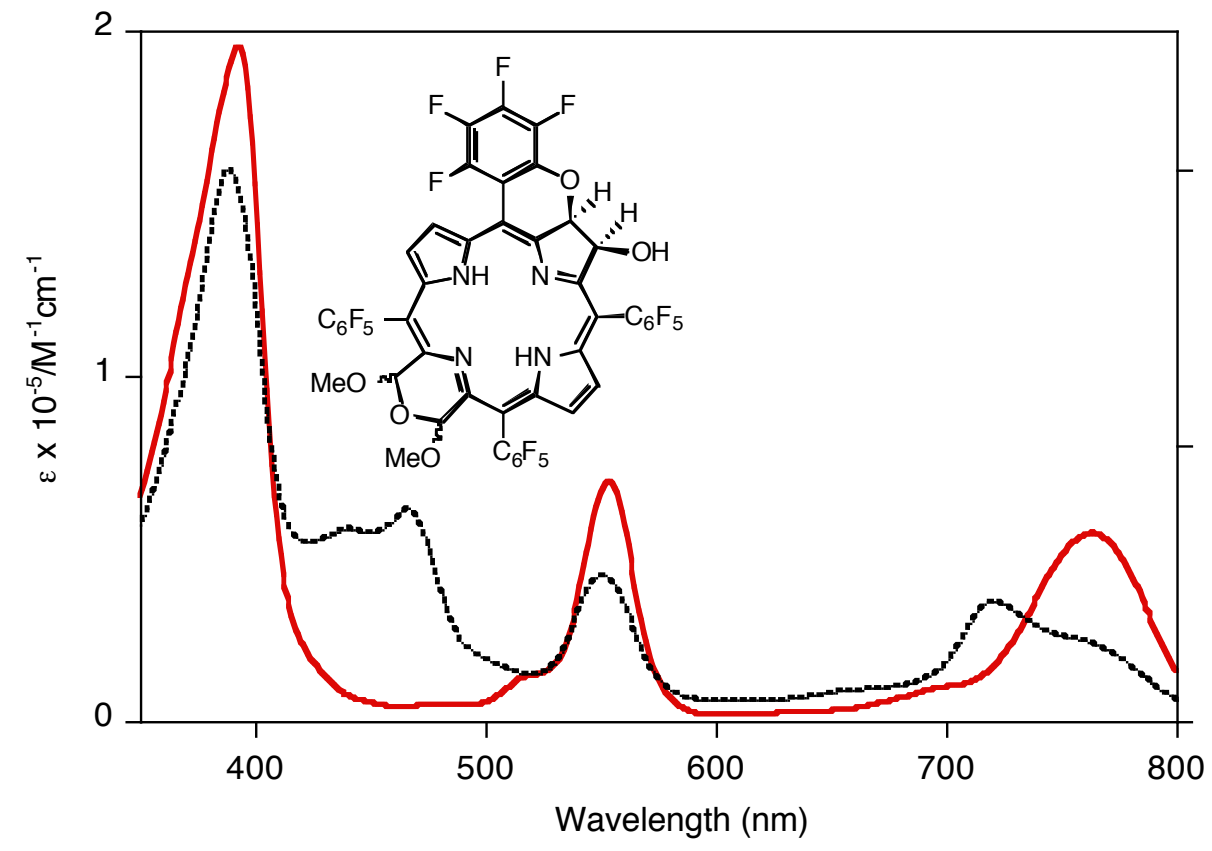

Figure S11. UV-vis spectra of $14^{\mathrm{Me}}$ in neutral $\left(\mathrm{CH}_{2} \mathrm{Cl}_{2}\right.$; solid red trace $)$ and acidified $\left(\mathrm{CH}_{2} \mathrm{Cl}_{2}\right.$ with $\sim 0.1 \%$ of TFA; broken black trace) media.

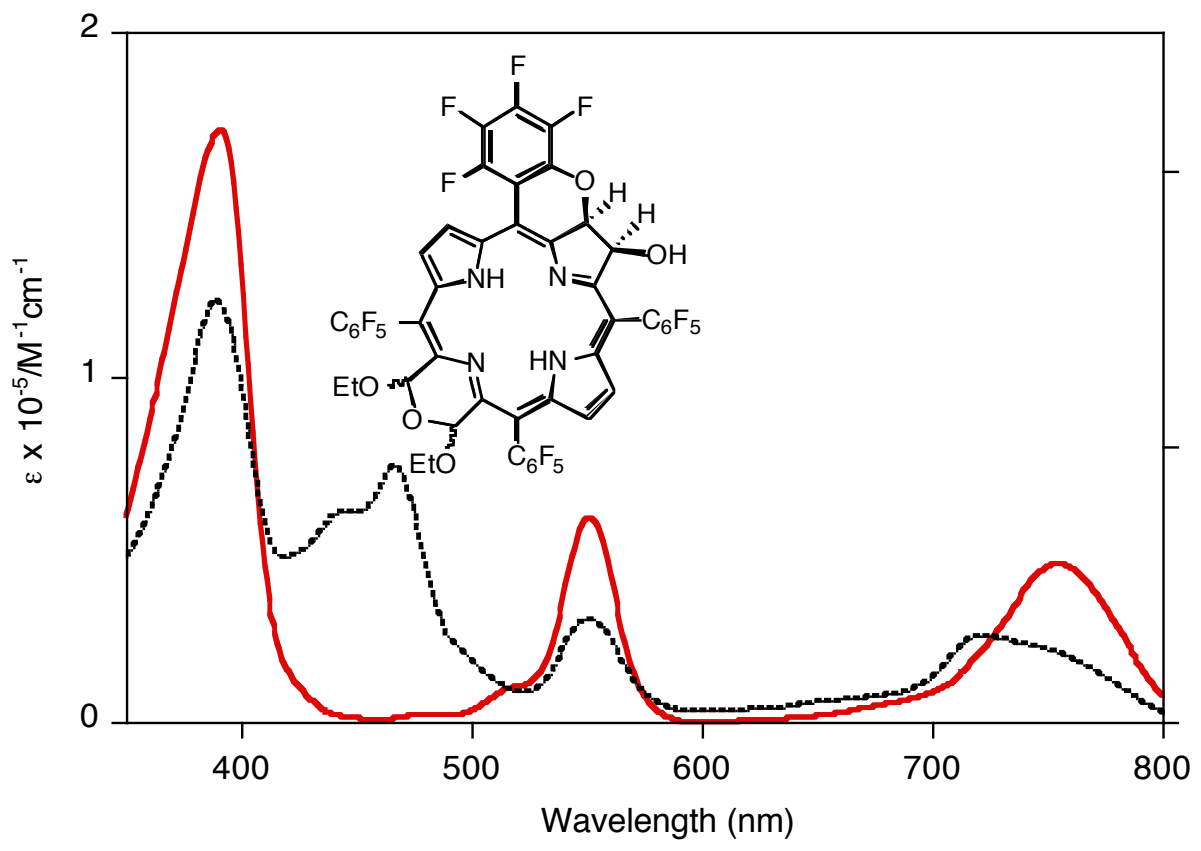

Figure S12. UV-vis spectra of $14^{\mathrm{Et}}$ in neutral $\left(\mathrm{CH}_{2} \mathrm{Cl}_{2}\right.$; solid red trace) and acidified $\left(\mathrm{CH}_{2} \mathrm{Cl}_{2}\right.$ with $\sim 0.1 \%$ of TFA; broken black trace) media. 


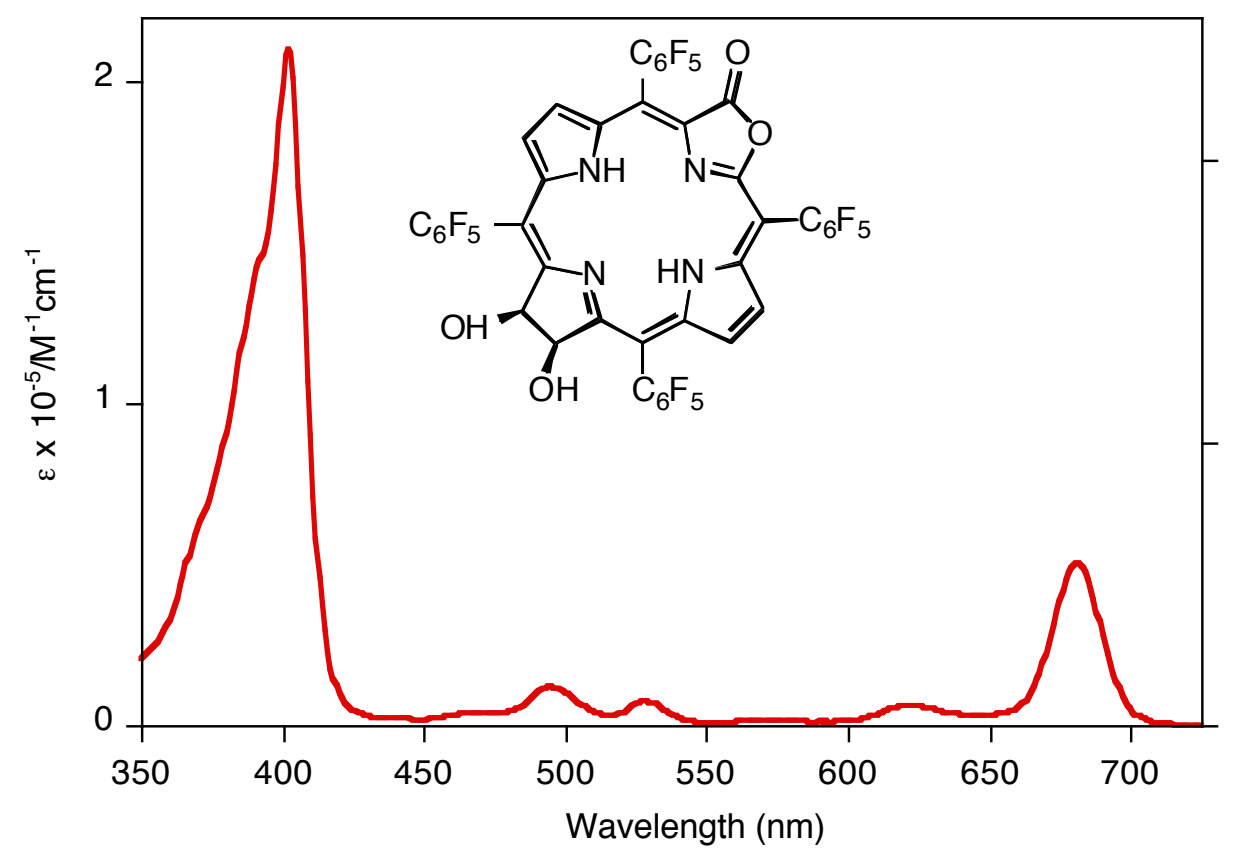

Figure S13. UV-vis absortion spectrum of 17.

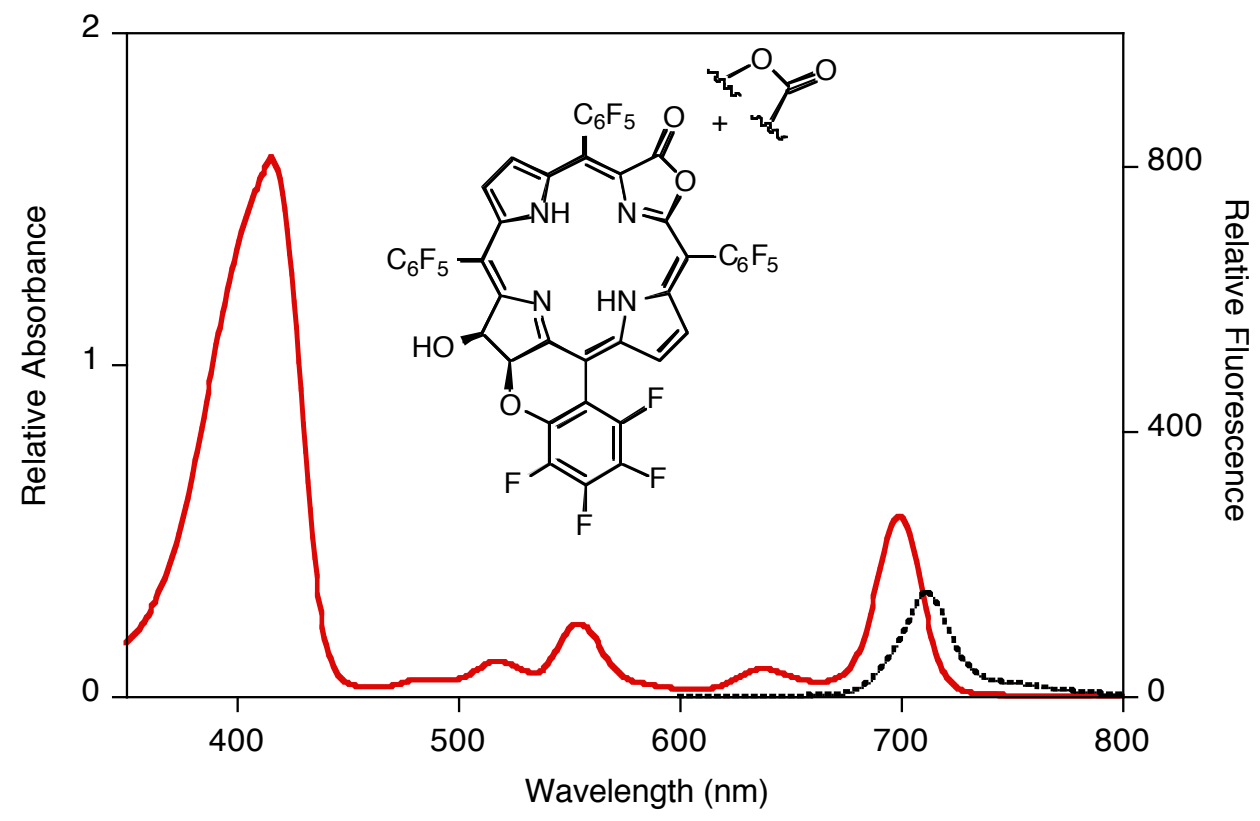

Figure S14. UV-vis absortion (solid red trace) and fluorescence emission (broken black trace) spectra of $16\left(\mathrm{CH}_{2} \mathrm{Cl}_{2}\right)$. 


\section{${ }^{1} \mathrm{H},{ }^{13} \mathrm{C}$, and ${ }^{19} \mathrm{~F}$ NMR Spectra of the Compounds Described}

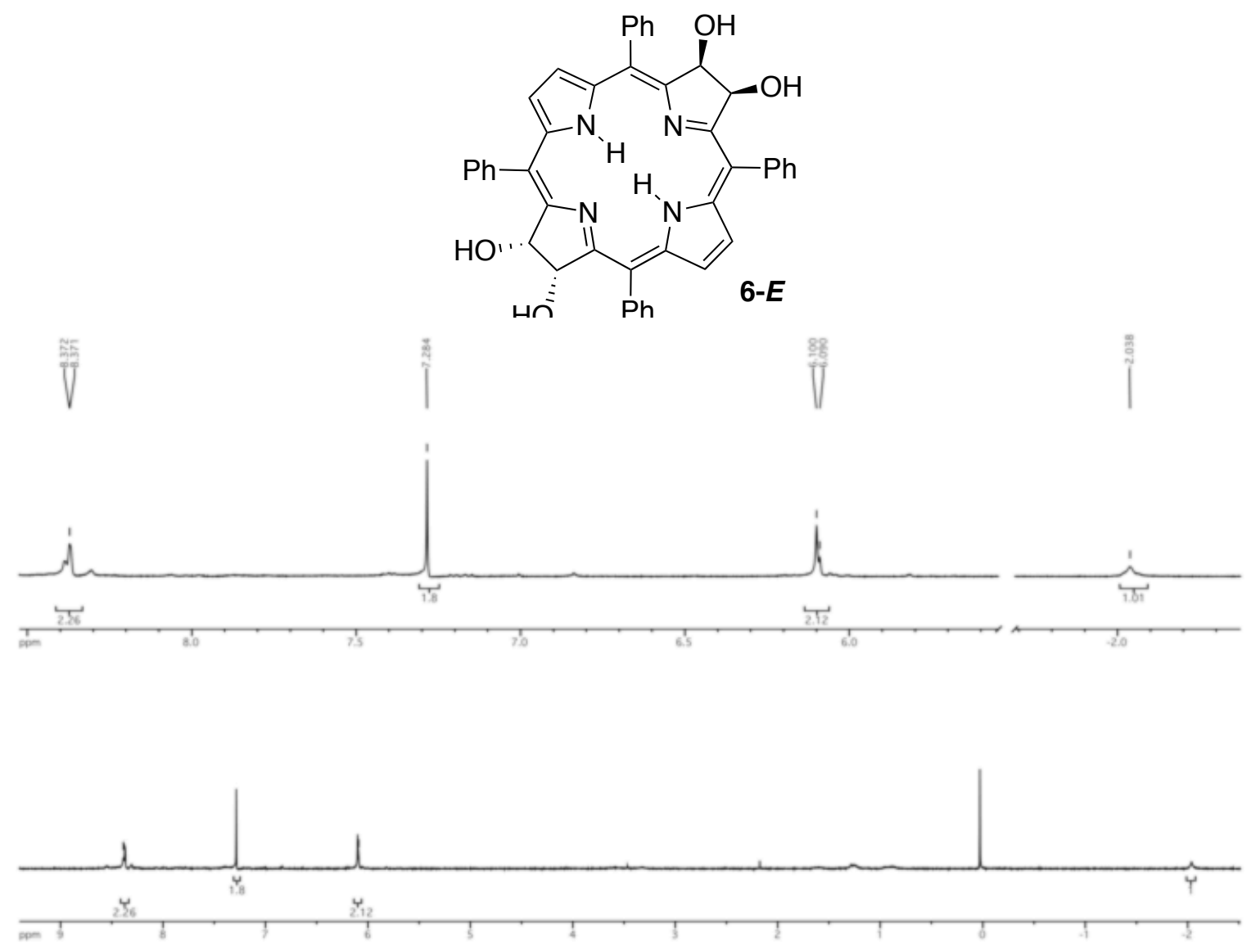

Figure S15. ${ }^{1} \mathrm{H}$ NMR spectrum $\left(400 \mathrm{MHz}, \mathrm{CDCl}_{3}\right)$ of $\mathbf{6 - \boldsymbol { E }}$.
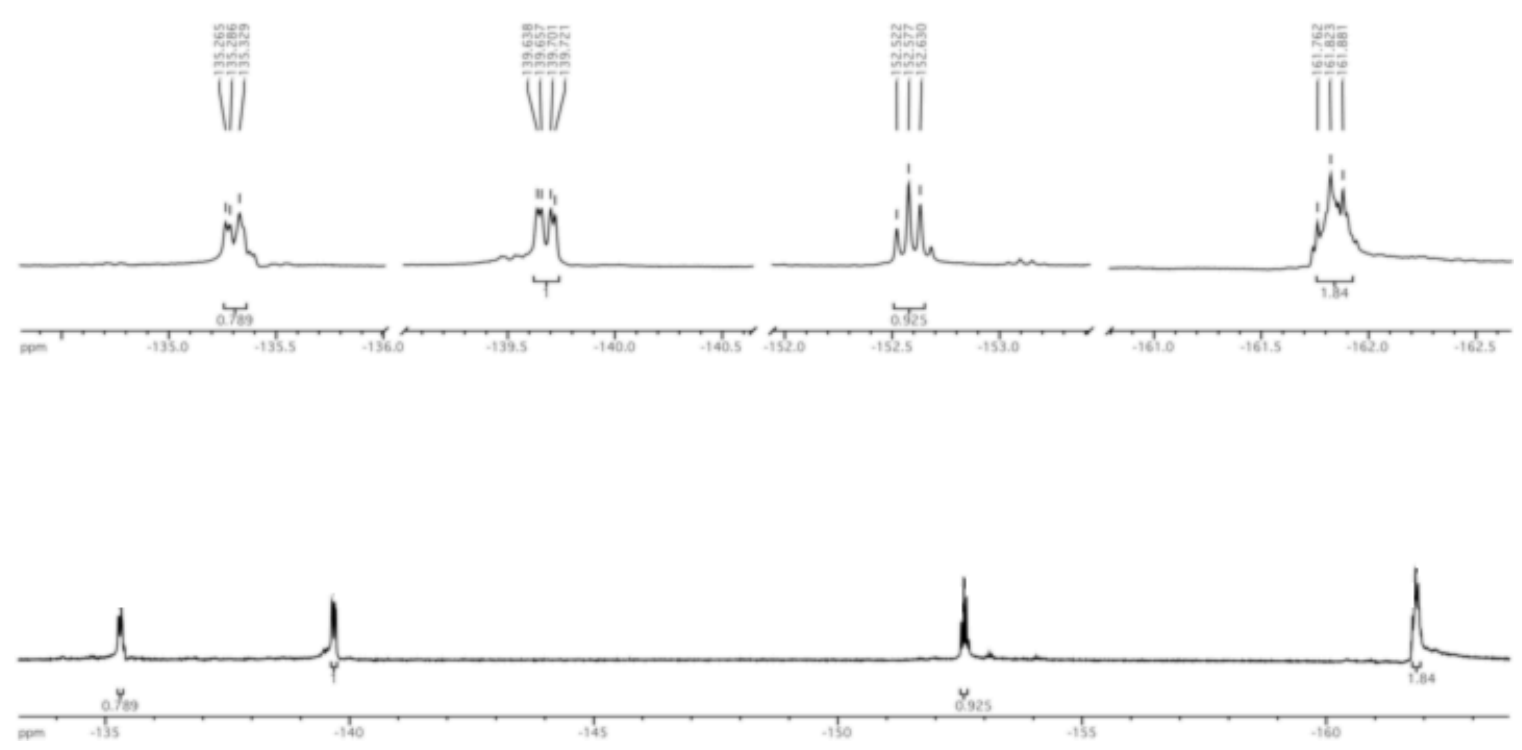

Figure S16. ${ }^{19} \mathrm{~F}$ NMR spectrum $\left(376 \mathrm{MHz}, \mathrm{CDCl}_{3}\right)$ of $\mathbf{6}-\boldsymbol{E}$. 


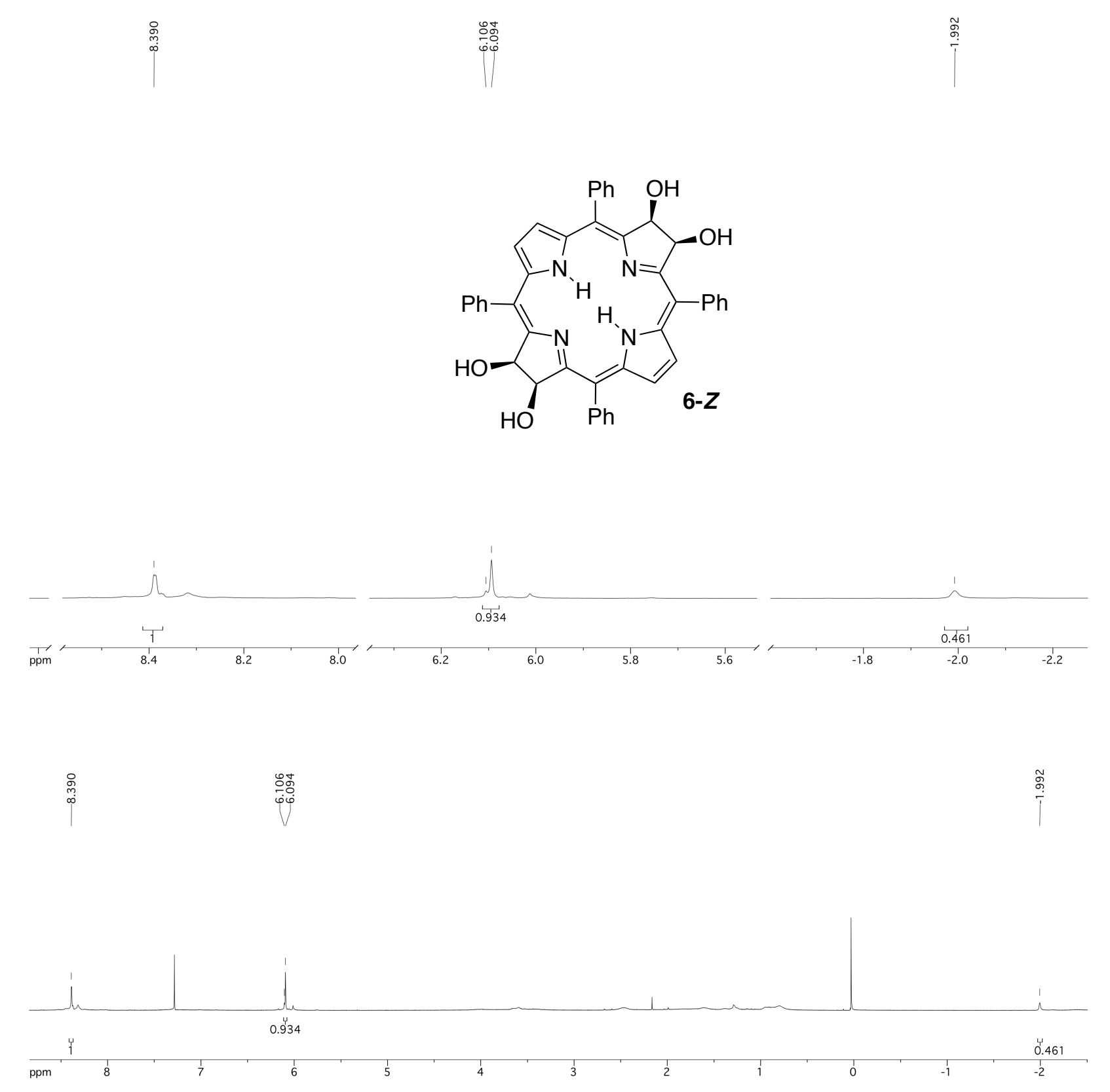

Figure S17. ${ }^{1} \mathrm{H}$ NMR spectrum $\left(400 \mathrm{MHz}, \mathrm{CDCl}_{3}\right)$ of $\mathbf{6 - Z}$. 


\begin{tabular}{|c|c|c|c|}
\hline 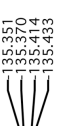 & 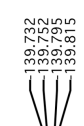 & 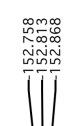 & 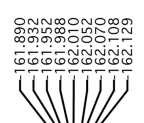 \\
\hline
\end{tabular}

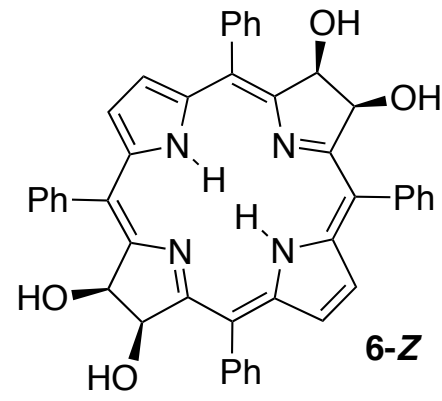
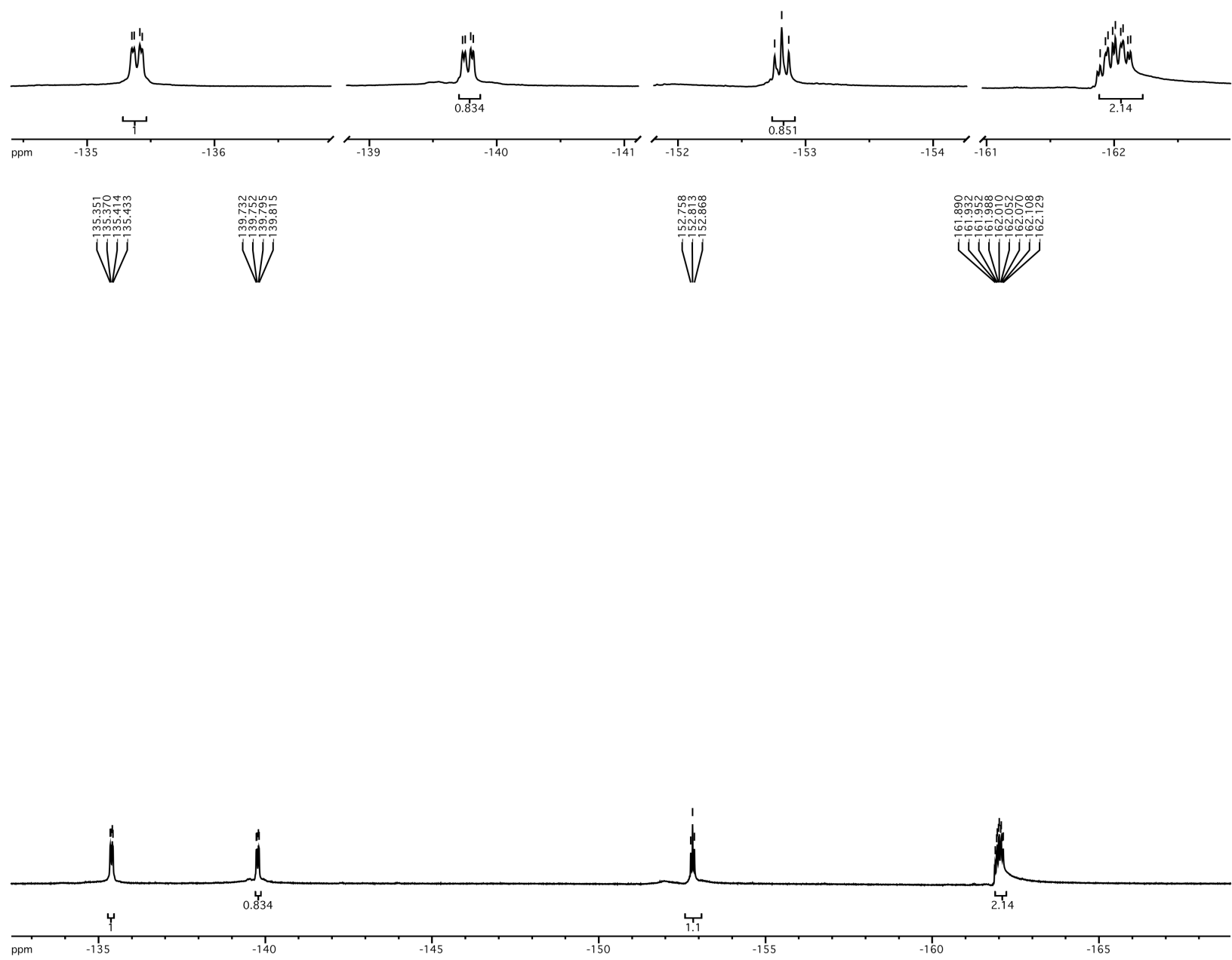

Figure S18. ${ }^{19} \mathrm{~F}$ NMR spectrum $\left(376 \mathrm{MHz}, \mathrm{CDCl}_{3}\right)$ of $\mathbf{6}-\boldsymbol{Z}$. 

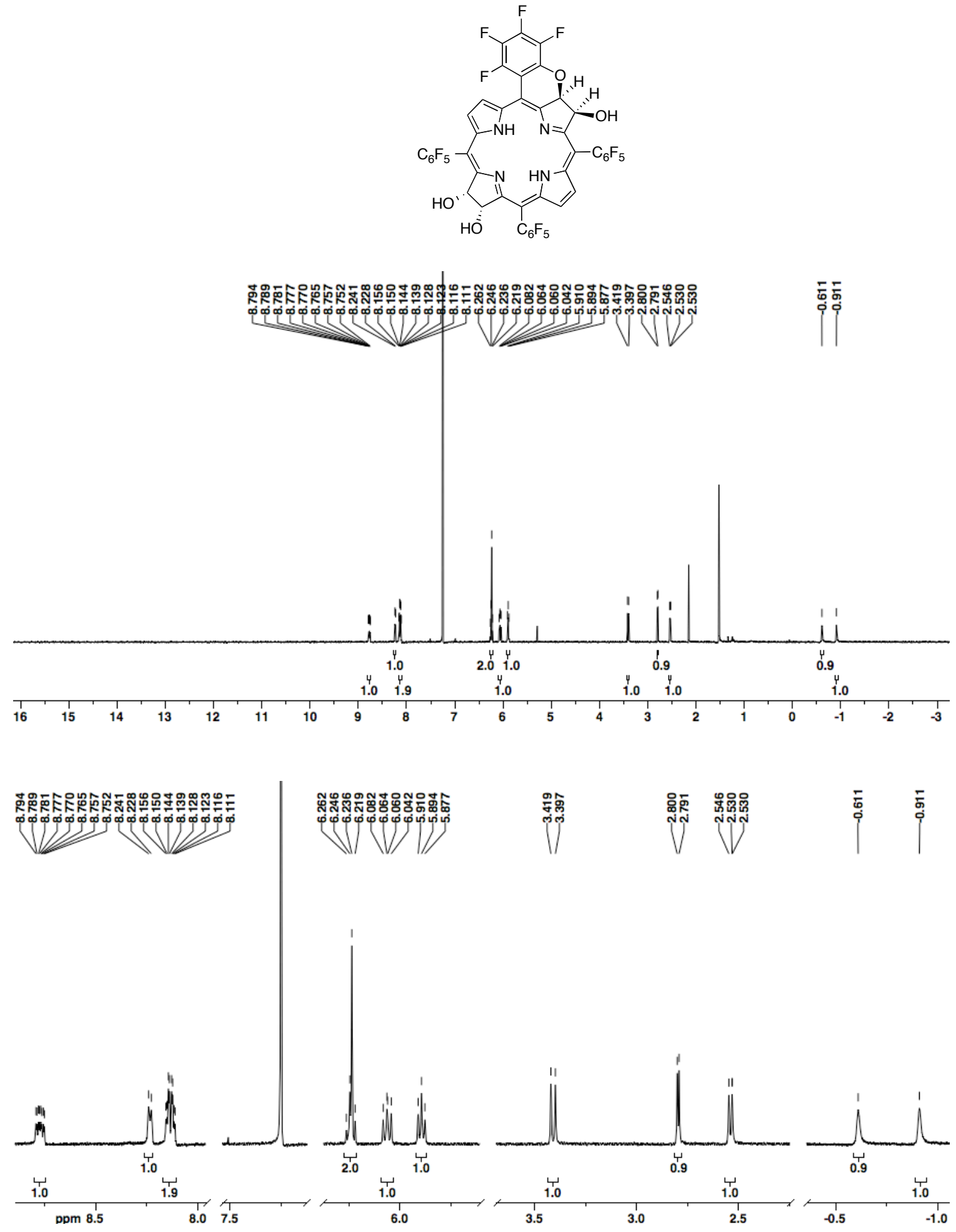

Figure S19. ${ }^{1} \mathrm{H}$ NMR spectrum $\left(400 \mathrm{MHz}, \mathrm{CDCl}_{3}\right)$ of $\mathbf{1 0 -} \boldsymbol{E}$. 


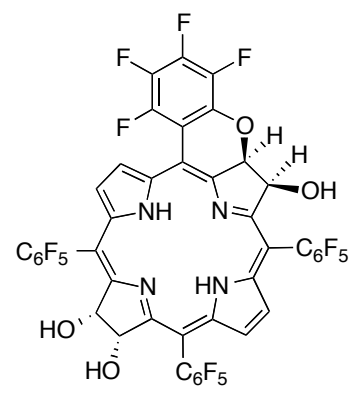
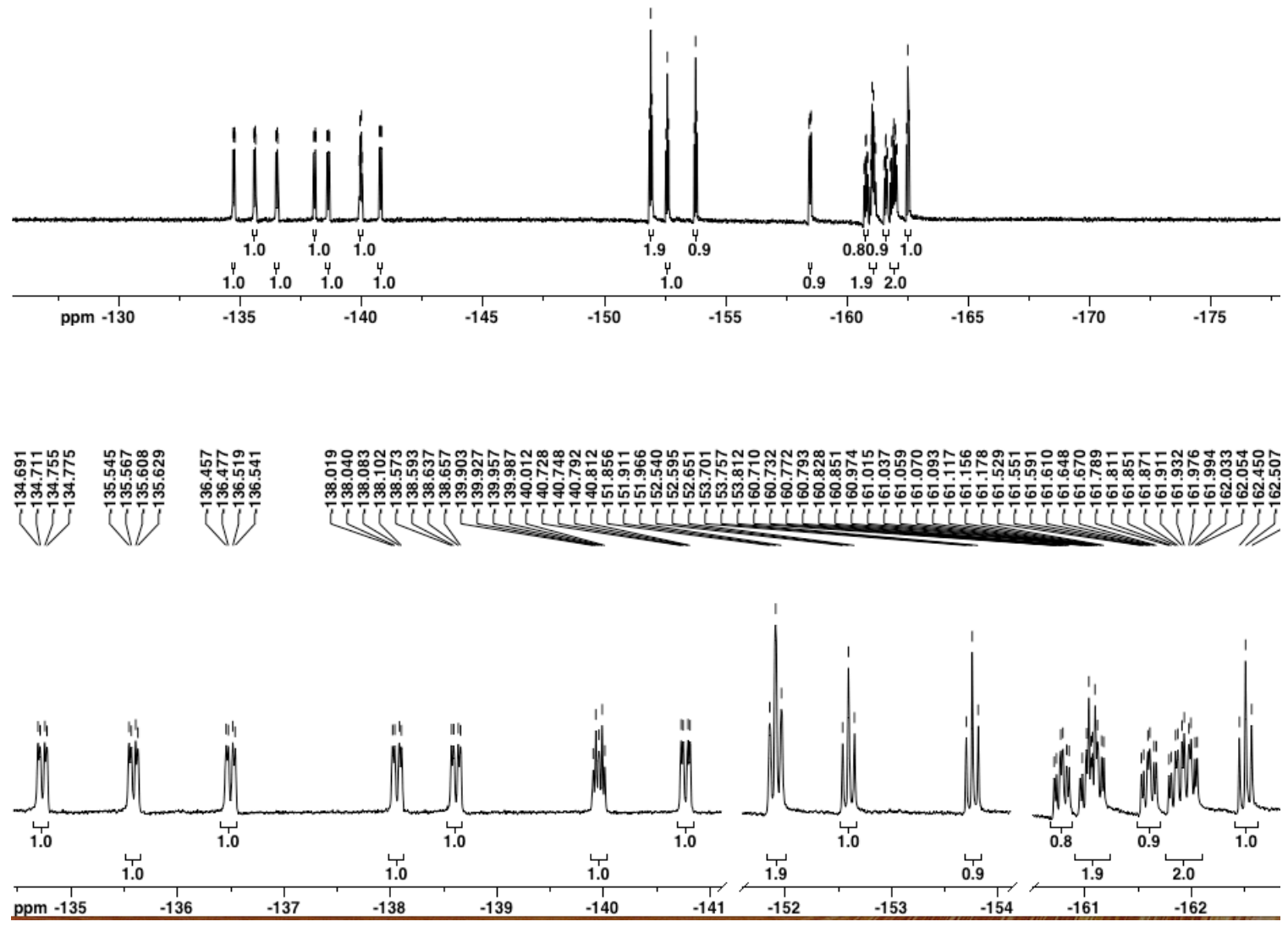

Figure S20. ${ }^{19} \mathrm{~F}$ NMR spectrum $\left(376 \mathrm{MHz}, \mathrm{CDCl}_{3}\right)$ of $\mathbf{1 0}-\boldsymbol{E}$. 

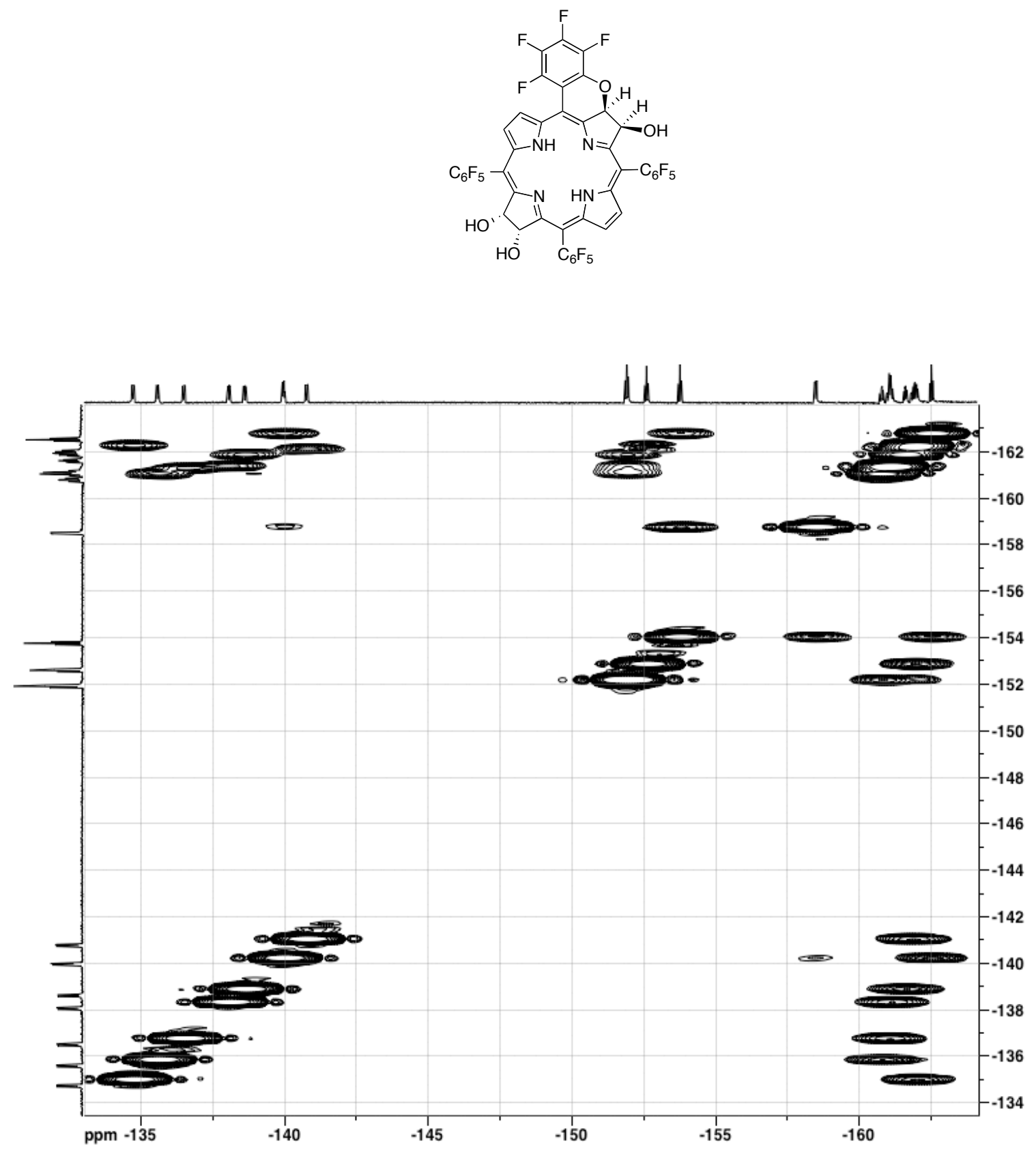

Figure S21. ${ }^{19} \mathrm{~F},{ }^{19} \mathrm{~F}$ QF-COSY NMR spectrum $\left(376 \mathrm{MHz}, \mathrm{CDCl}_{3}\right)$ of $\mathbf{1 0 -} \boldsymbol{E}$. 

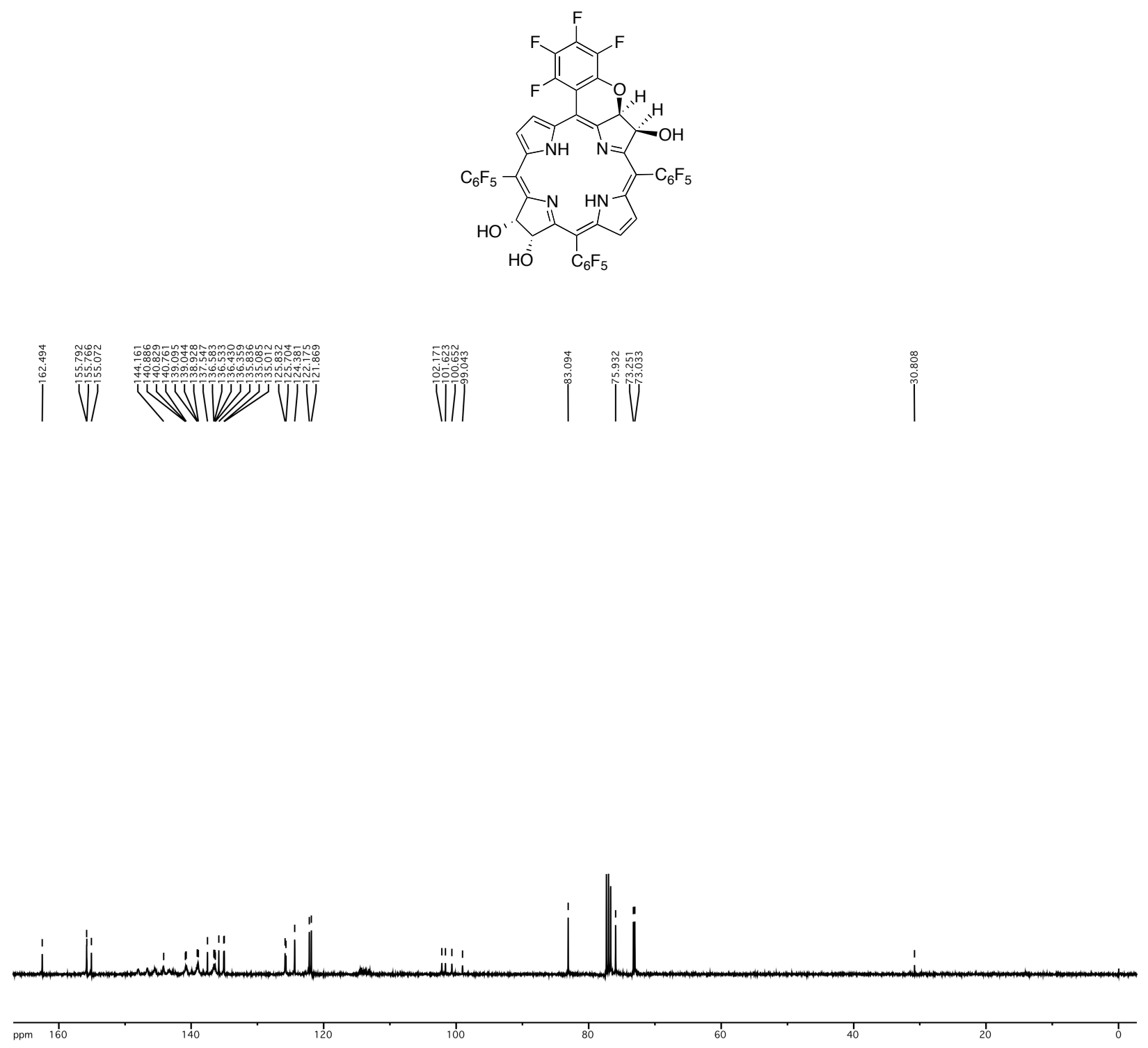

Figure S22. ${ }^{13} \mathrm{C}$ NMR spectrum $\left(100 \mathrm{MHz}, \mathrm{CDCl}_{3}\right)$ of $\mathbf{1 0 - E}$. 

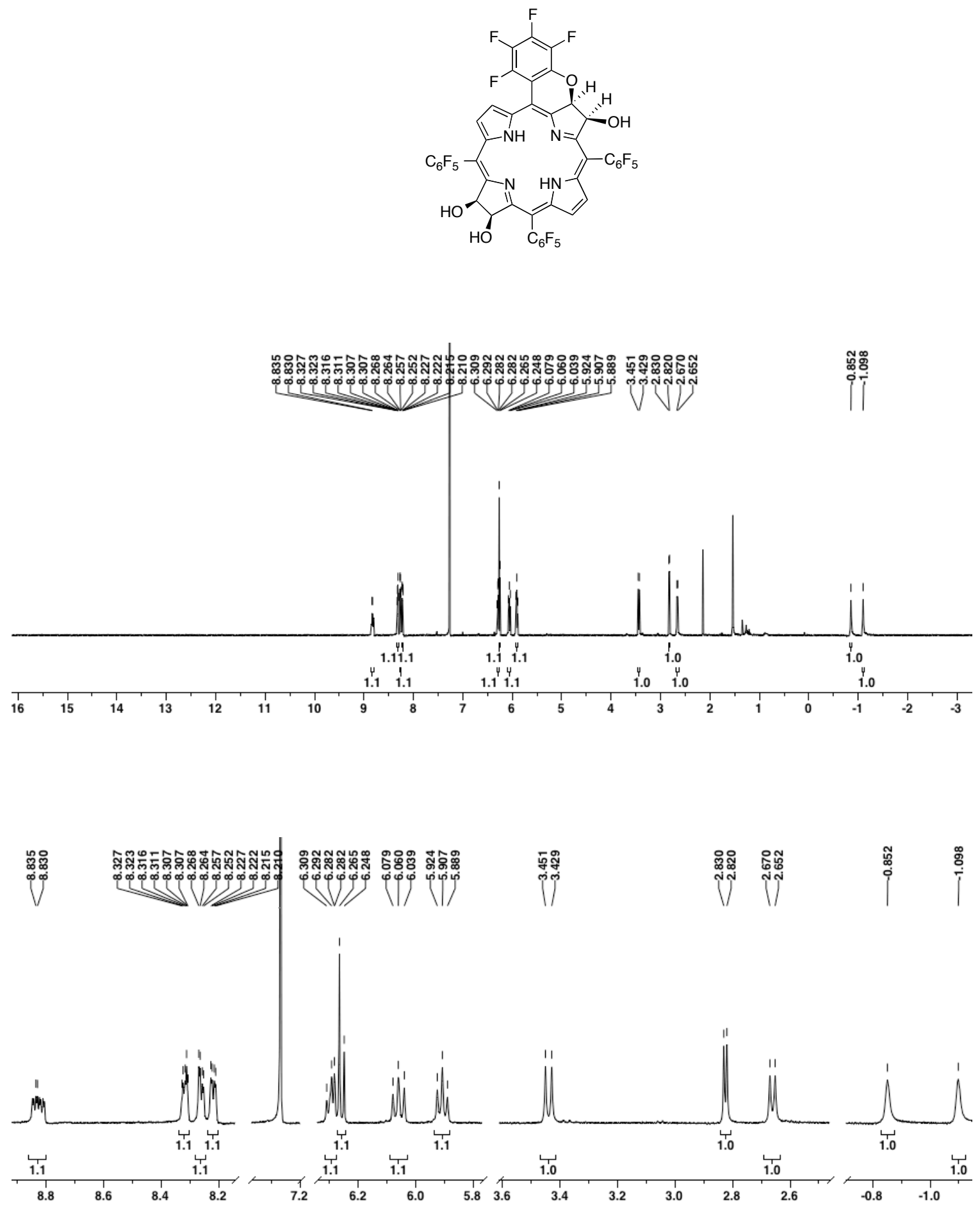

Figure S23. ${ }^{1} \mathrm{H}$ NMR spectrum $\left(400 \mathrm{MHz}, \mathrm{CDCl}_{3}\right.$ ) of $\mathbf{1 0 - Z}$. 


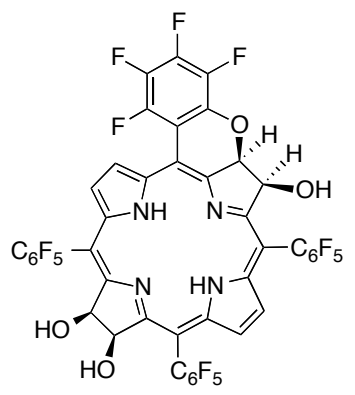

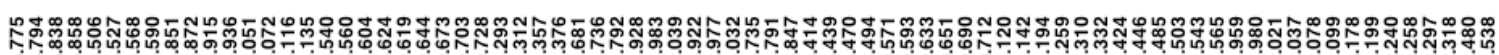

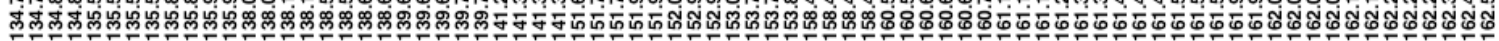

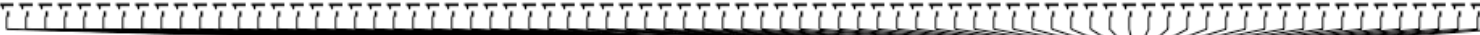
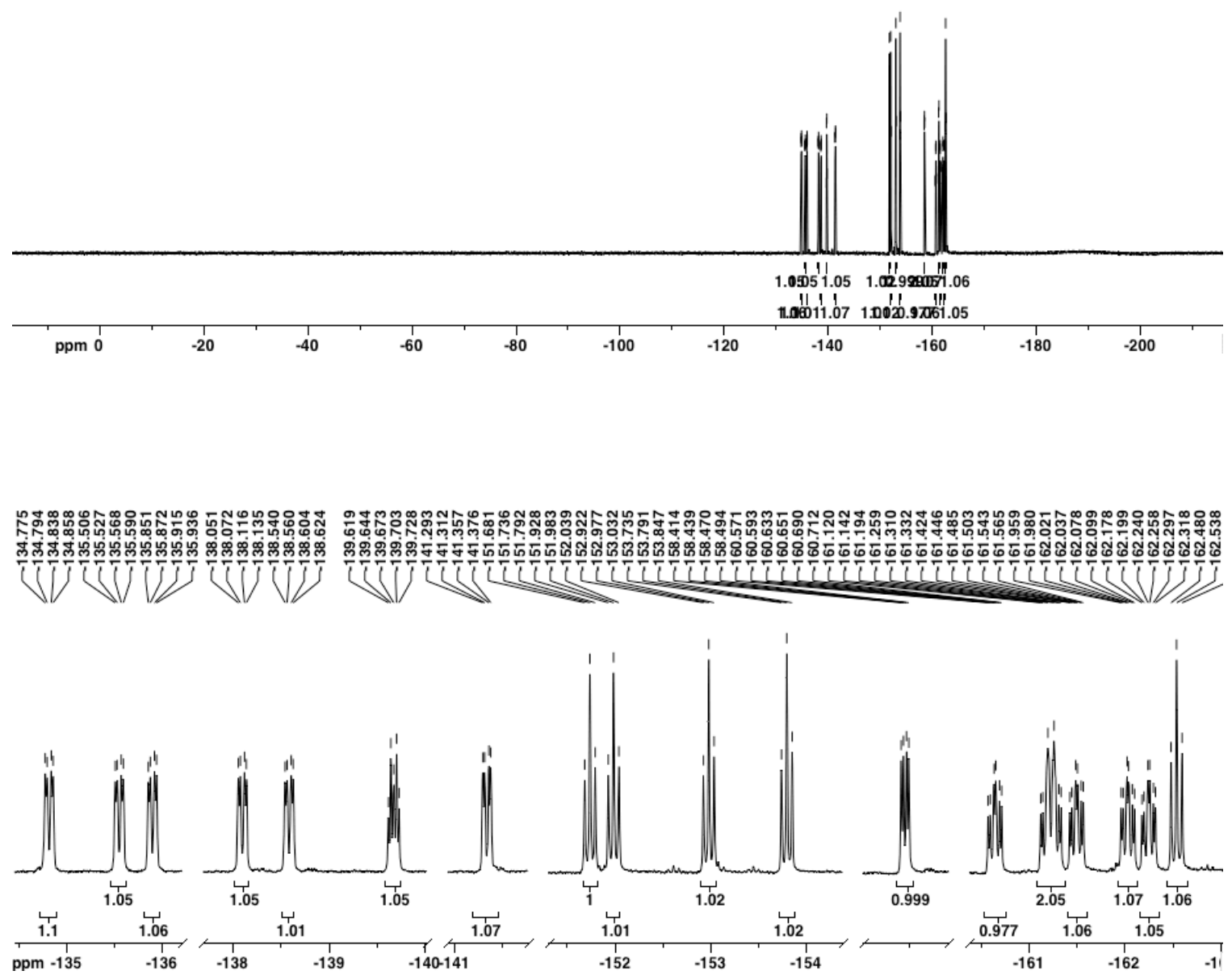

Figure S24. ${ }^{19} \mathrm{~F}$ NMR spectrum $\left(376 \mathrm{MHz}, \mathrm{CDCl}_{3}\right)$ of $\mathbf{1 0}-\boldsymbol{Z}$. 

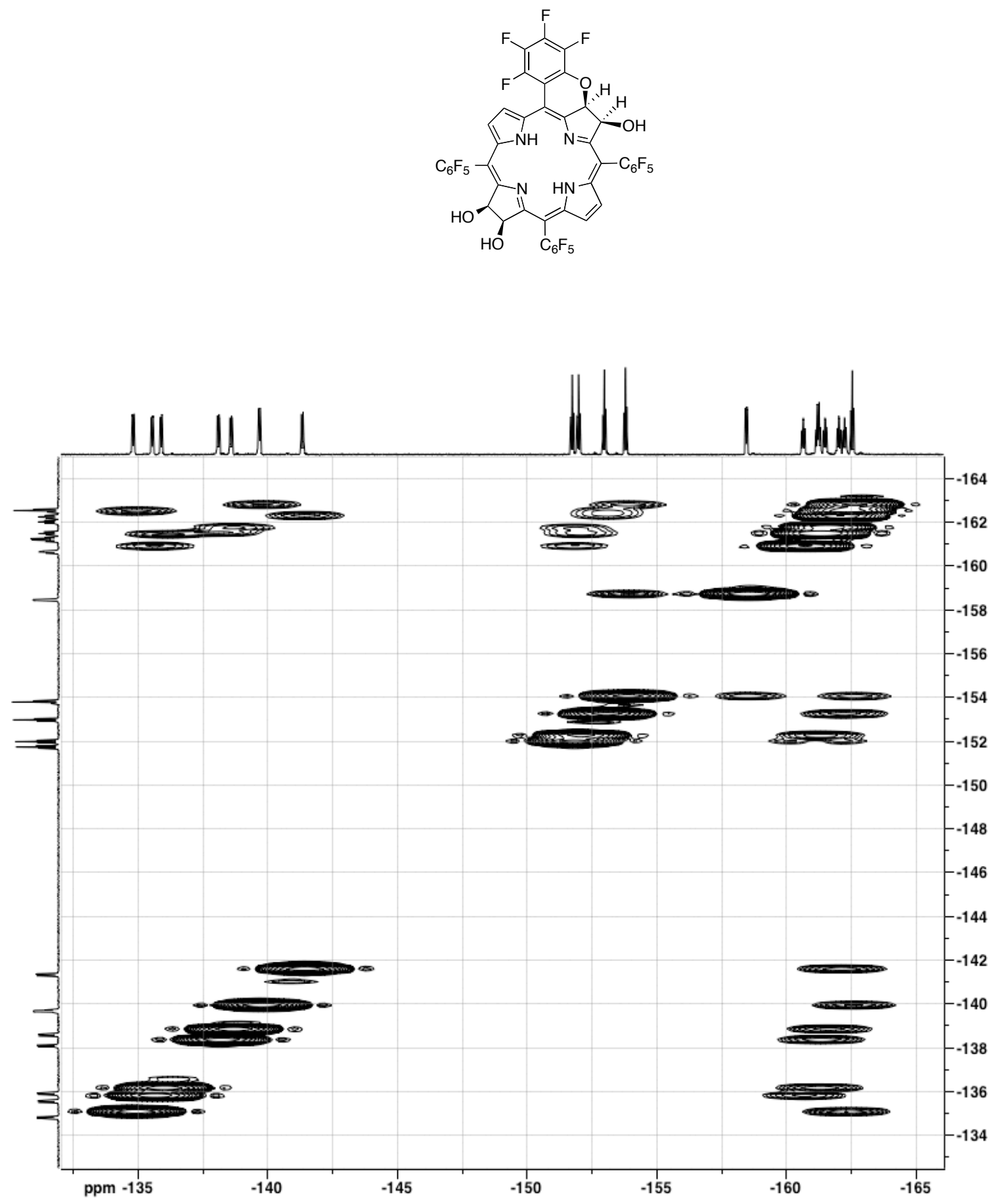

Figure S25. ${ }^{19} \mathrm{~F},{ }^{19} \mathrm{~F}$ QF-COSY NMR spectrum $\left(376 \mathrm{MHz}, \mathrm{CDCl}_{3}\right)$ of $\mathbf{1 0 - Z}$. 

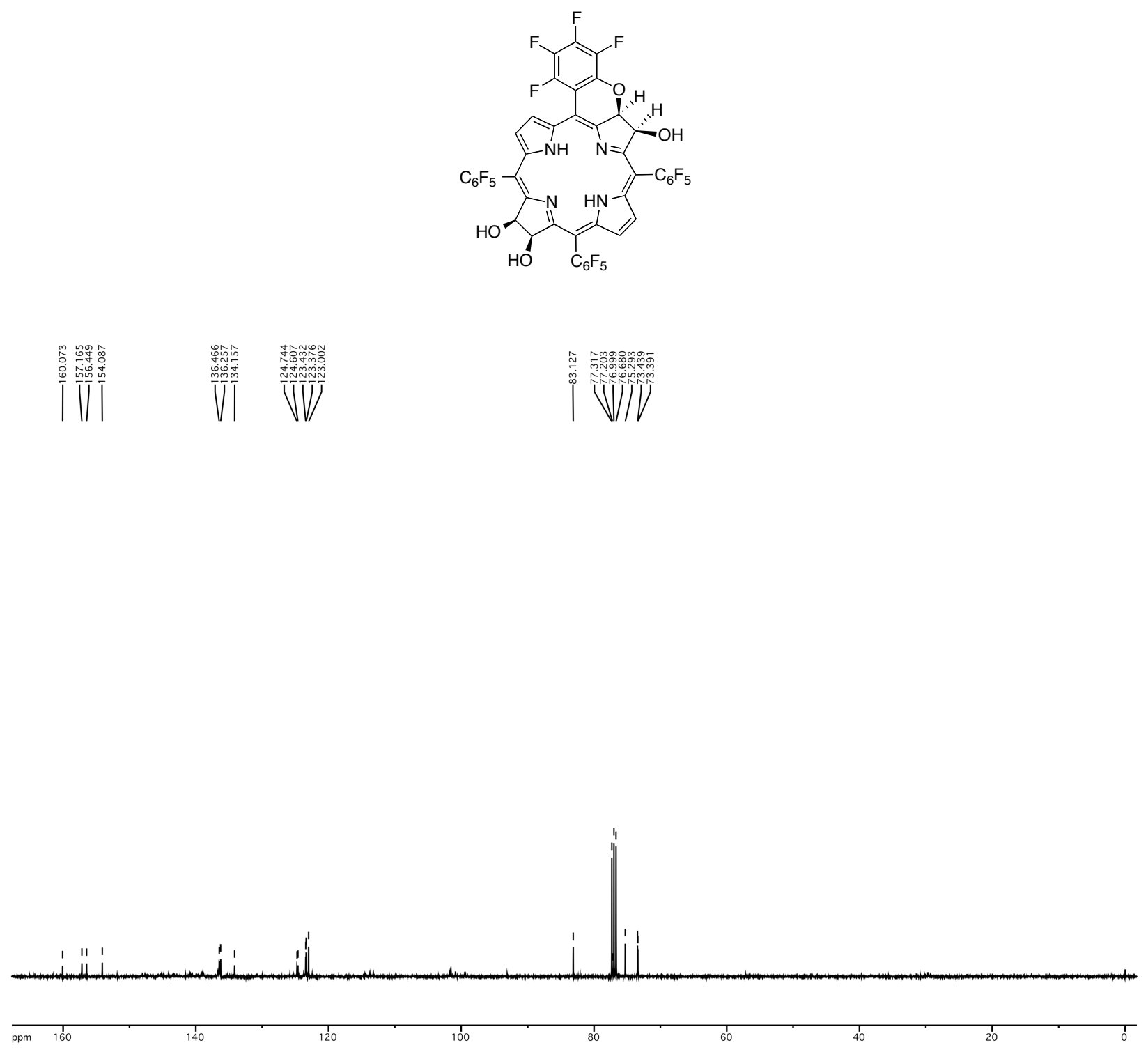

Figure S26. ${ }^{13} \mathrm{C}$ NMR spectrum $\left(100 \mathrm{MHz}, \mathrm{CDCl}_{3}\right)$ of $\mathbf{1 0 -} \boldsymbol{Z}$. 

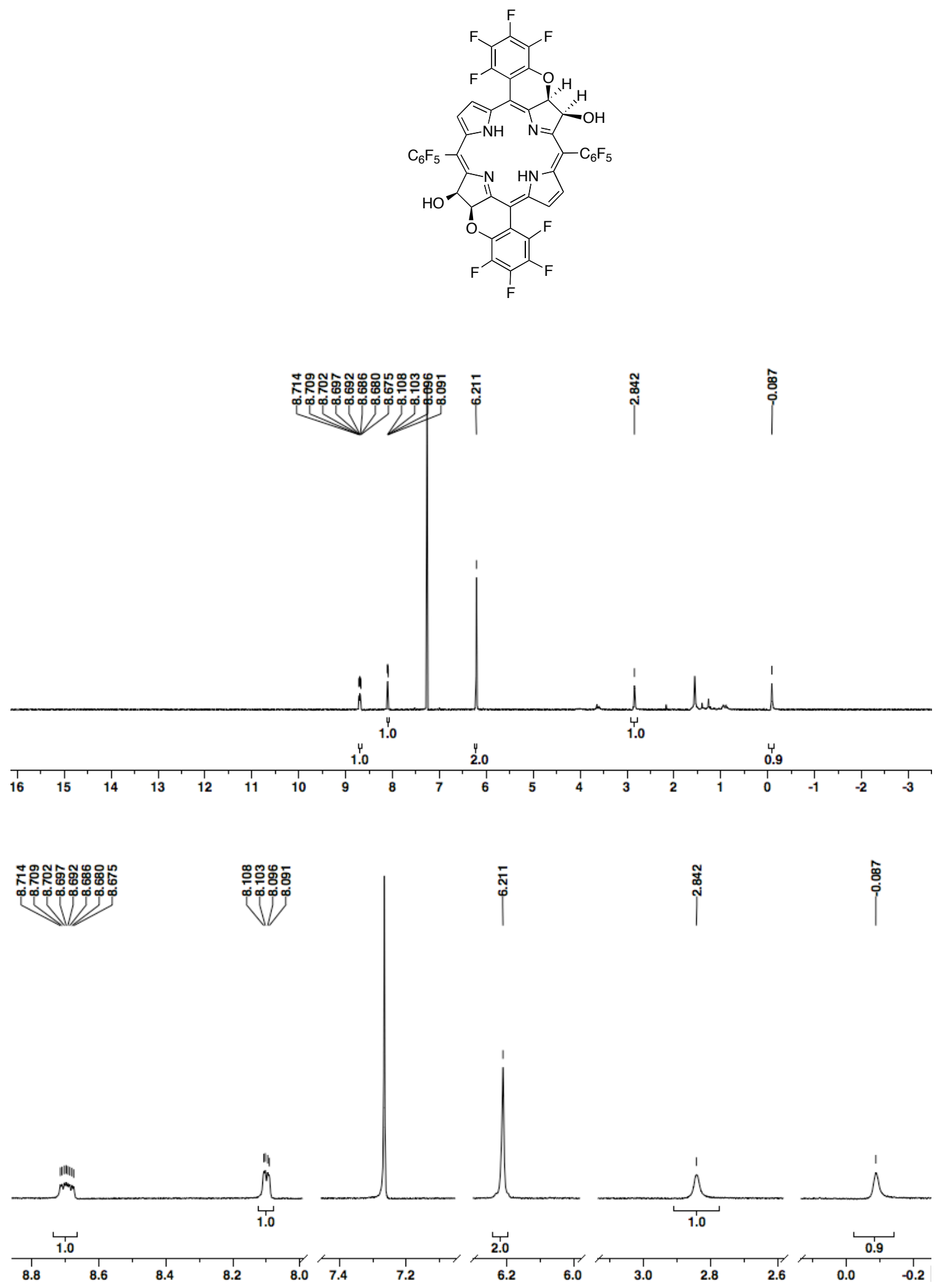

Figure S27. ${ }^{1} \mathrm{H}$ NMR spectrum $\left(400 \mathrm{MHz}, \mathrm{CDCl}_{3}\right)$ of $\mathbf{1 1}^{\mathrm{H}}$-Z-anti. 

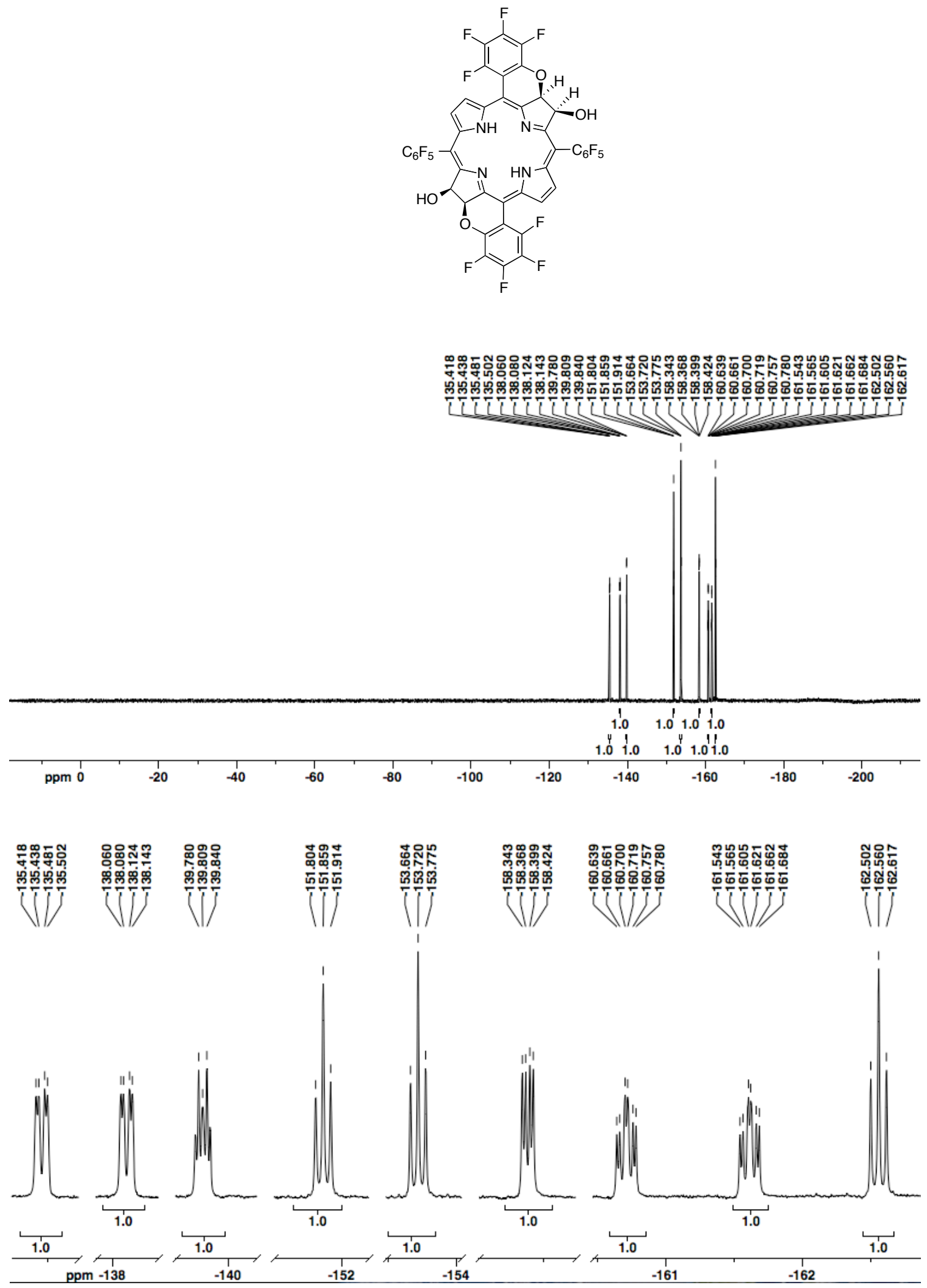

Figure S28. ${ }^{19} \mathrm{~F}$ NMR spectrum $\left(376 \mathrm{MHz}, \mathrm{CDCl}_{3}\right)$ of $\mathbf{1 1}^{\mathrm{H}}$-Z-anti. 

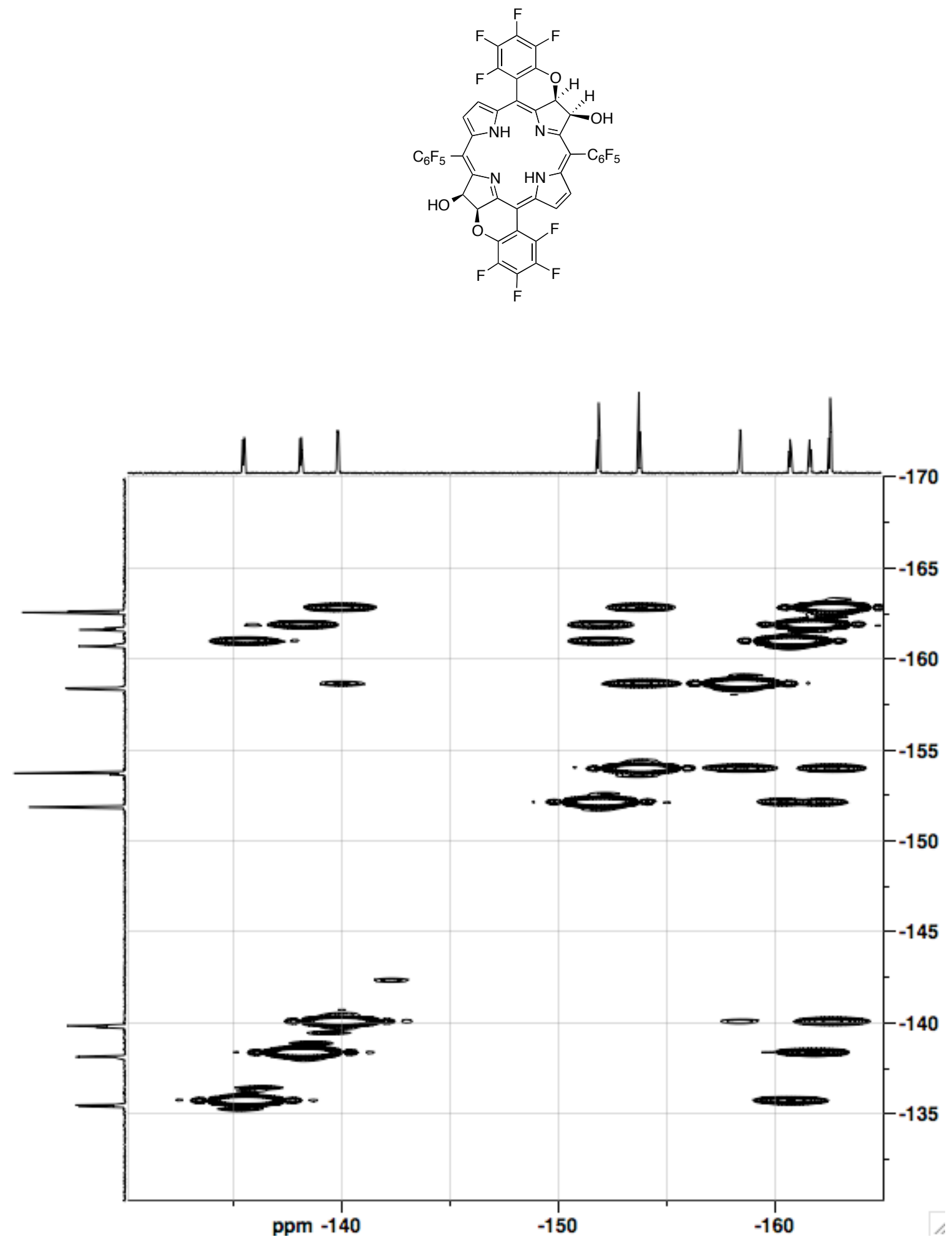

Figure S29. ${ }^{19} \mathrm{~F},{ }^{19} \mathrm{~F}$ QF-COSY NMR spectrum $\left(376 \mathrm{MHz}, \mathrm{CDCl}_{3}\right)$ of $\mathbf{1 1}^{\mathbf{H}}$-Z-anti. 

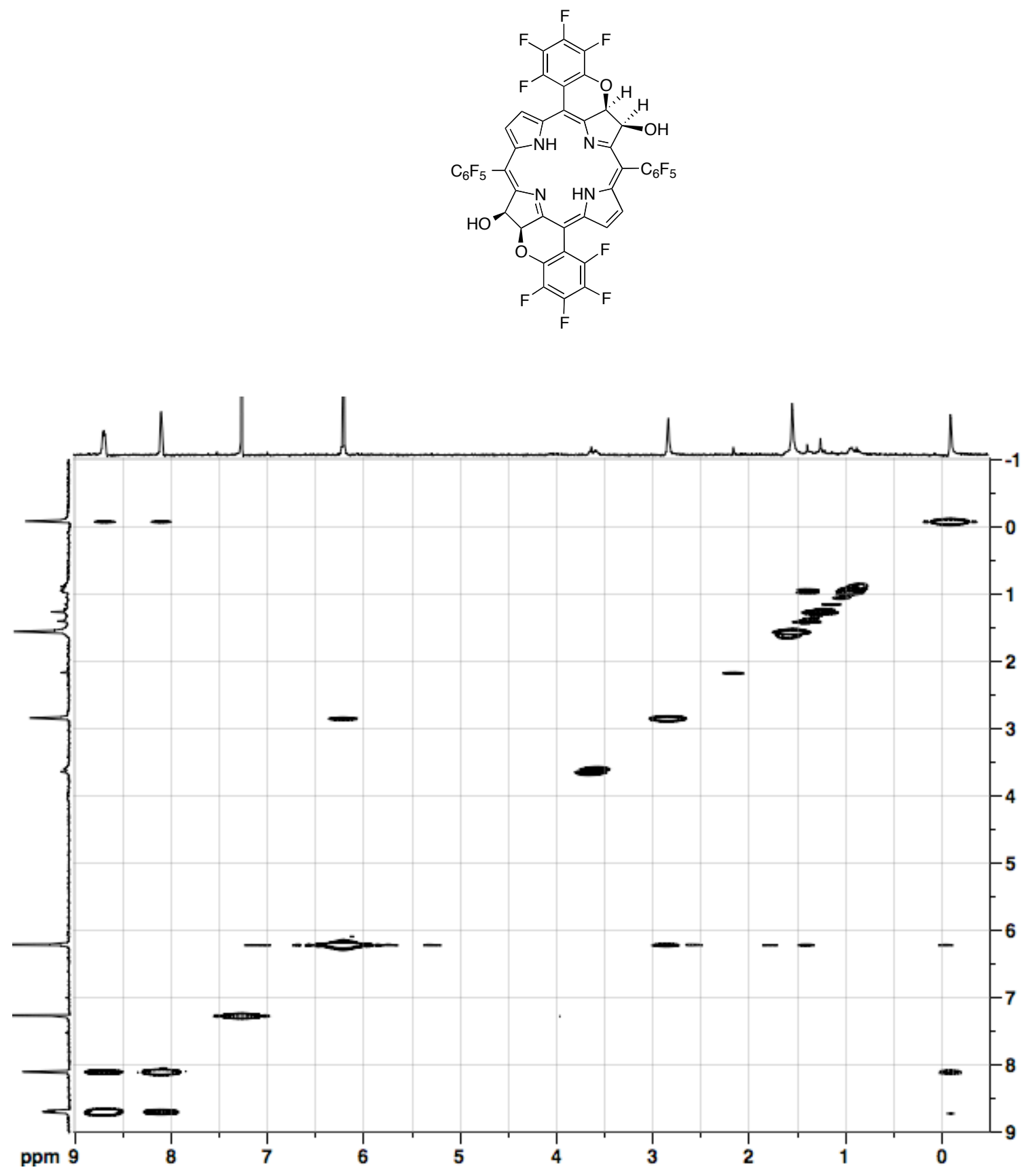

Figure S30. ${ }^{1} \mathrm{H},{ }^{1} \mathrm{H}$ COSY NMR spectrum $\left(400 \mathrm{MHz}, \mathrm{CDCl}_{3}\right)$ of $\mathbf{1 1}^{\mathbf{H}}$-Z-anti. 

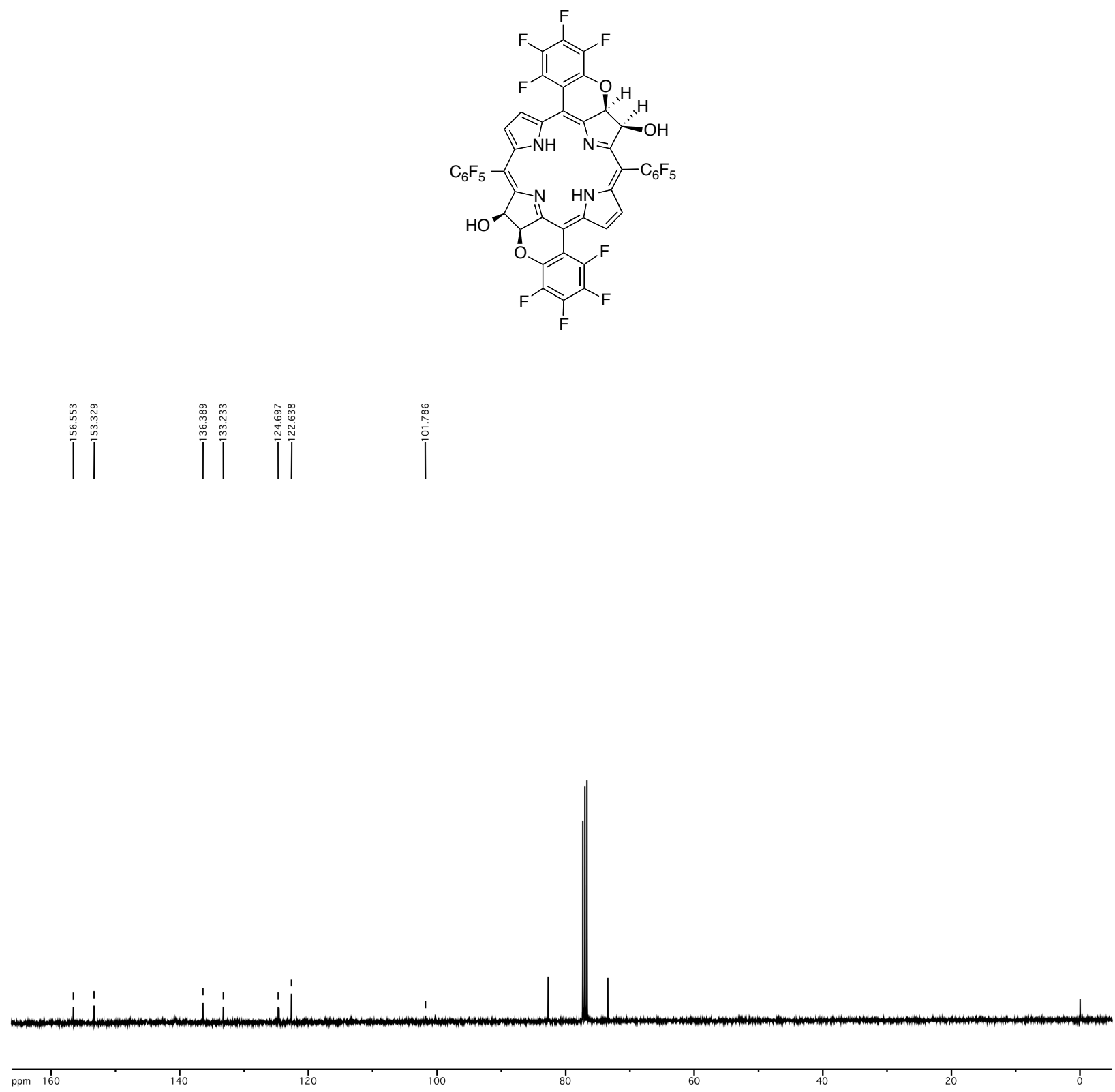

Figure S31. ${ }^{13} \mathrm{C}$ NMR spectrum $\left(100 \mathrm{MHz}, \mathrm{CDCl}_{3}\right)$ of $\mathbf{1 1}^{\mathrm{H}}-\boldsymbol{Z}$-anti. 

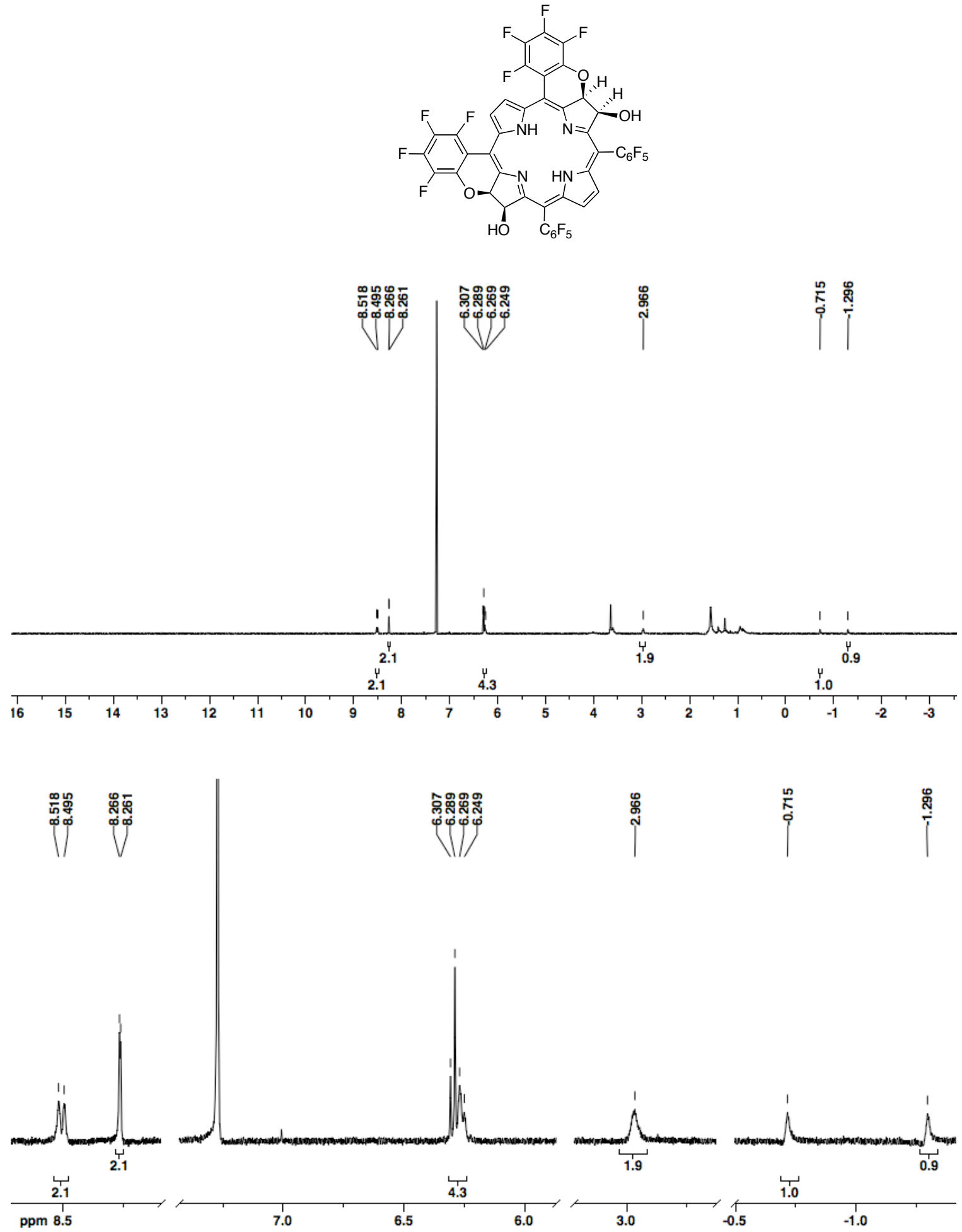

Figure S32. ${ }^{1} \mathrm{H}$ NMR spectrum $\left(400 \mathrm{MHz}, \mathrm{CDCl}_{3}\right)$ of $\mathbf{1 1}^{\mathbf{H}}-\boldsymbol{Z}$-syn . 

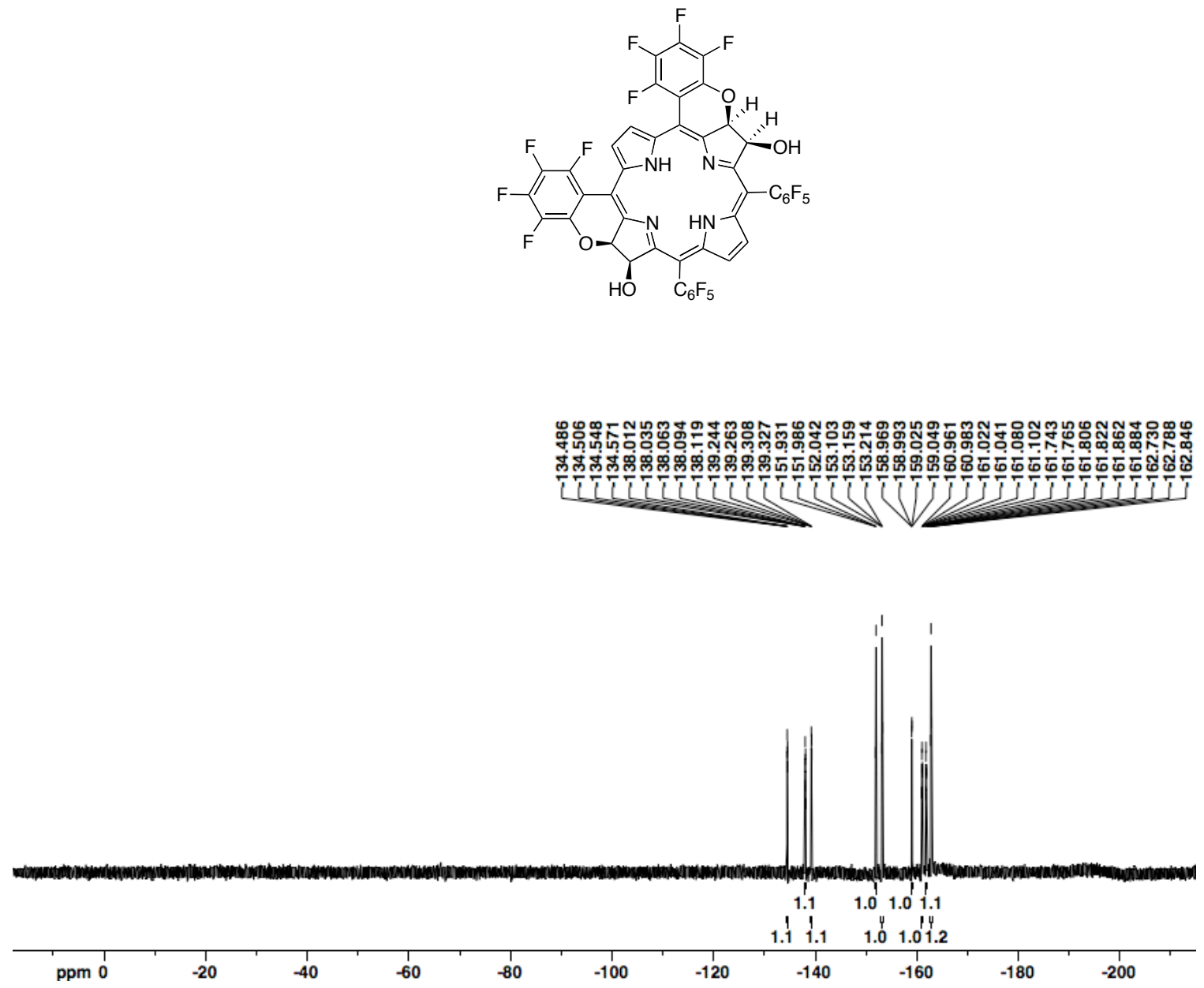

\begin{tabular}{|c|c|c|c|c|c|c|}
\hline Vij & 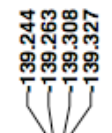 & 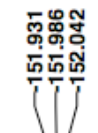 & 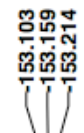 & 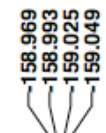 & 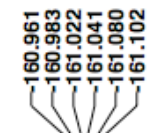 & 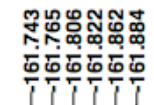 \\
\hline
\end{tabular}

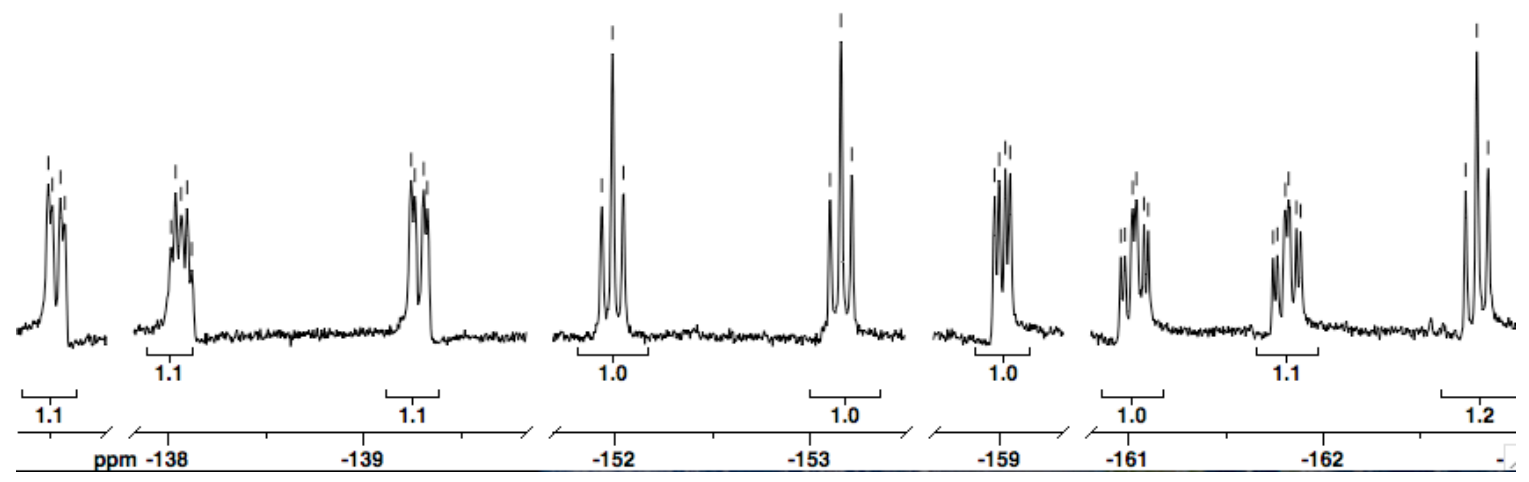

Figure S33. ${ }^{19} \mathrm{~F}$ NMR spectrum $\left(376 \mathrm{MHz}, \mathrm{CDCl}_{3}\right)$ of $\mathbf{1 1}^{\mathrm{H}}-\mathbf{Z}$-syn . 

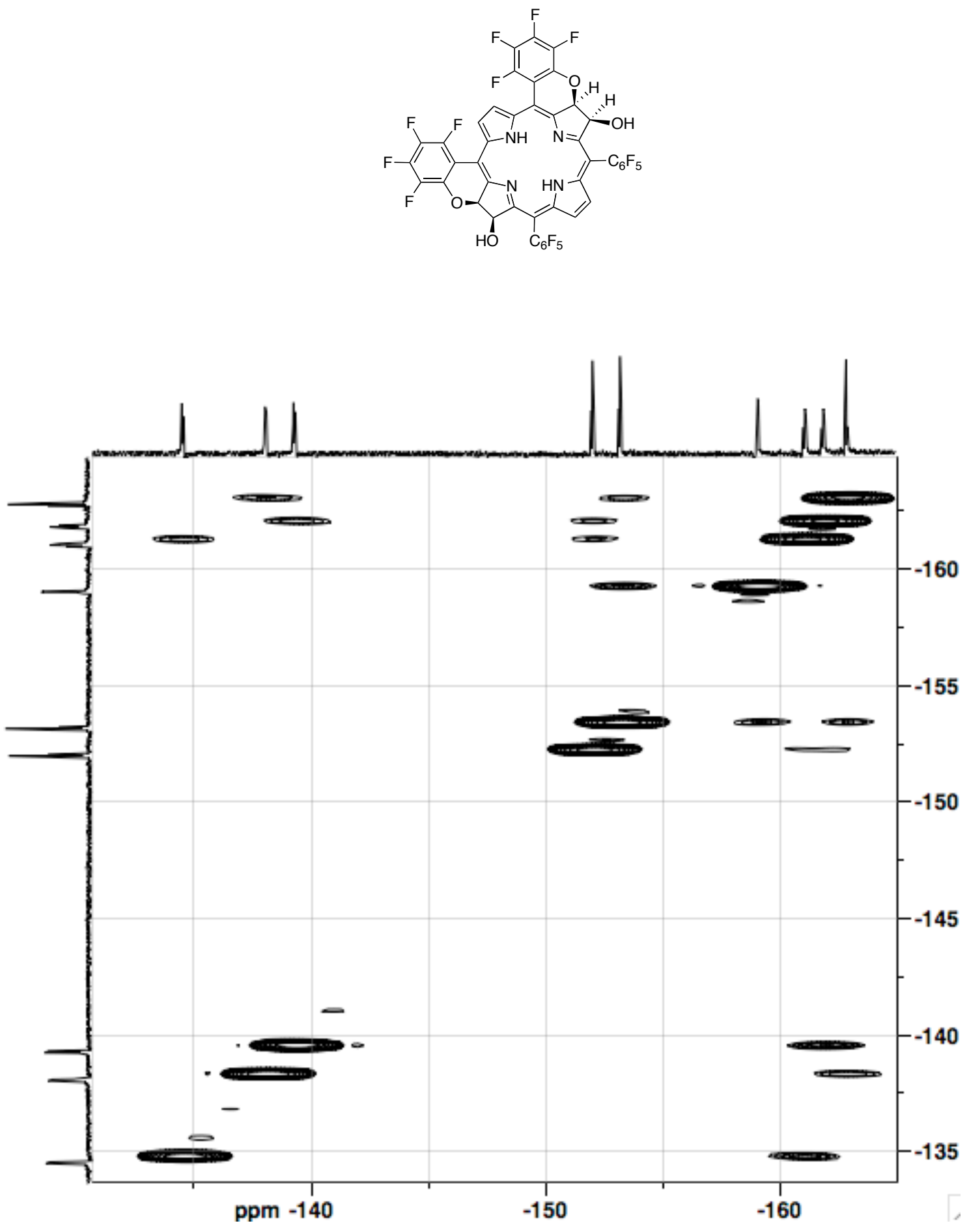

Figure S34. ${ }^{19} \mathrm{~F},{ }^{19} \mathrm{~F}$ QF-COSY NMR spectrum $\left(376 \mathrm{MHz}, \mathrm{CDCl}_{3}\right)$ of $\mathbf{1 1}^{\mathbf{H}}-\boldsymbol{Z}$-syn . 

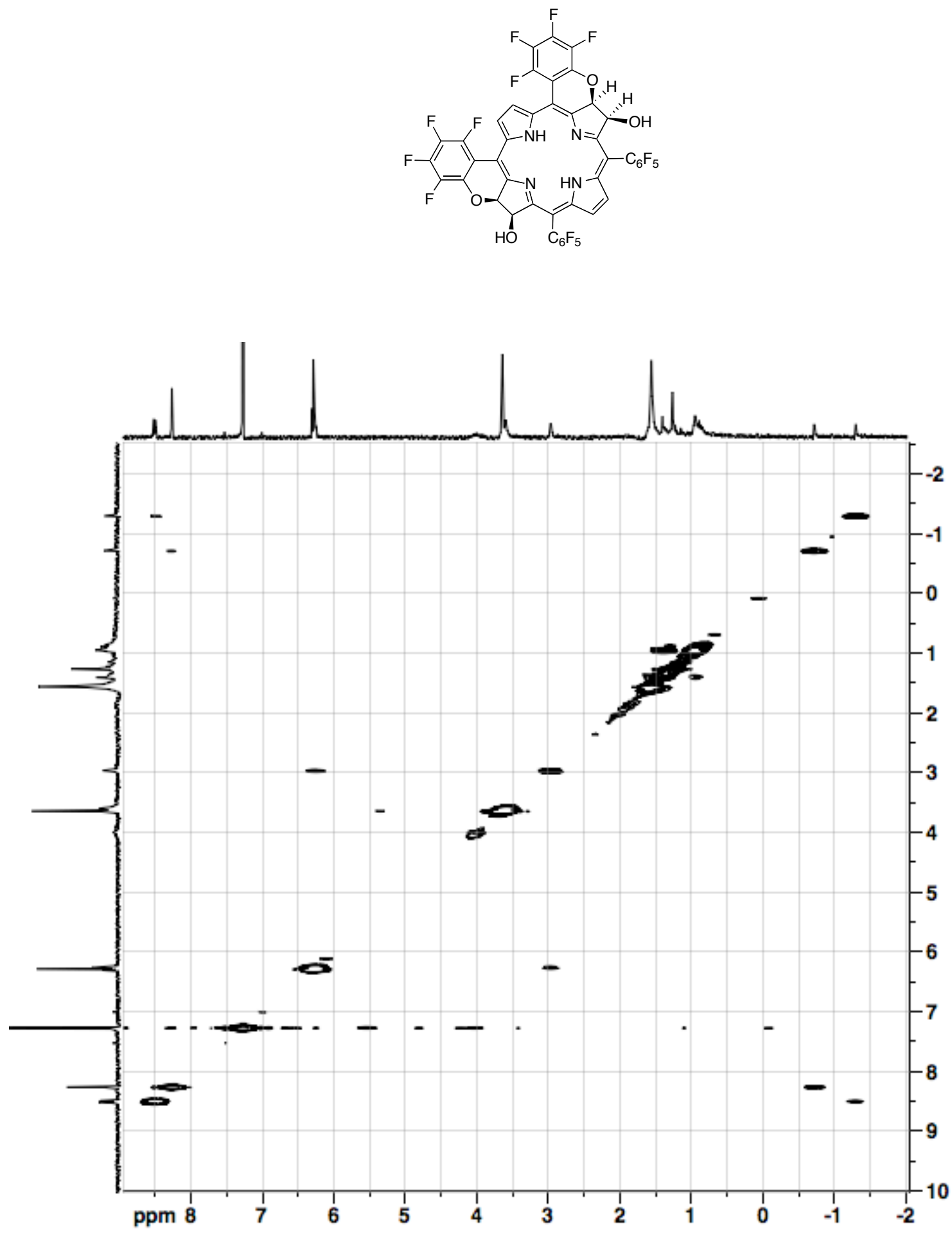

Figure S35. ${ }^{1} \mathrm{H},{ }^{1} \mathrm{H}$ COSY NMR spectrum $\left(400 \mathrm{MHz}, \mathrm{CDCl}_{3}\right)$ of $\mathbf{1 1}^{\mathbf{H}} \mathbf{- Z}$-syn . 

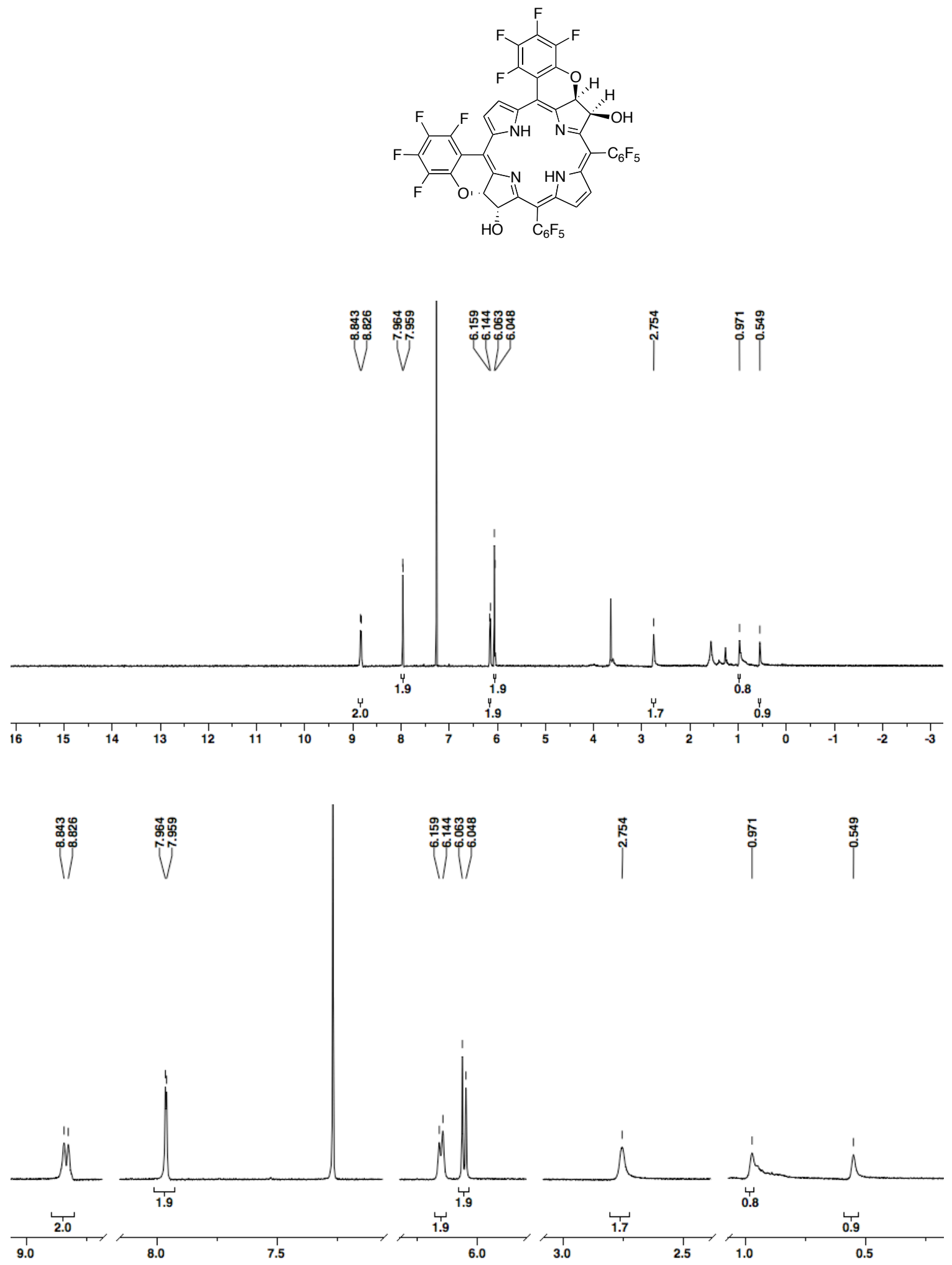

Figure S36. ${ }^{1} \mathrm{H}$ NMR spectrum $\left(400 \mathrm{MHz}, \mathrm{CDCl}_{3}\right)$ of $\mathbf{1 1}^{\mathrm{H}}$ - $\boldsymbol{E}$-syn . 

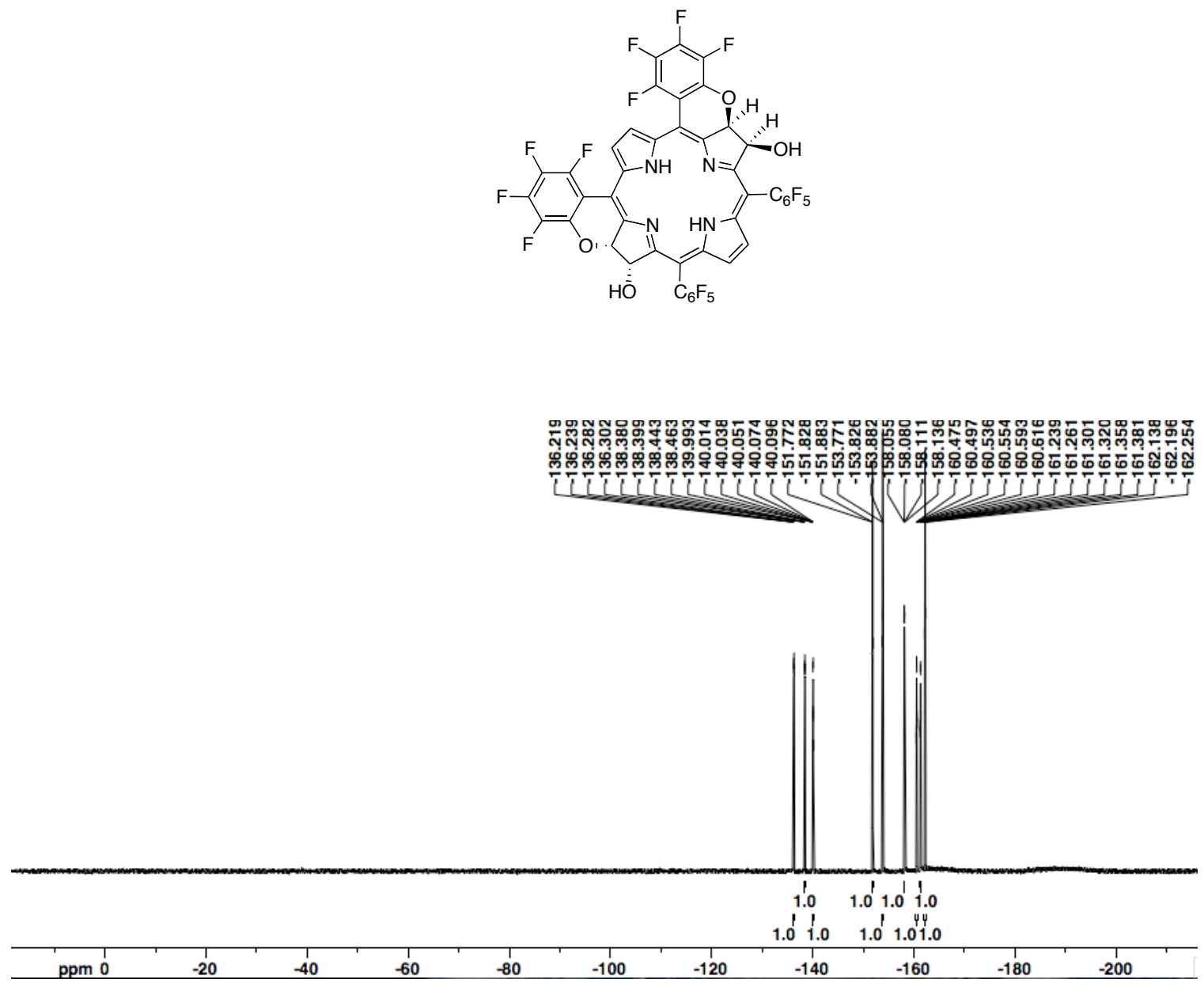

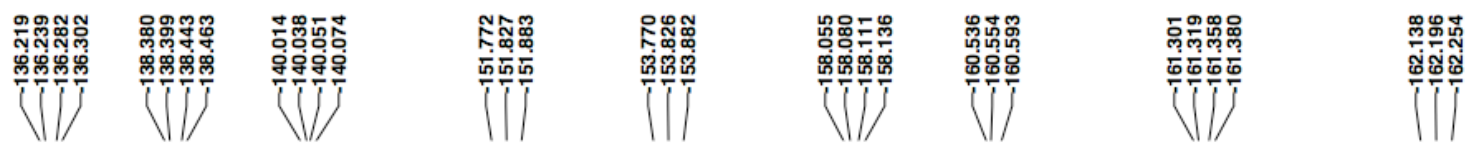

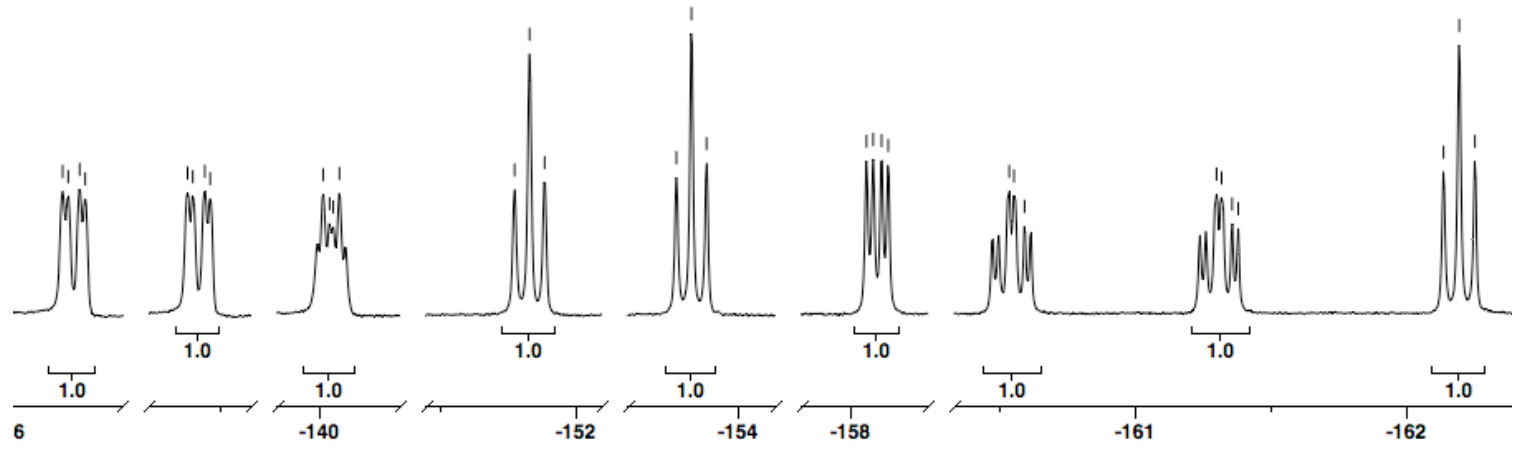

Figure S37. ${ }^{19} \mathrm{~F}$ NMR spectrum $\left(376 \mathrm{MHz}, \mathrm{CDCl}_{3}\right)$ of $\mathbf{1 1}^{\mathbf{H}} \mathbf{- E}$-syn. 

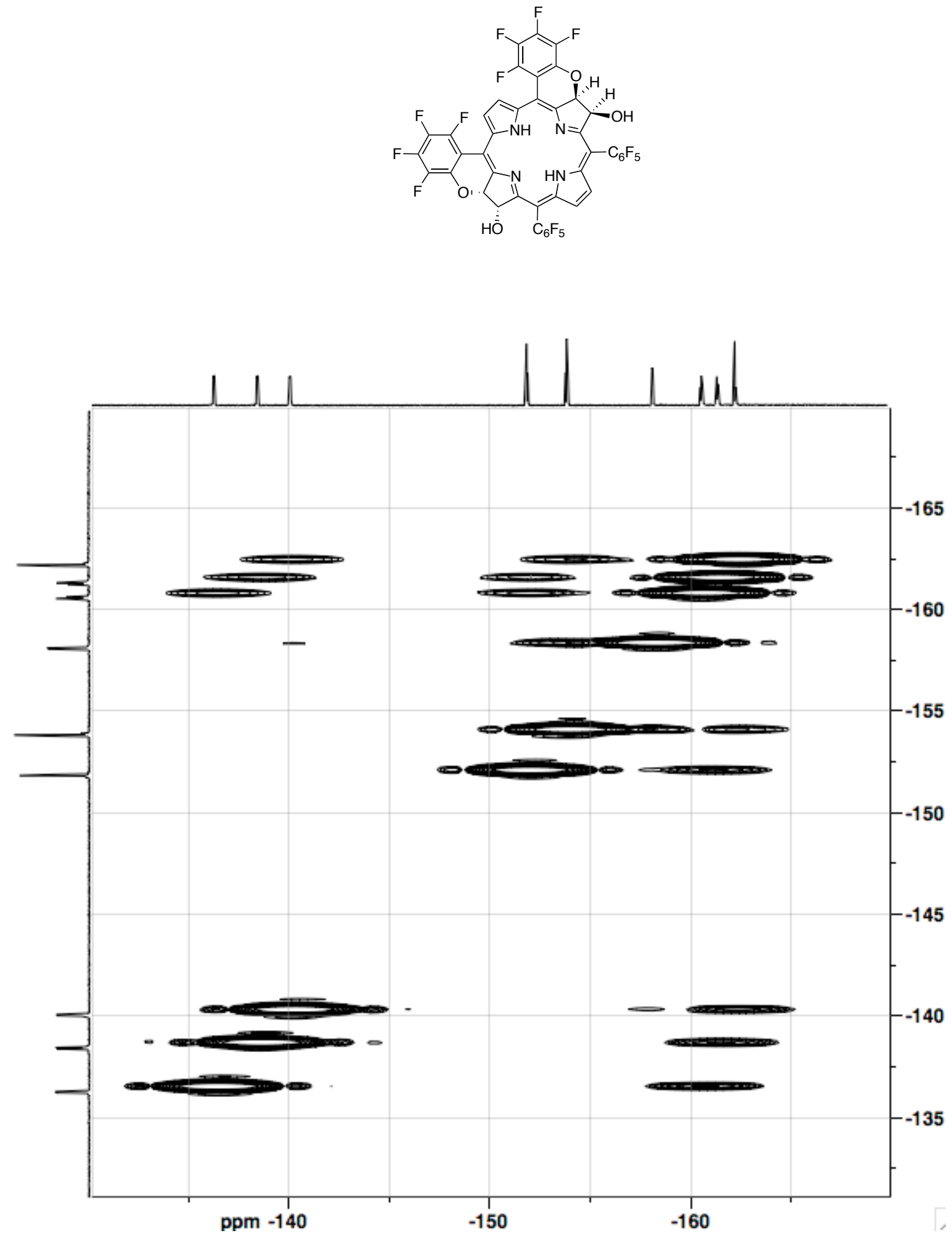

Figure S38. ${ }^{19} \mathrm{~F},{ }^{19} \mathrm{~F}$ QF-COSY NMR spectrum $\left(376 \mathrm{MHz}, \mathrm{CDCl}_{3}\right)$ of $\mathbf{1 1}^{\mathbf{H}}-\boldsymbol{E}$-syn. 

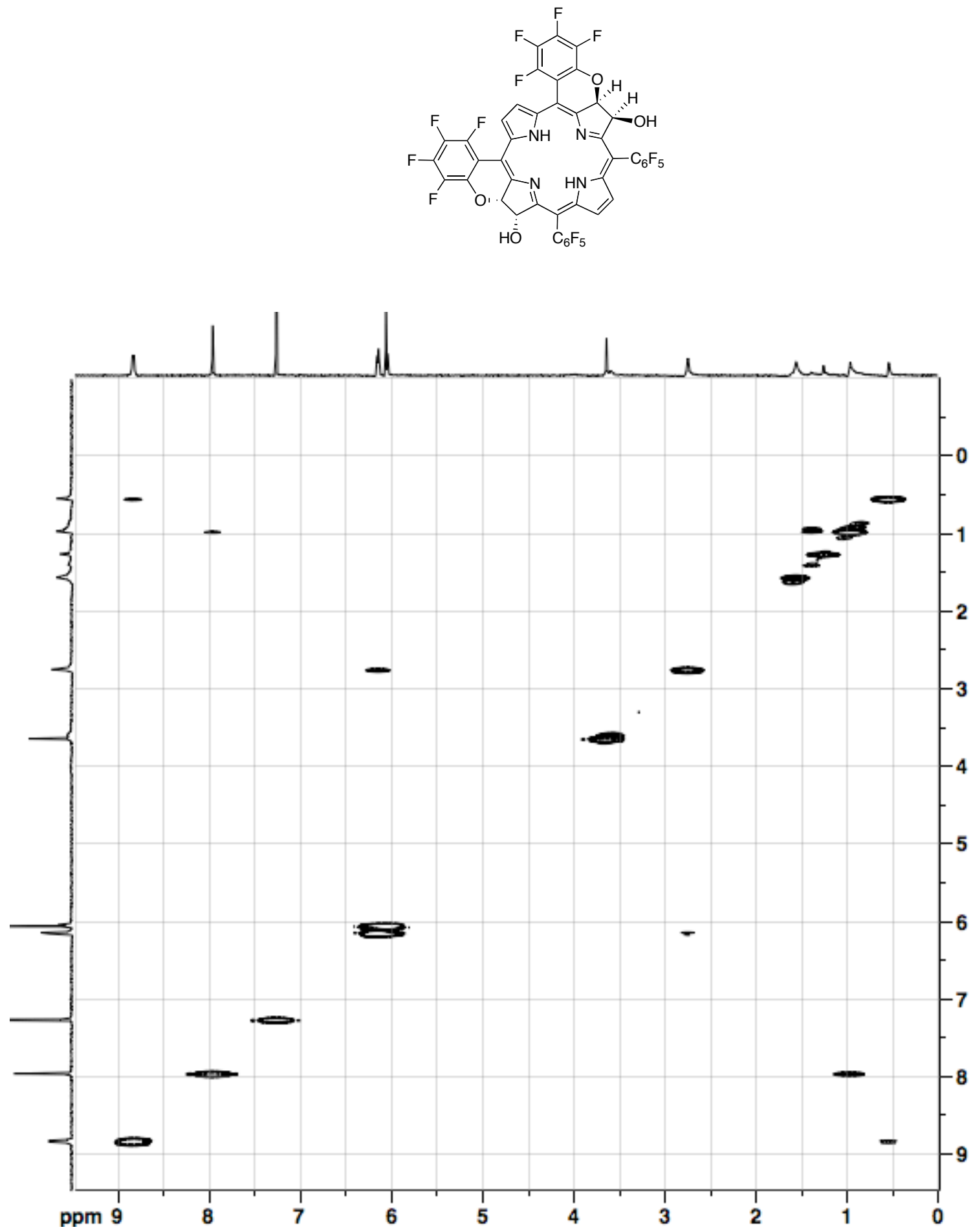

Figure S39. ${ }^{1} \mathrm{H},{ }^{1} \mathrm{H}$ COSY NMR spectrum $\left(400 \mathrm{MHz}, \mathrm{CDCl}_{3}\right)$ of $\mathbf{1 1}^{\mathbf{H}} \mathbf{- E}$-syn . 

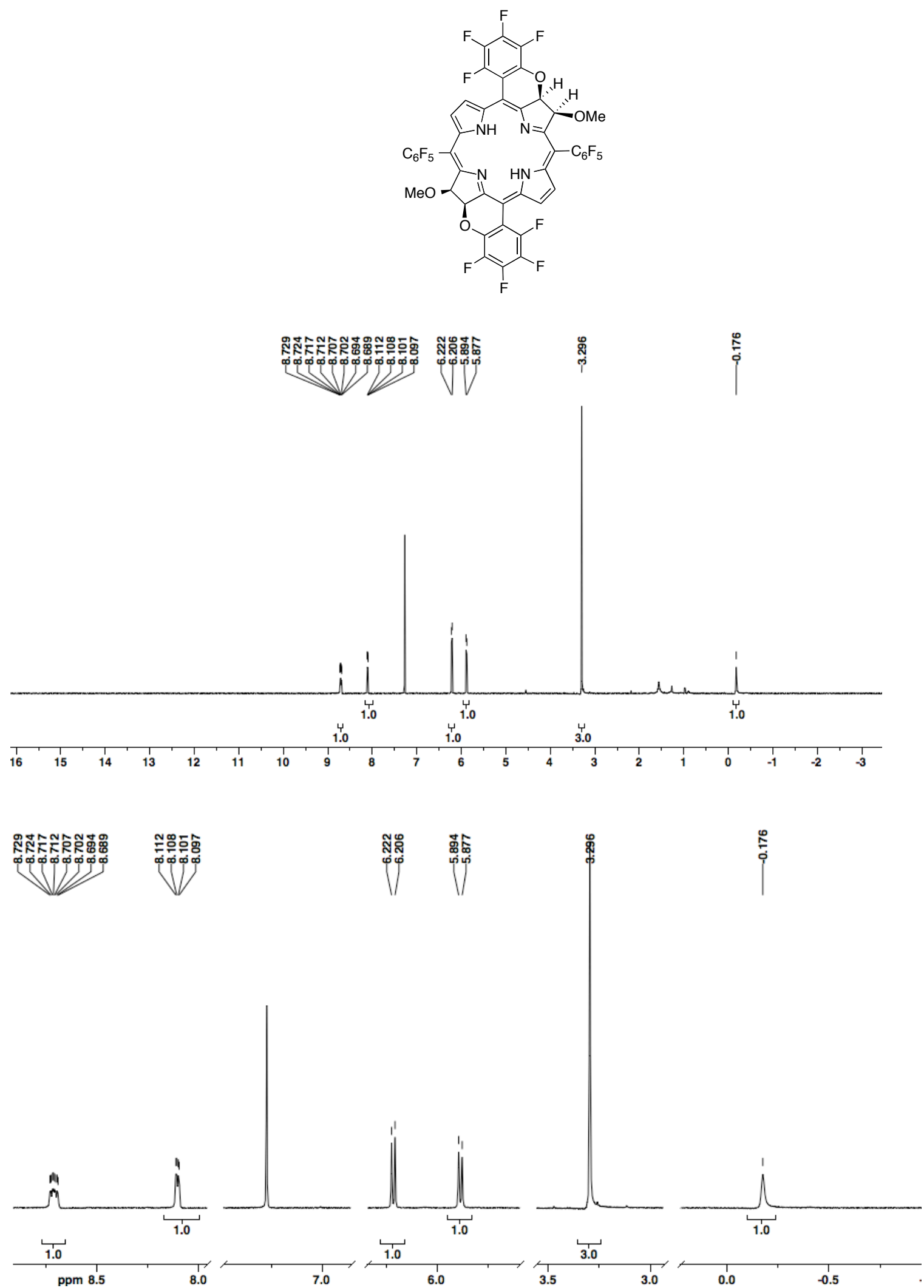

Figure S40. ${ }^{1} \mathrm{H}$ NMR spectrum $\left(400 \mathrm{MHz}, \mathrm{CDCl}_{3}\right)$ of $\mathbf{1 1 ^ { \mathrm { Me } }}-\boldsymbol{Z}$-anti. 

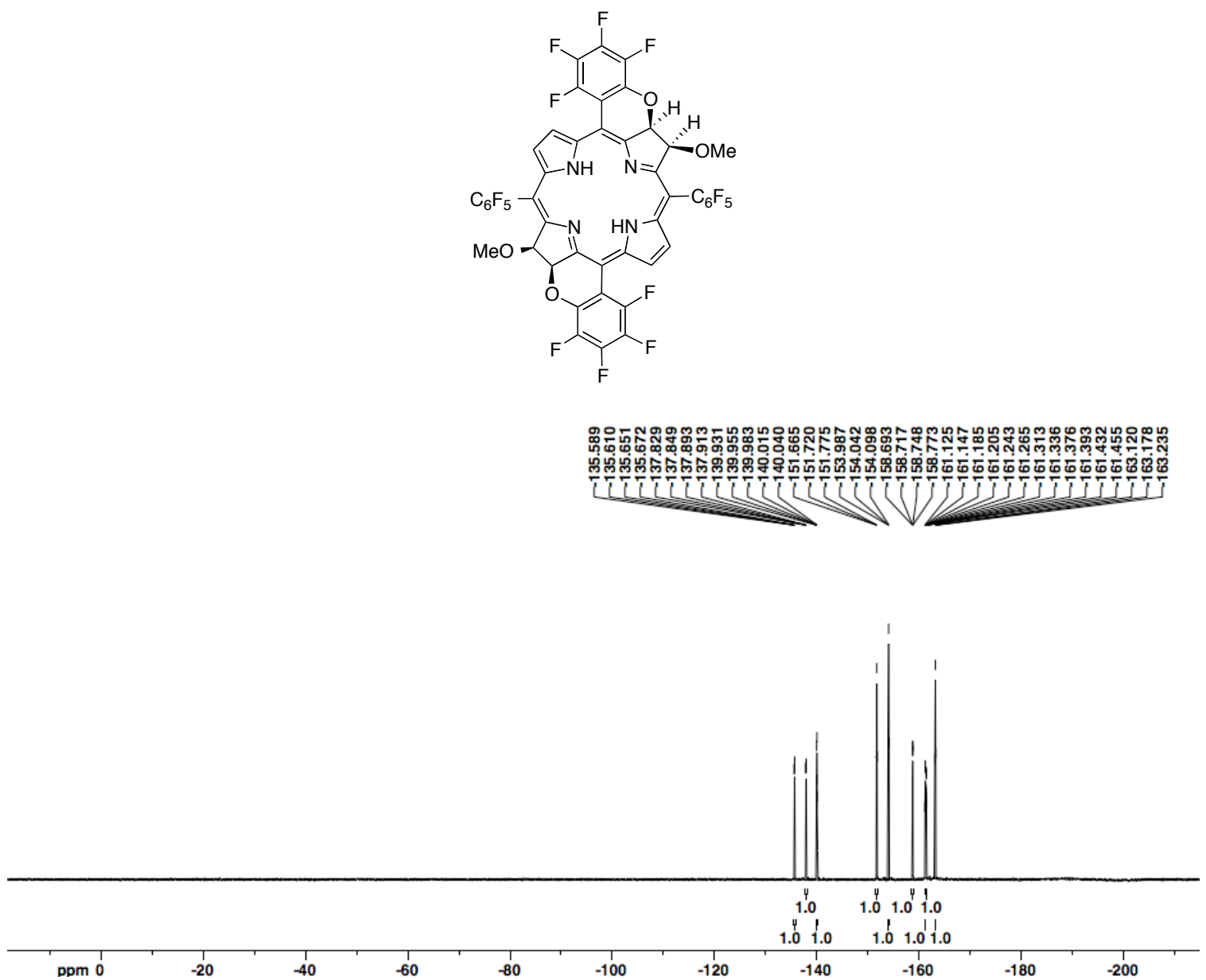

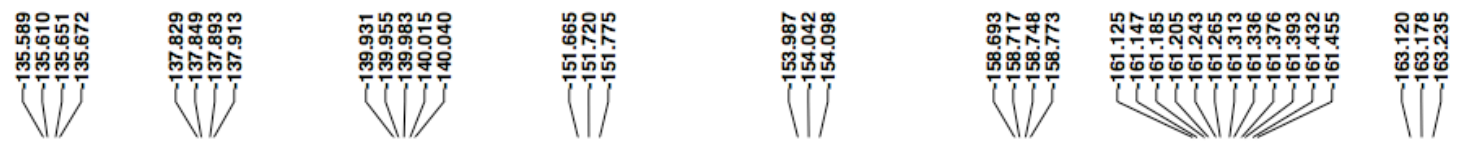
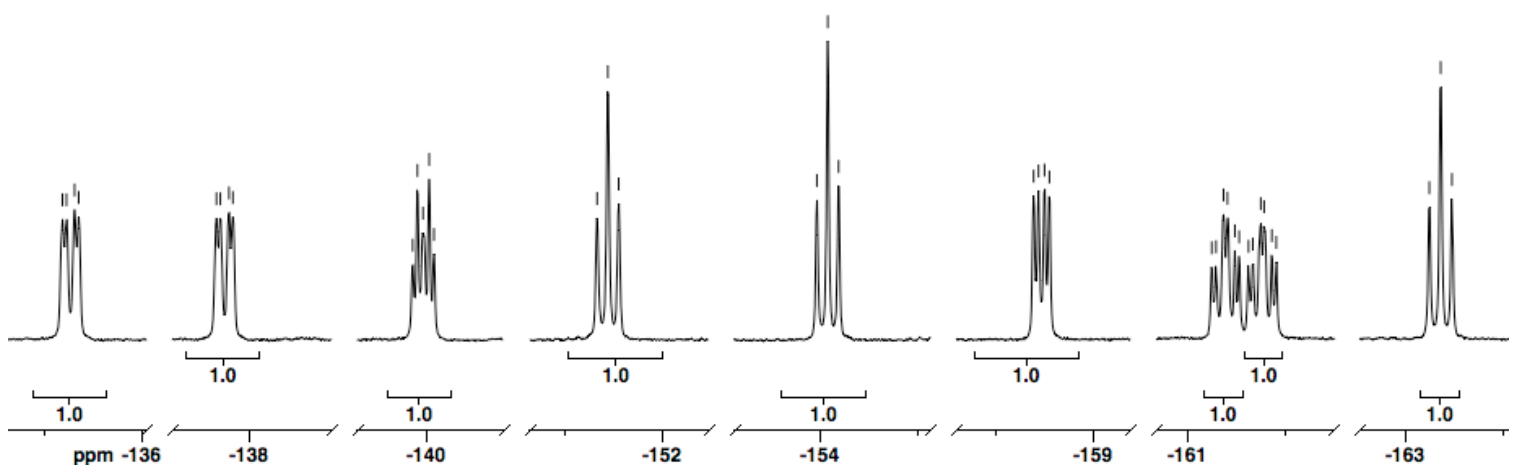

Figure S41. ${ }^{19} \mathrm{~F}$ NMR spectrum $\left(376 \mathrm{MHz}, \mathrm{CDCl}_{3}\right)$ of $\mathbf{1 1}{ }^{\mathrm{Me}}$-Z-anti. 

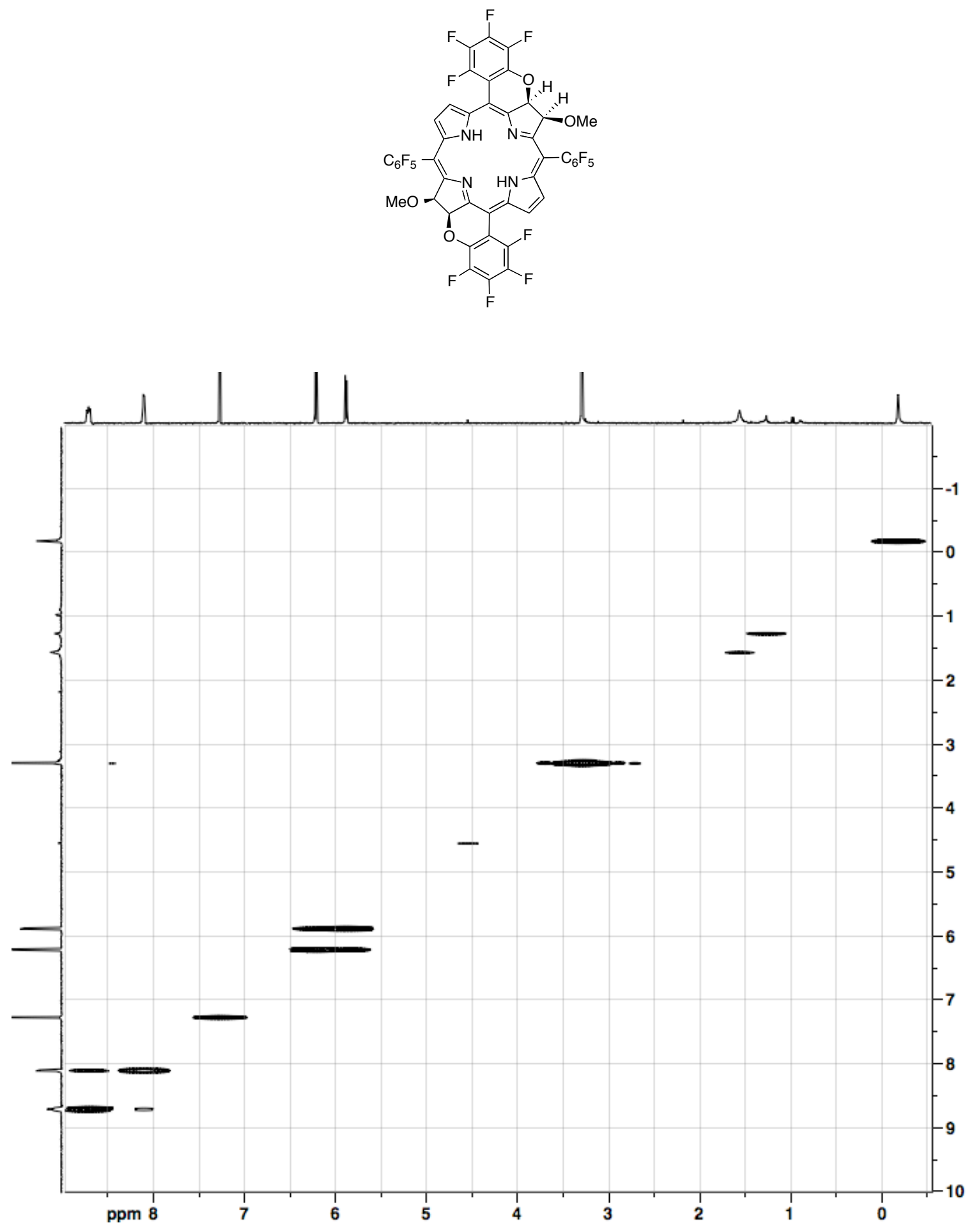

Figure S42. ${ }^{1} \mathrm{H},{ }^{1} \mathrm{H}-\mathrm{COSY}$ NMR spectrum $\left(400 \mathrm{MHz}, \mathrm{CDCl}_{3}\right)$ of $\mathbf{1 1}^{\mathrm{Me}}$-Z-anti. 

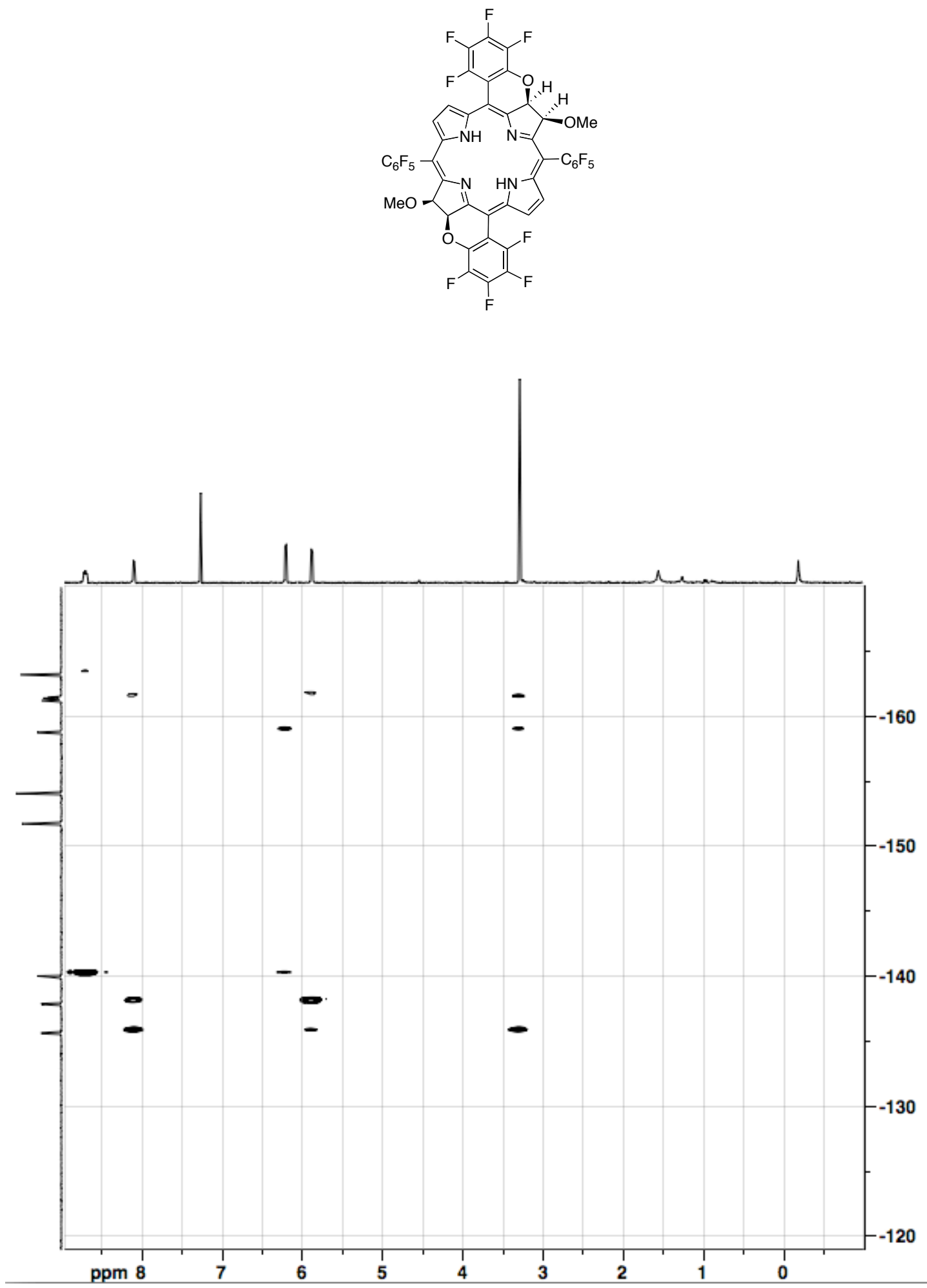

Figure S43. ${ }^{1} \mathrm{H},{ }^{19} \mathrm{~F}$-HOESY NMR spectrum $\left(400,376 \mathrm{MHz}, \mathrm{CDCl}_{3}\right)$ of $\mathbf{1 1}^{\mathrm{Me}}-$ Z-anti. 

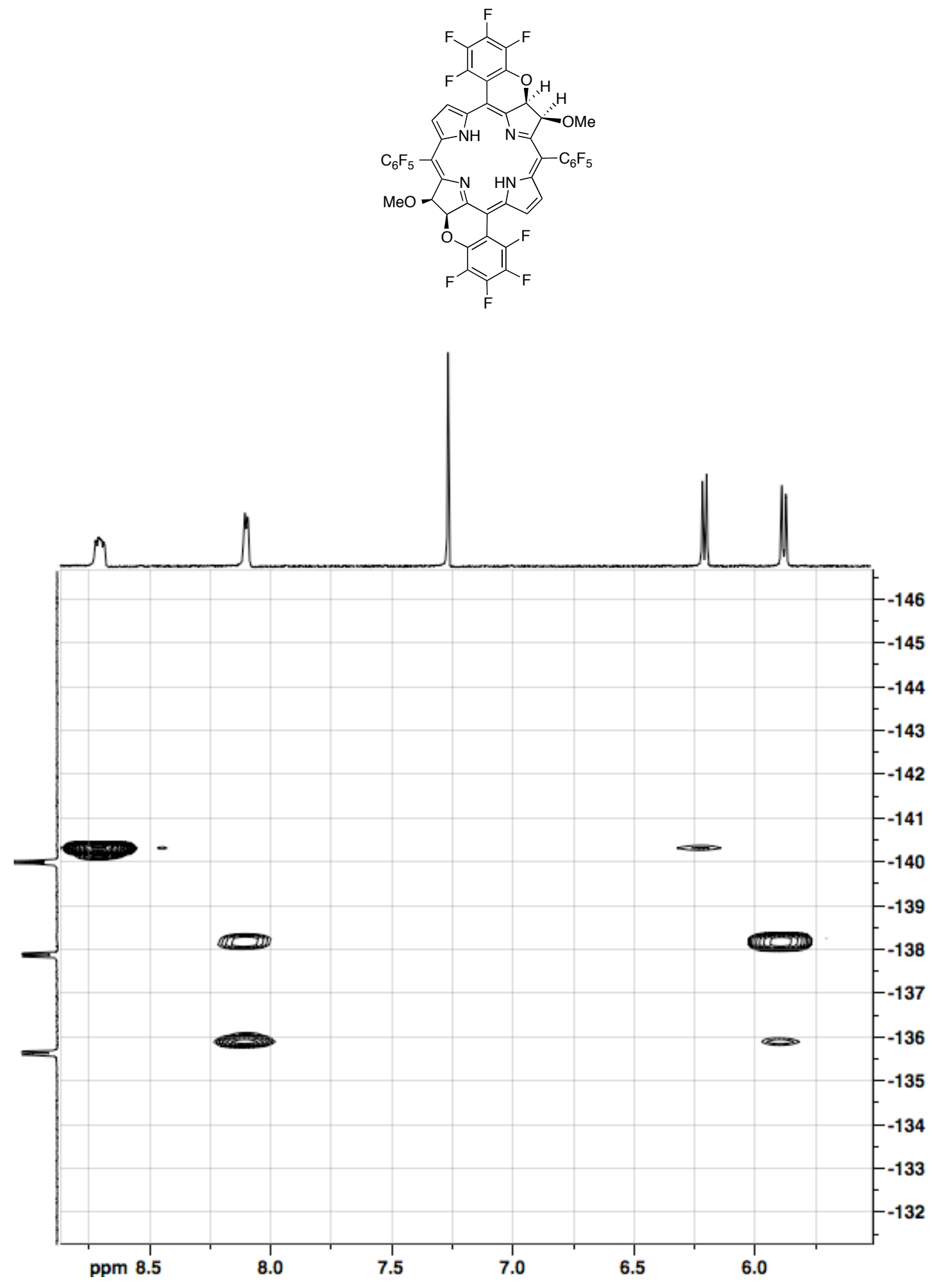

Figure S44. Partial ${ }^{1} \mathrm{H},{ }^{19} \mathrm{~F}-\mathrm{HOESY}$ NMR spectrum $\left(400,376 \mathrm{MHz}, \mathrm{CDCl}_{3}\right)$ of $\mathbf{1 1}^{\mathrm{Me}}$ - $\boldsymbol{Z}$-anti. 

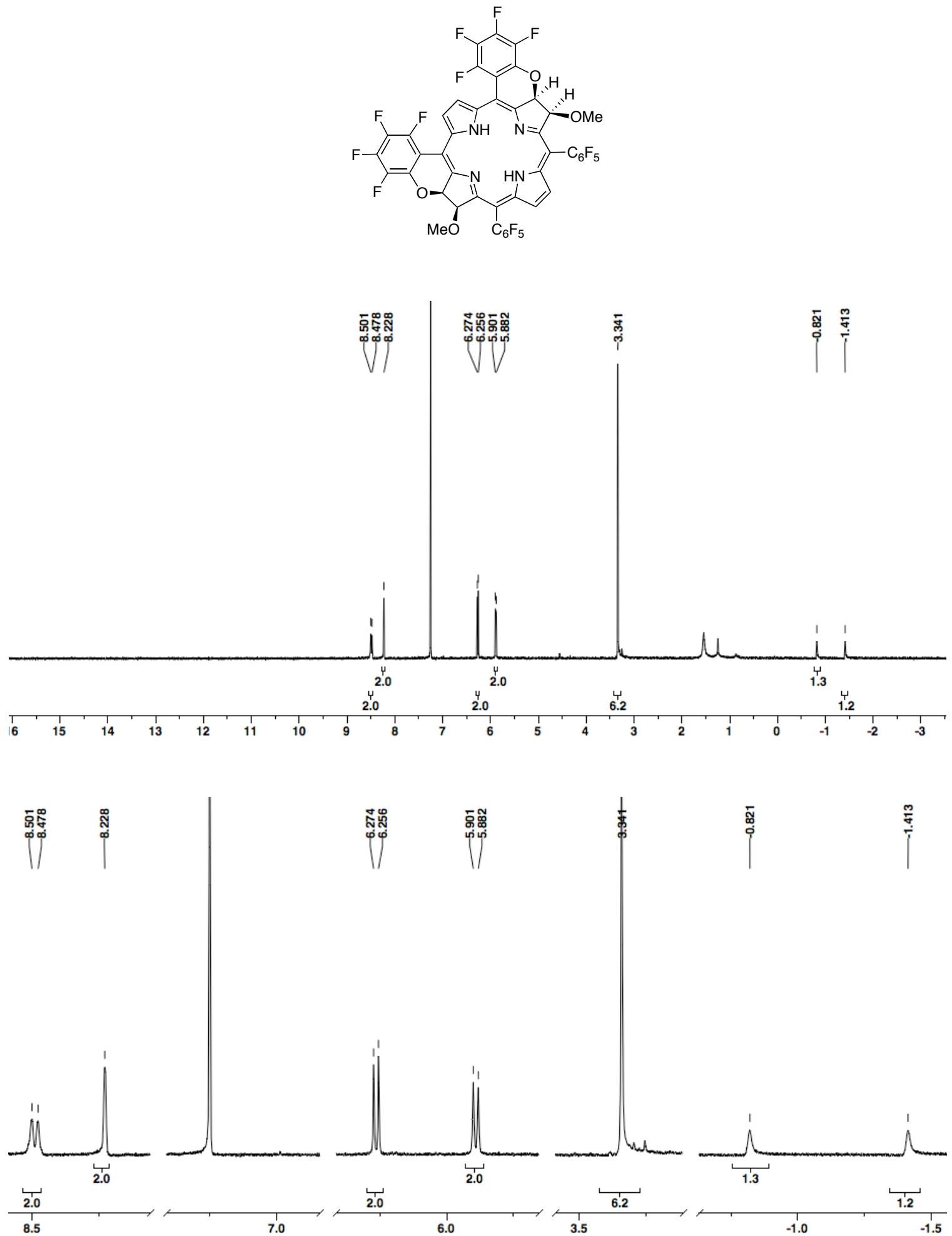

Figure S45. ${ }^{1} \mathrm{H}$ NMR spectrum $\left(400 \mathrm{MHz}, \mathrm{CDCl}_{3}\right)$ of $\mathbf{1 1}{ }^{\mathrm{Me}}-\boldsymbol{Z}$-syn . 

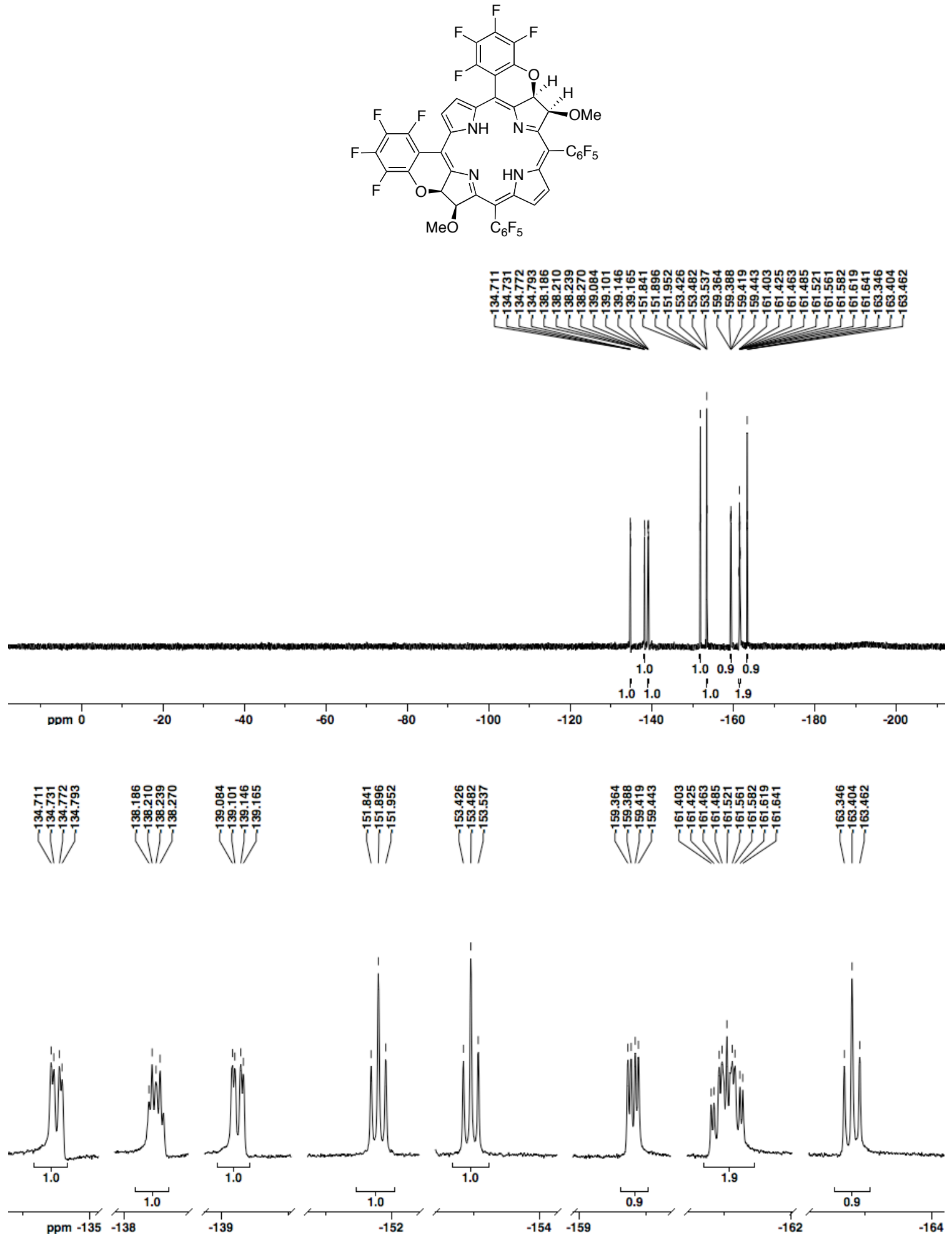

Figure S46. ${ }^{19} \mathrm{~F}$ NMR spectrum $\left(376 \mathrm{MHz}, \mathrm{CDCl}_{3}\right)$ of $\mathbf{1 1}^{\mathrm{Me}}-\boldsymbol{Z}$-syn . 

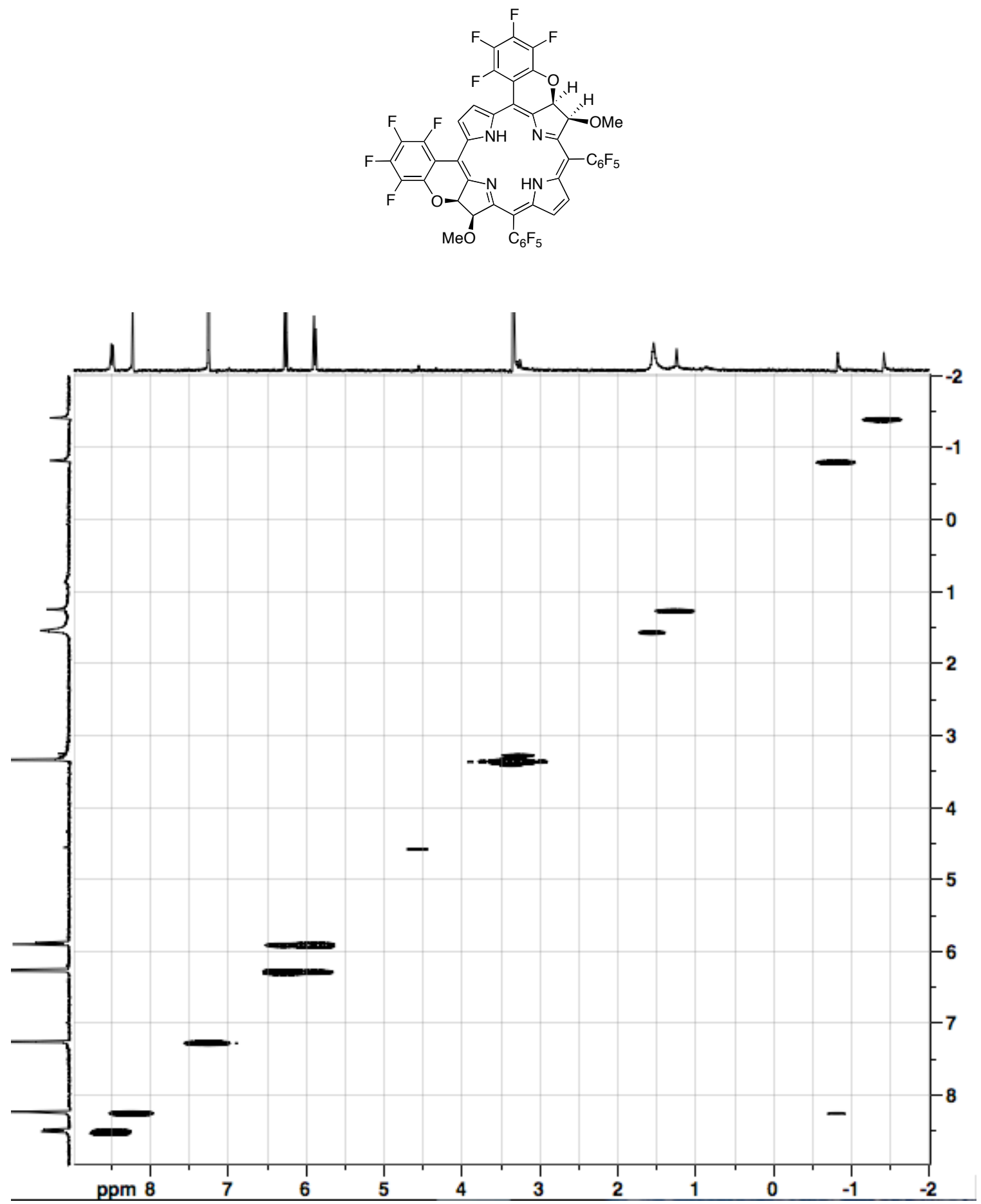

Figure S47. ${ }^{1} \mathrm{H},{ }^{1} \mathrm{H}-\mathrm{COSY} \mathrm{NMR}$ spectrum $\left(400 \mathrm{MHz}, \mathrm{CDCl}_{3}\right)$ of $\mathbf{1 1}^{\mathrm{Me}}-\boldsymbol{Z}$-syn . 

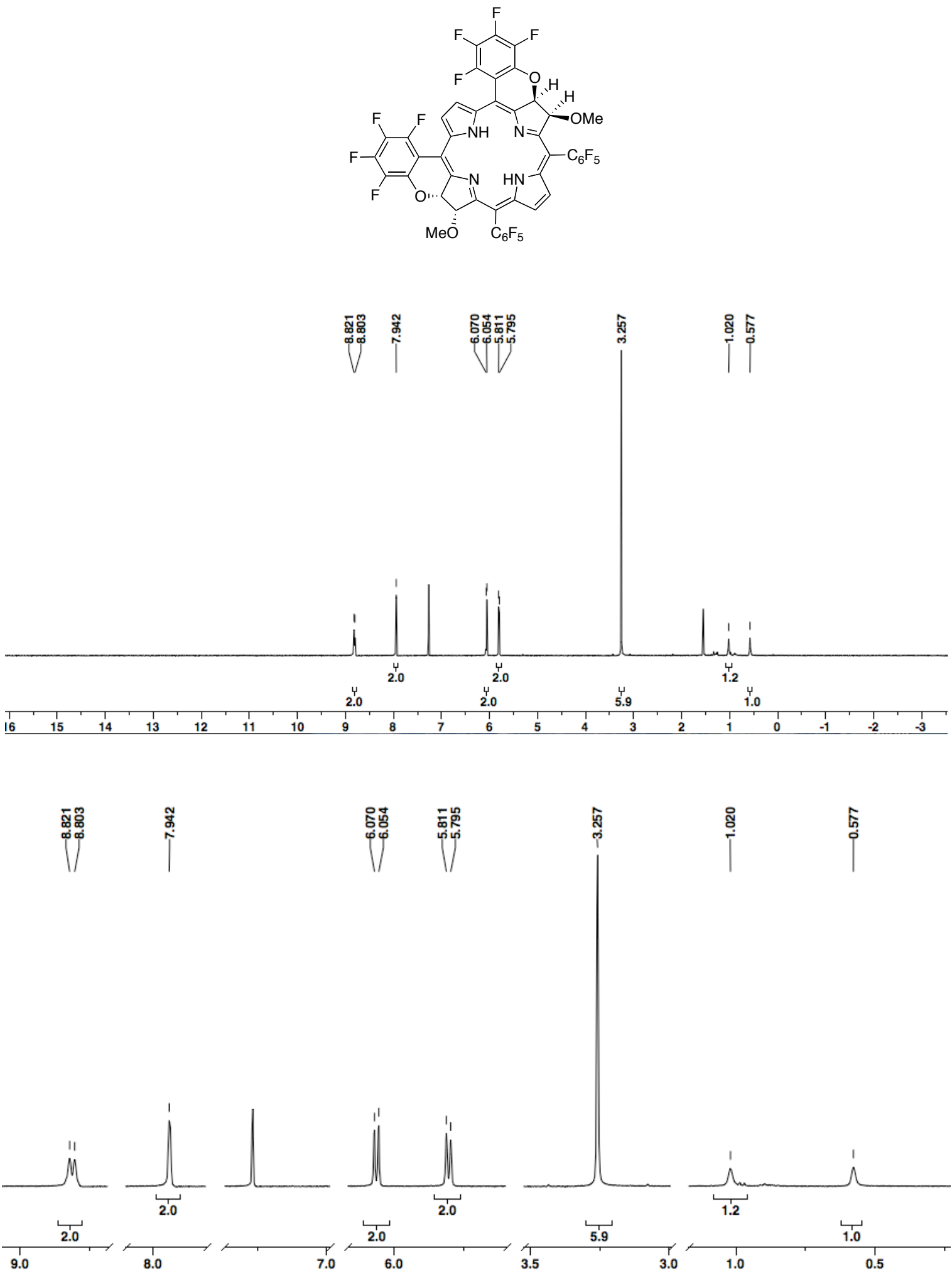

Figure S48. ${ }^{1} \mathrm{H}$ NMR spectrum $\left(400 \mathrm{MHz}, \mathrm{CDCl}_{3}\right)$ of $\mathbf{1 1}{ }^{\mathrm{Me}}-\boldsymbol{E}-\boldsymbol{s y \boldsymbol { n }}$. 

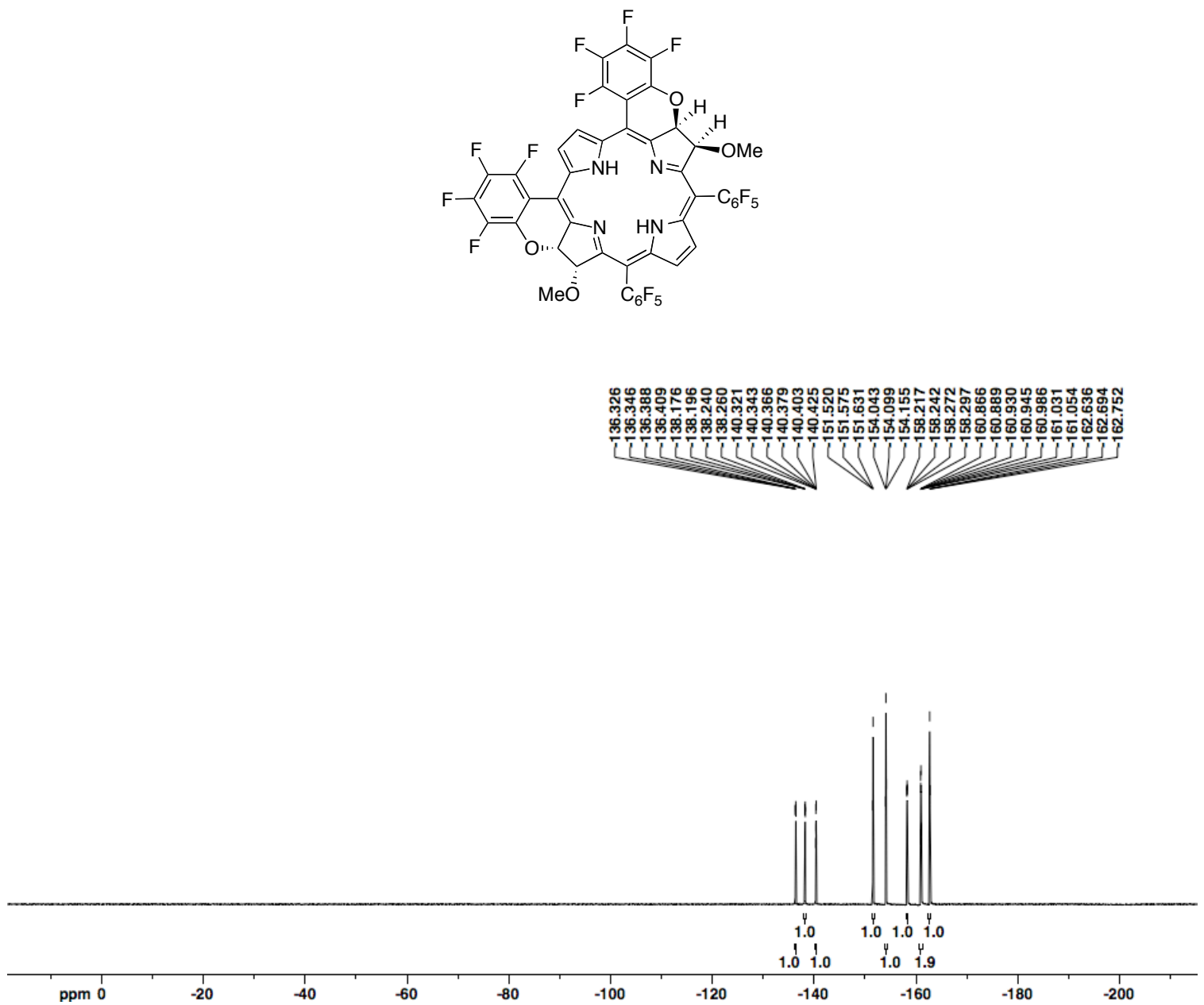

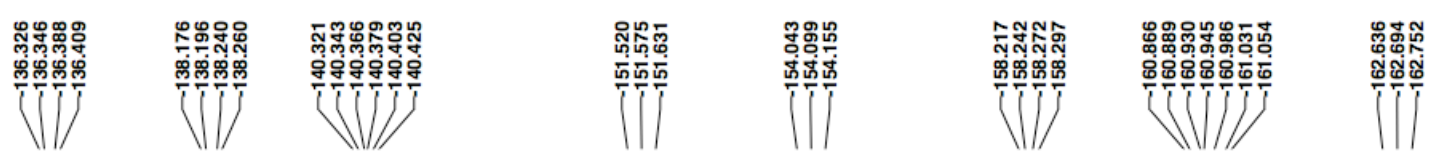

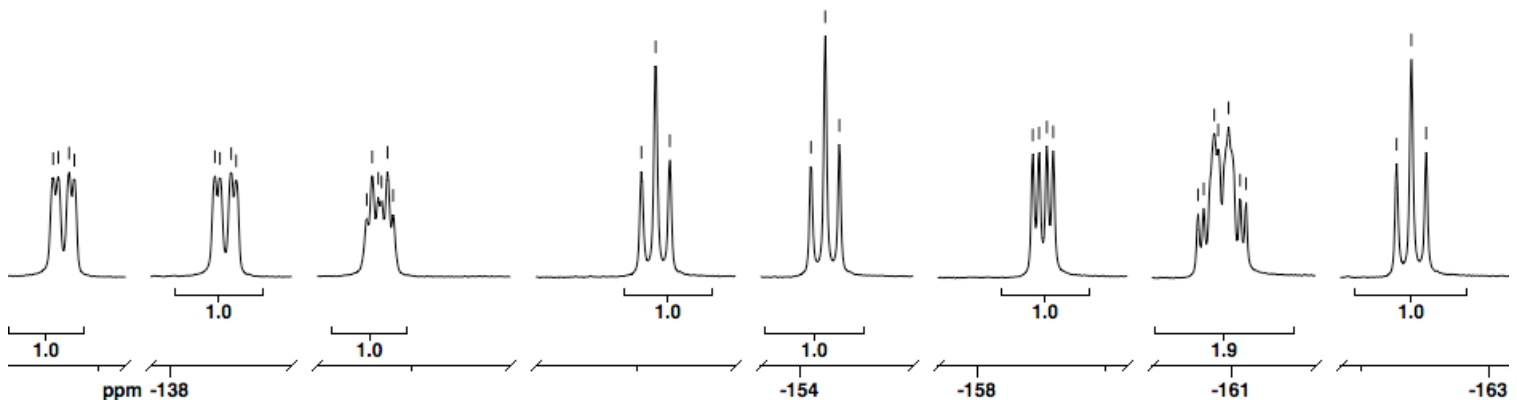

Figure S49. ${ }^{19} \mathrm{~F}$ NMR spectrum $\left(376 \mathrm{MHz}, \mathrm{CDCl}_{3}\right)$ of $\mathbf{1 1}^{\mathrm{Me}}-\boldsymbol{E}$-syn . 

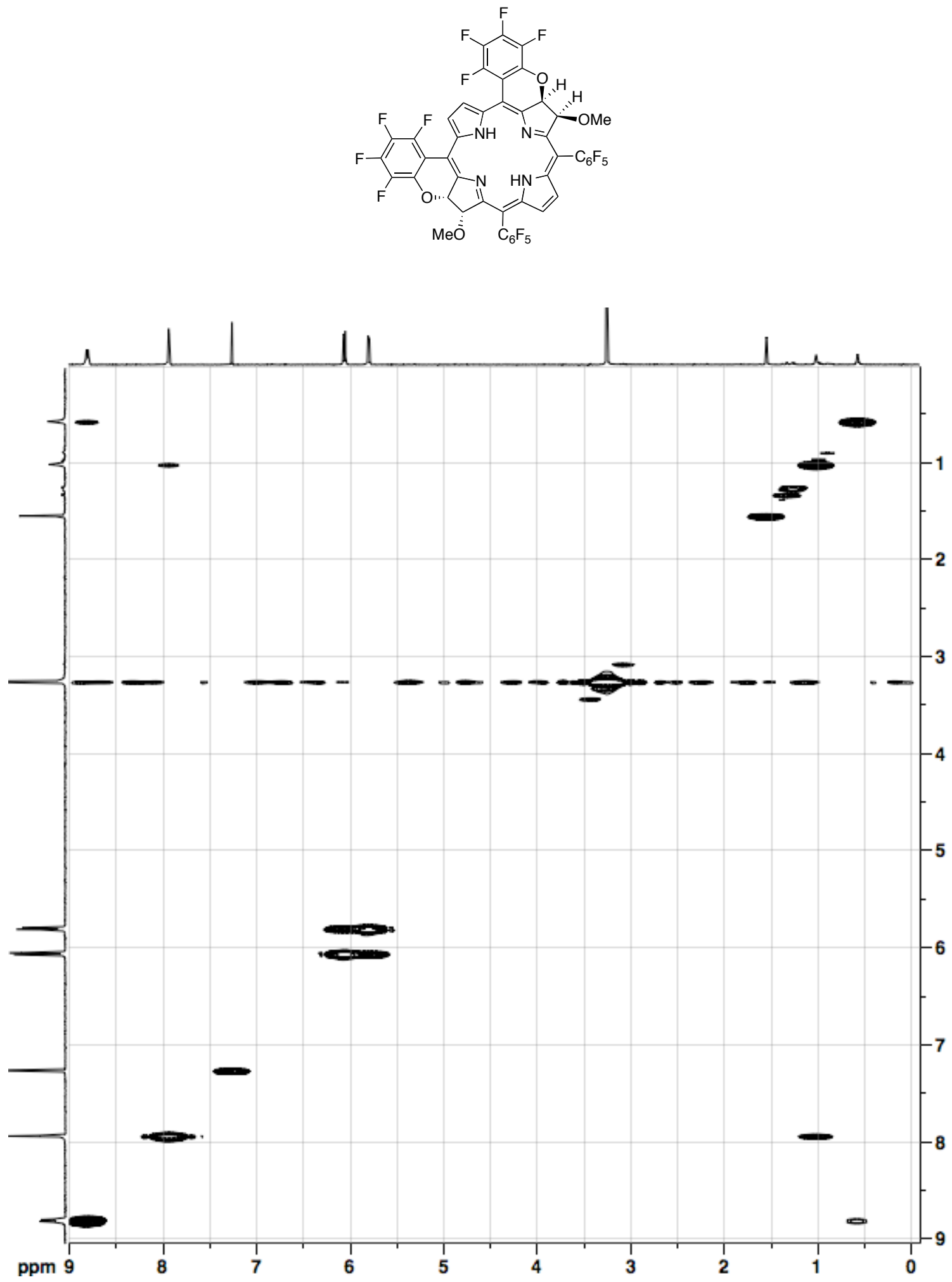

Figure S50. ${ }^{1} \mathrm{H},{ }^{1} \mathrm{H}-\mathrm{COSY}$ NMR spectrum $\left(400 \mathrm{MHz}, \mathrm{CDCl}_{3}\right)$ of $\mathbf{1 1}^{\mathbf{M e}}-\boldsymbol{E}$-syn . 

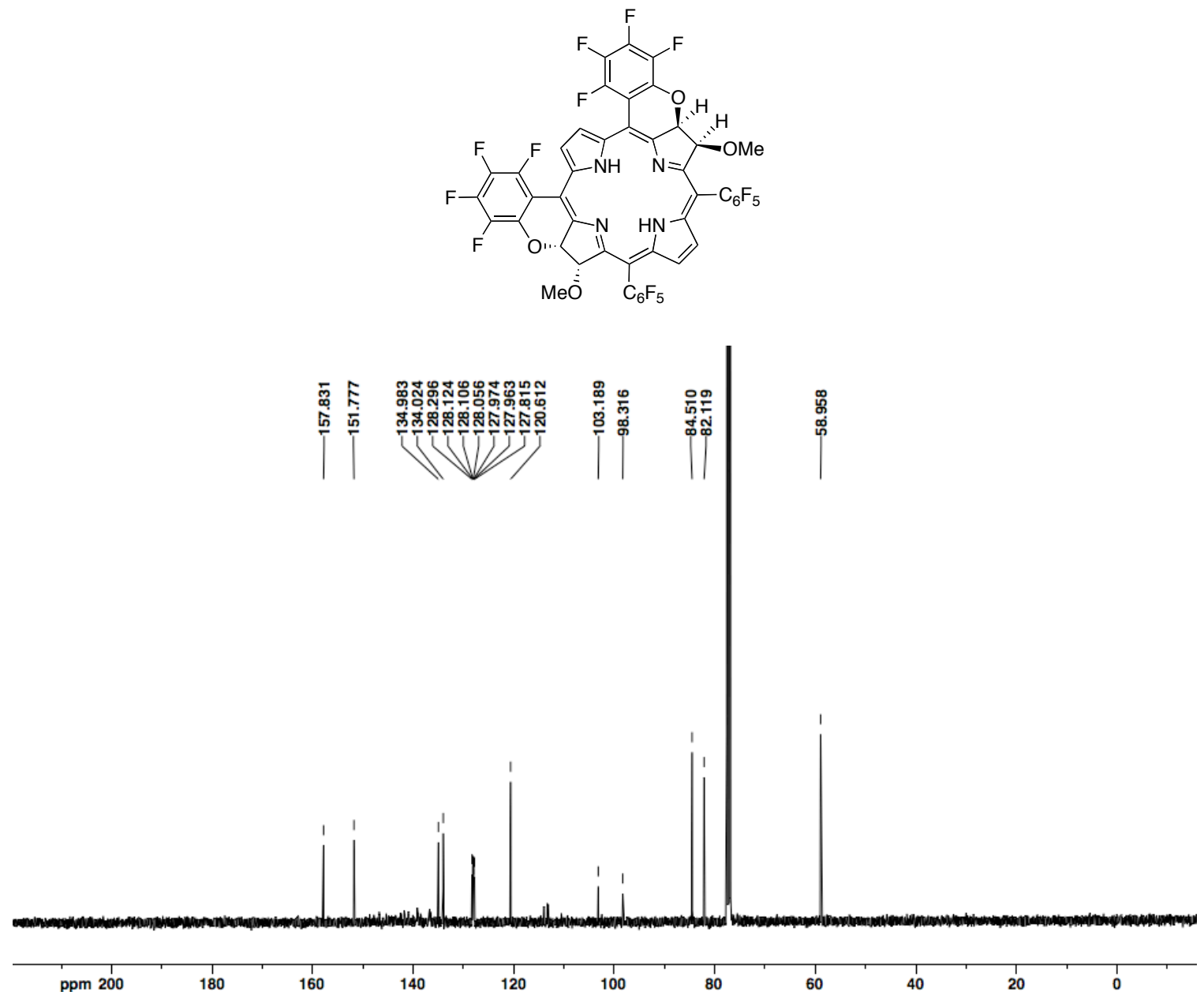

Figure S51. ${ }^{13} \mathrm{C}$ NMR spectrum $\left(100 \mathrm{MHz}, \mathrm{CDCl}_{3}\right)$ of $\mathbf{1 1}^{\mathrm{Me}}-\boldsymbol{E}$-syn. 

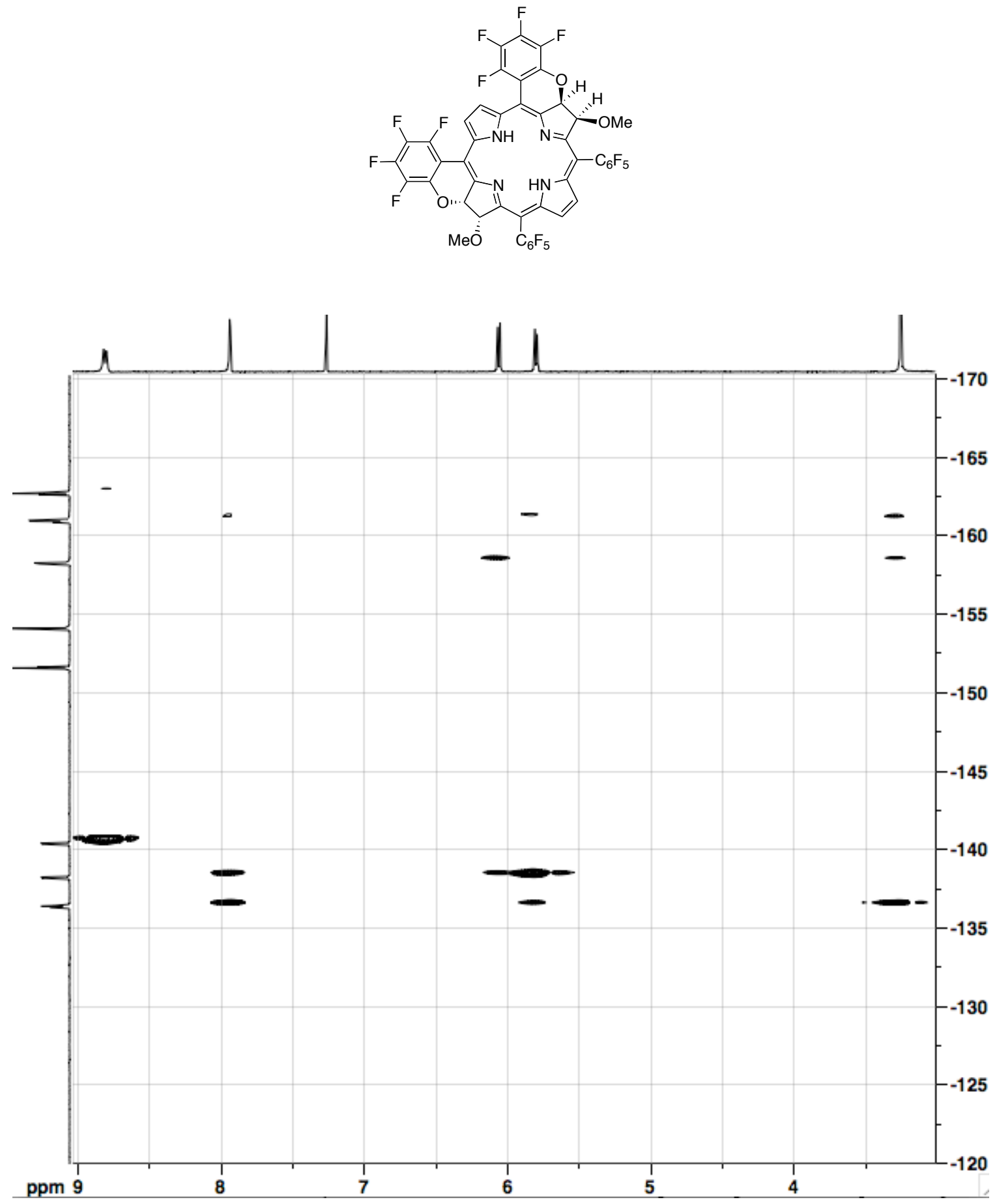

Figure S52. ${ }^{1} \mathrm{H},{ }^{19} \mathrm{~F}-\mathrm{HOESY}$ NMR spectrum $\left(400,376 \mathrm{MHz}, \mathrm{CDCl}_{3}\right)$ of $\mathbf{1 1}^{\mathrm{Me}}-\boldsymbol{E}$-syn. 

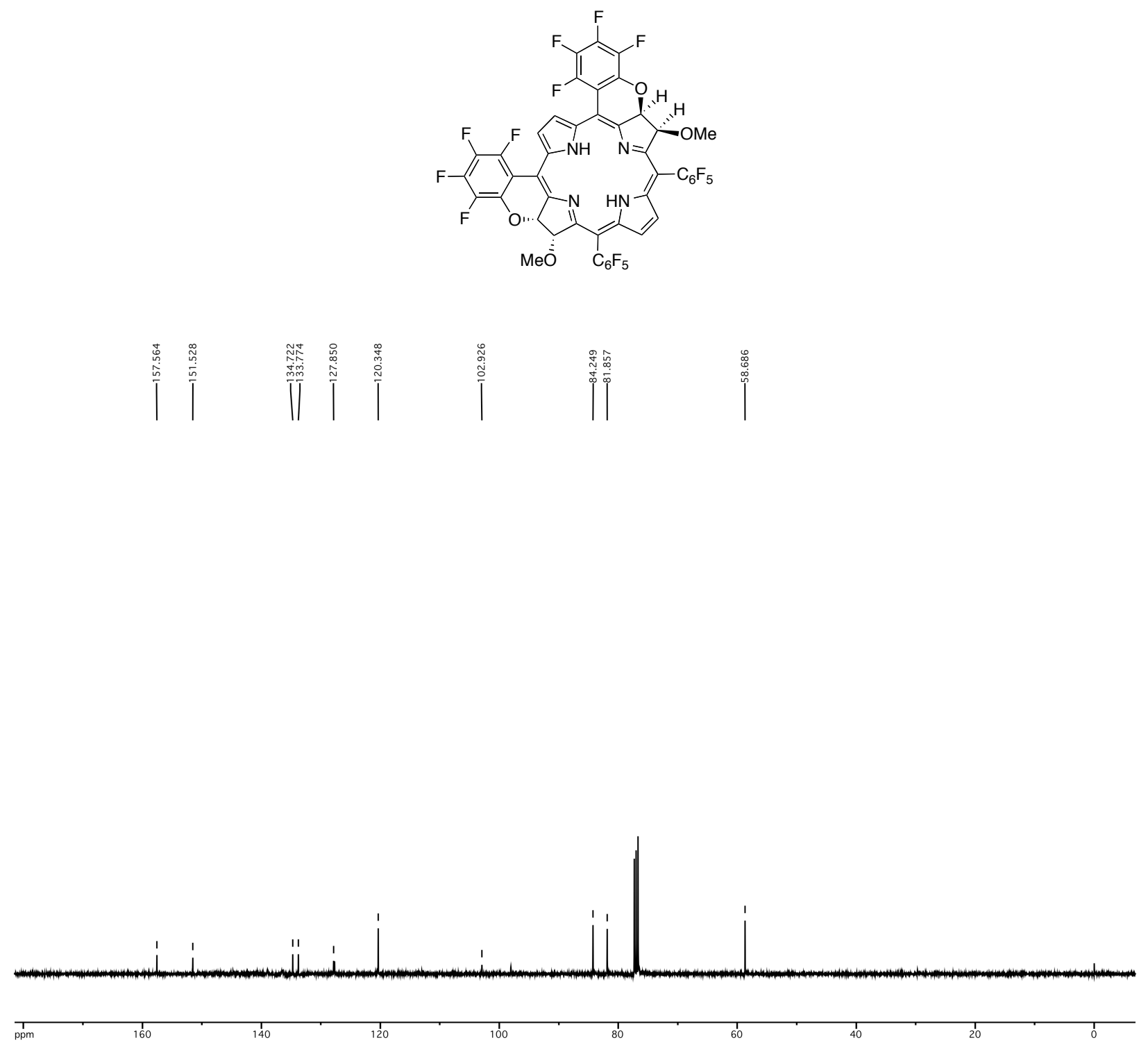

Figure S53. ${ }^{13} \mathrm{C}$ NMR spectrum $\left(100 \mathrm{MHz}, \mathrm{CDCl}_{3}\right)$ of $\mathbf{1 1}^{\mathrm{Me}}-\boldsymbol{E}$-syn. 

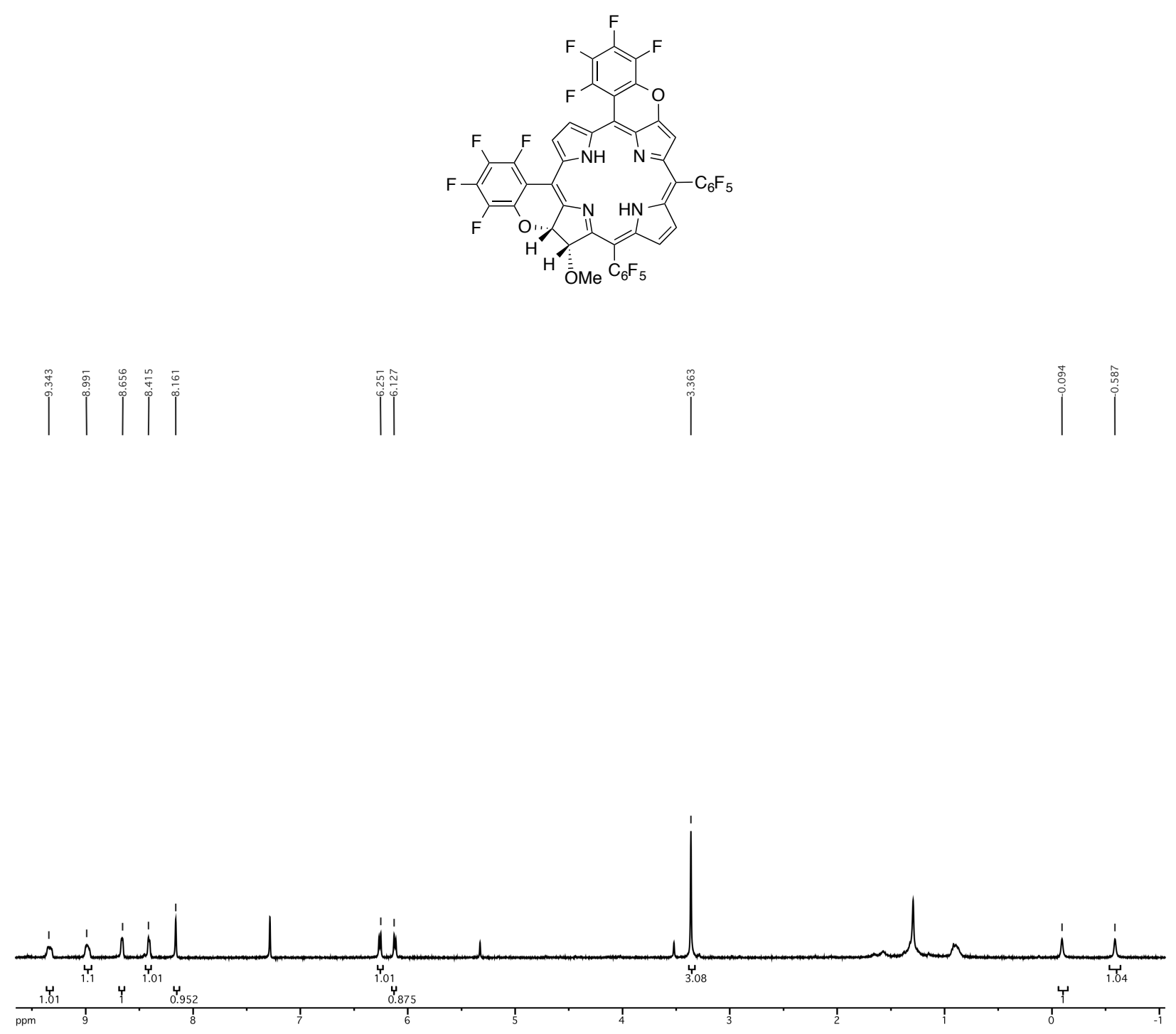

Figure S54. ${ }^{1} \mathrm{H}$ NMR spectrum $\left(400 \mathrm{MHz}, \mathrm{CDCl}_{3}\right)$ of 14-syn. 

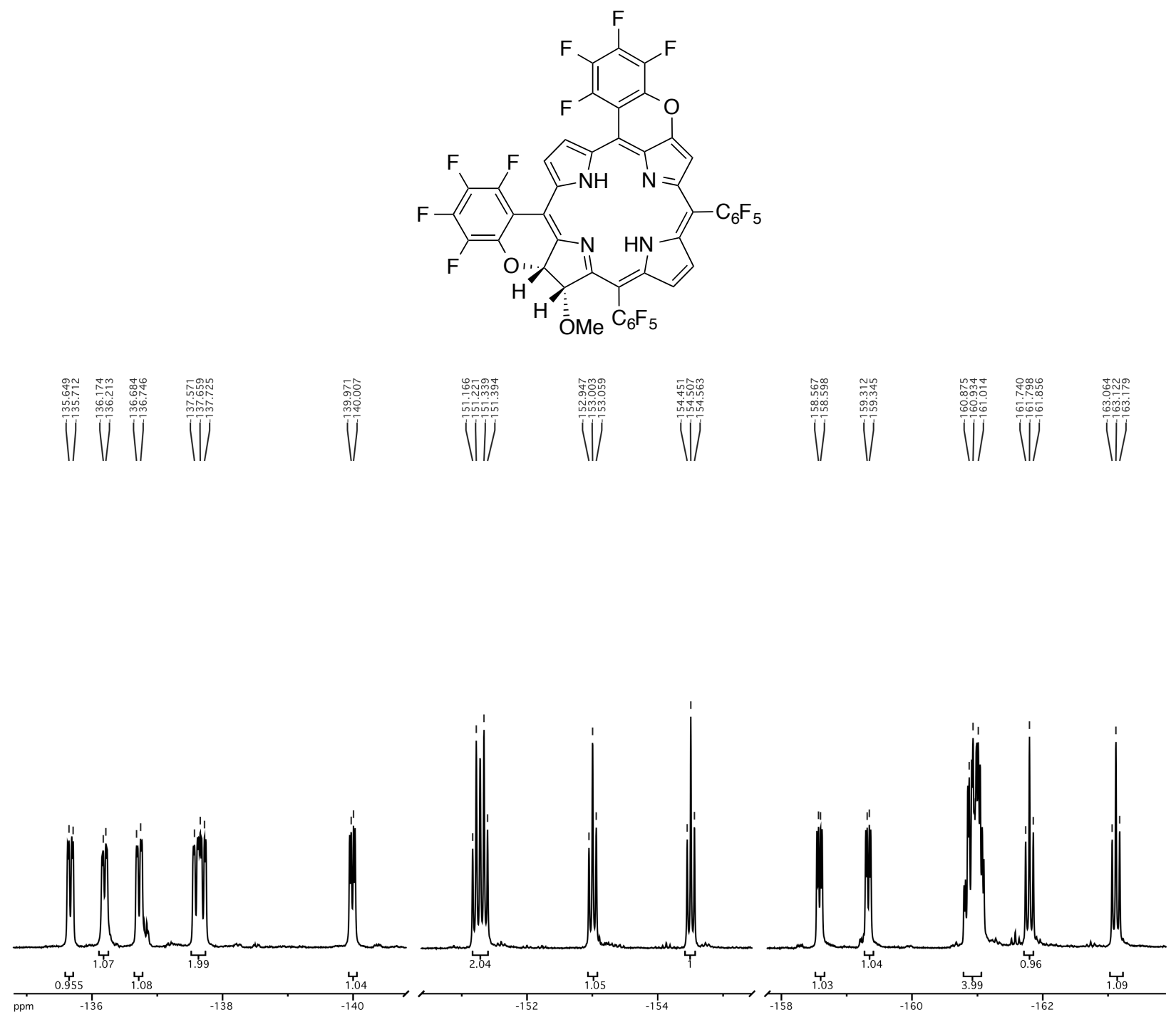

Figure S55. ${ }^{19} \mathrm{~F}$ NMR spectrum $\left(376 \mathrm{MHz}, \mathrm{CDCl}_{3}\right)$ of 14-syn.
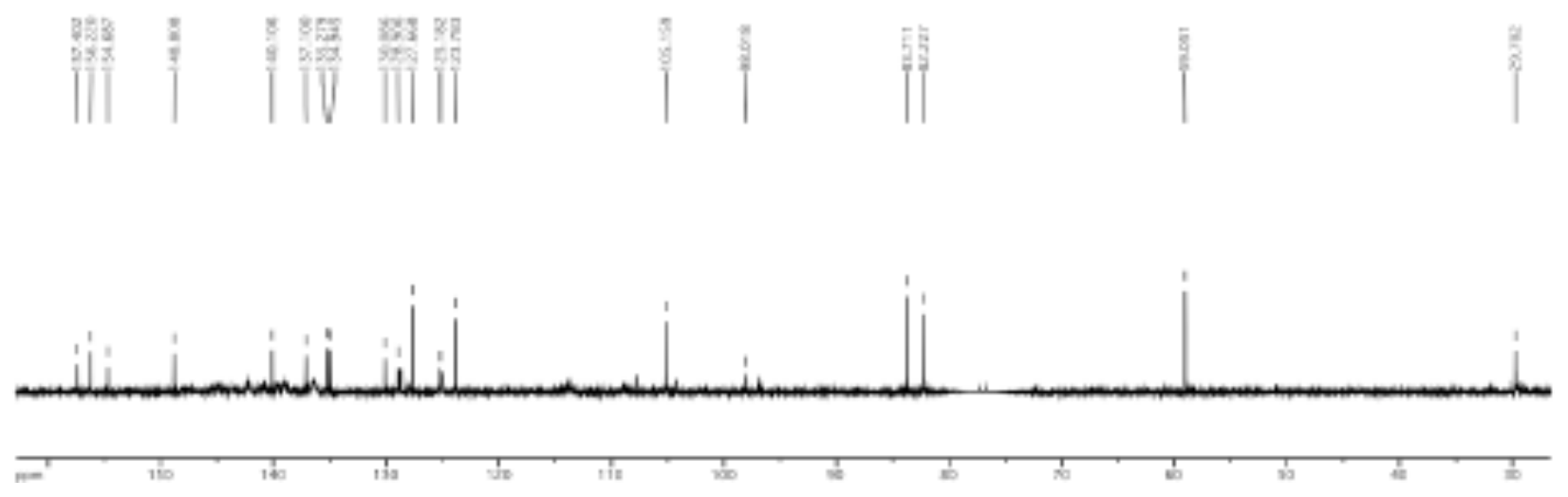

Figure S56. ${ }^{13} \mathrm{C}$ NMR spectrum $\left(100 \mathrm{MHz}, \mathrm{CDCl}_{3}\right)$ of 14-syn. 


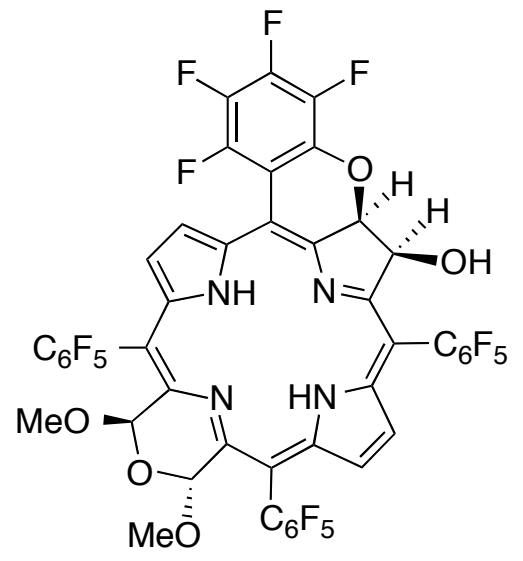

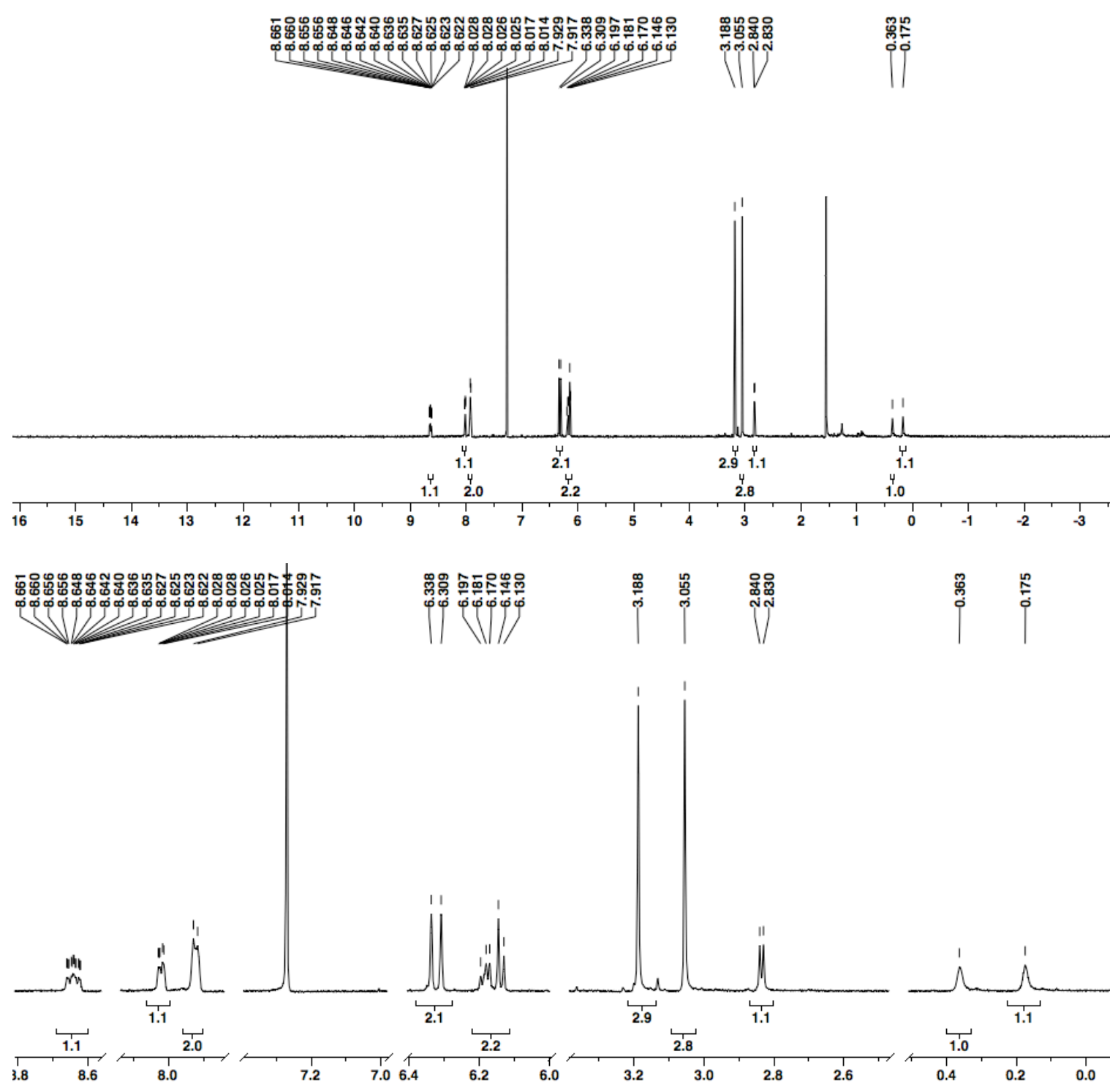

Figure S57. ${ }^{1} \mathrm{H}$ NMR spectrum $\left(400 \mathrm{MHz}, \mathrm{CDCl}_{3}\right)$ of $\mathbf{1 5}^{\mathrm{Me}}$. 


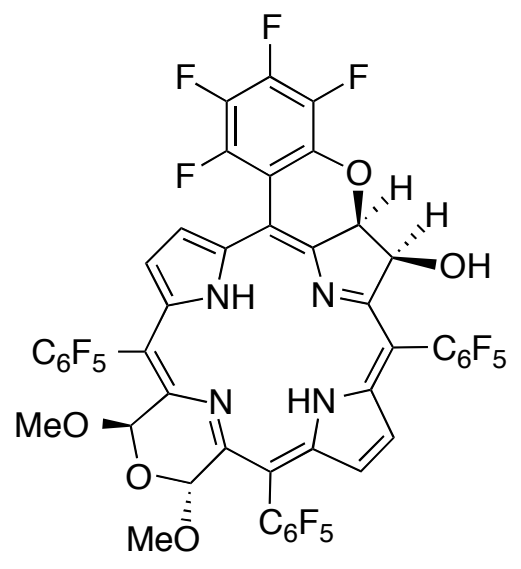
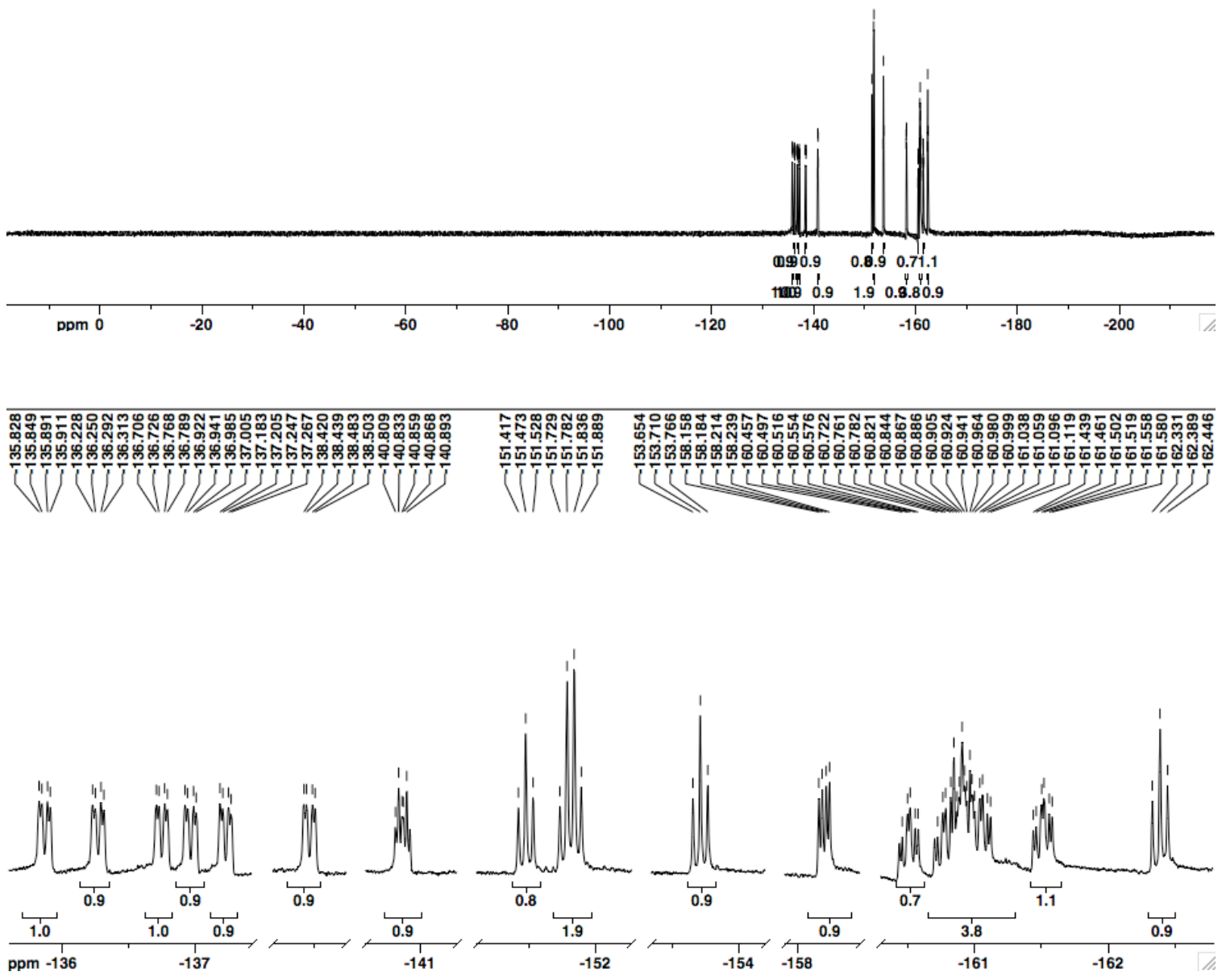

Figure S58. ${ }^{19} \mathrm{~F}$ NMR spectrum $\left(376 \mathrm{MHz}, \mathrm{CDCl}_{3}\right)$ of $\mathbf{1 5}^{\mathrm{Me}}$. 


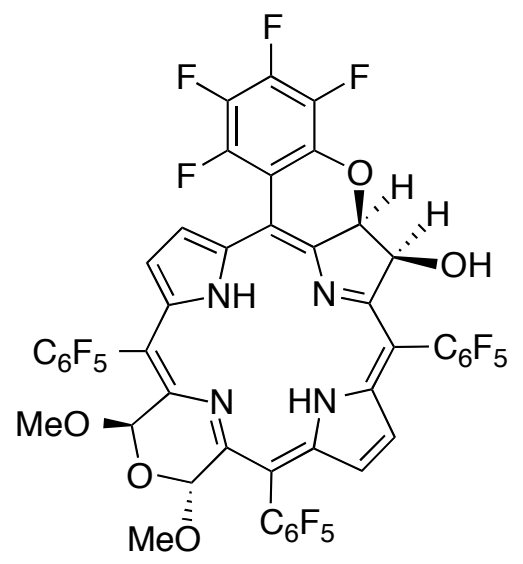

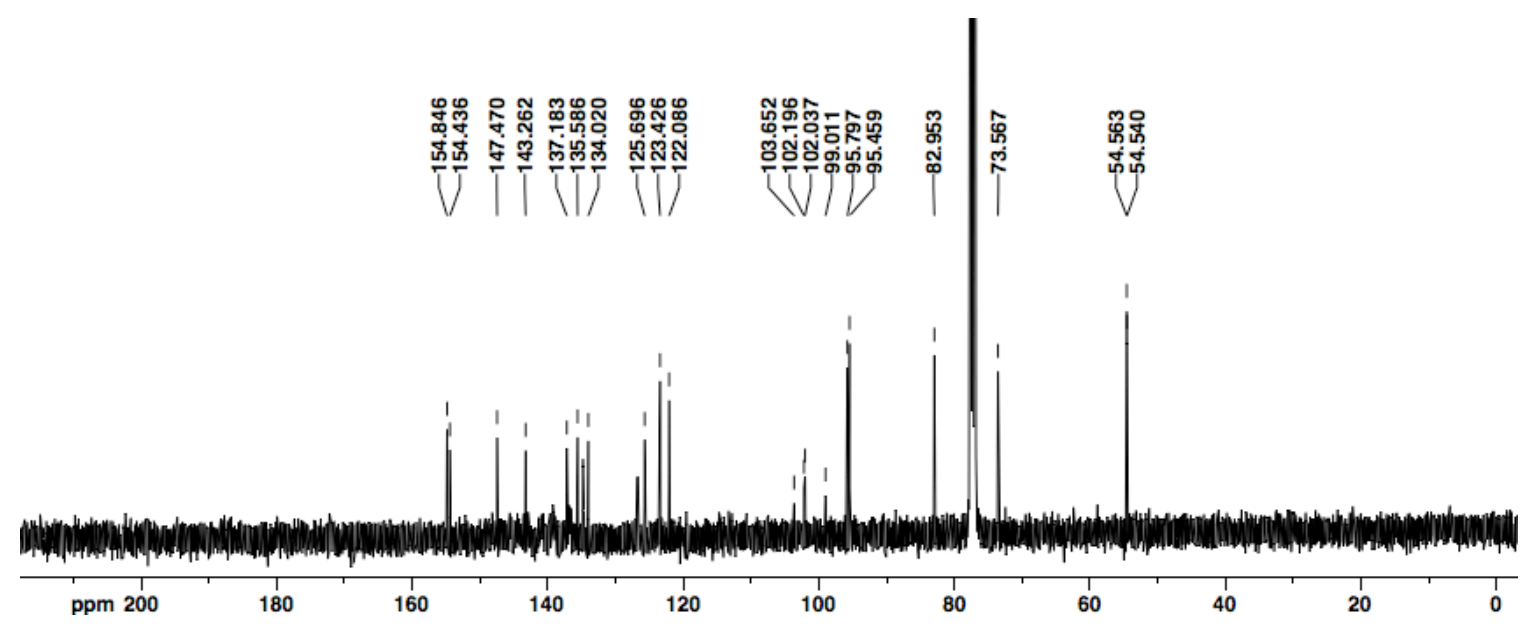

Figure S59. ${ }^{13} \mathrm{C}$ NMR spectrum $\left(100 \mathrm{MHz}, \mathrm{CDCl}_{3}\right)$ of $\mathbf{1 5}^{\mathrm{Me}}$. 

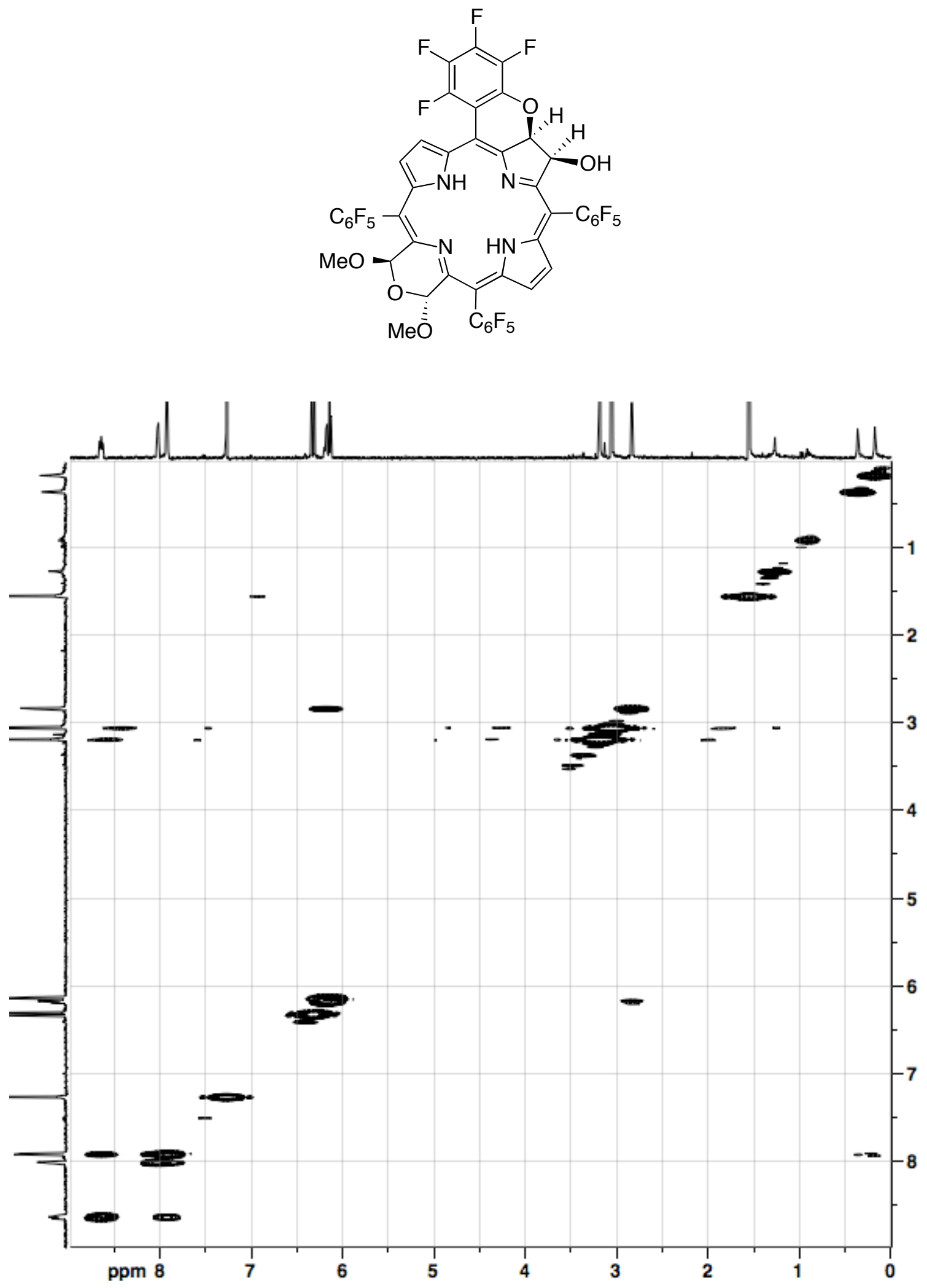

Figure S60. ${ }^{1} \mathrm{H},{ }^{1} \mathrm{H}-\mathrm{COSY}$ NMR spectrum $\left(400 \mathrm{MHz}, \mathrm{CDCl}_{3}\right)$ of $\mathbf{1 5}^{\mathrm{Me}}$. 


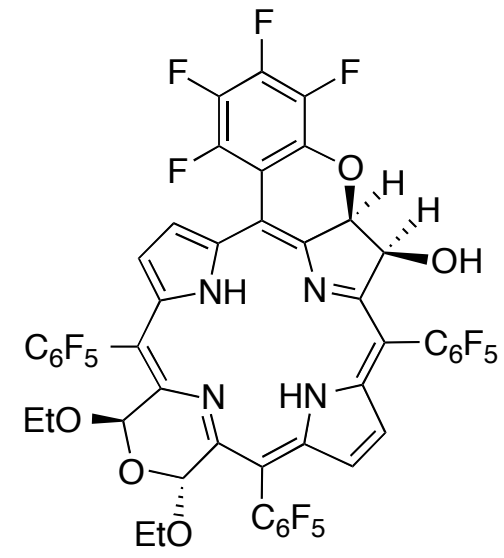

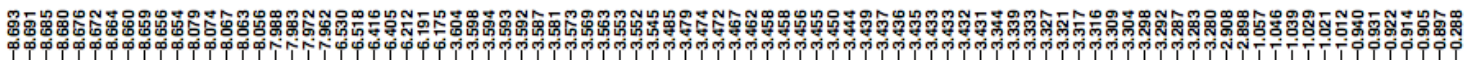

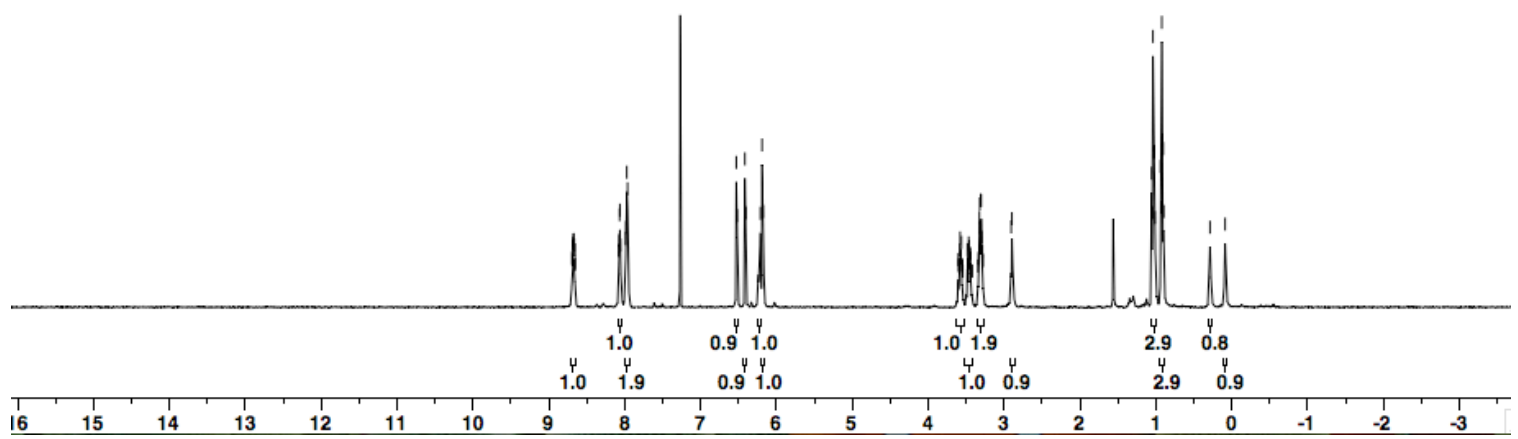

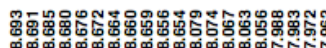
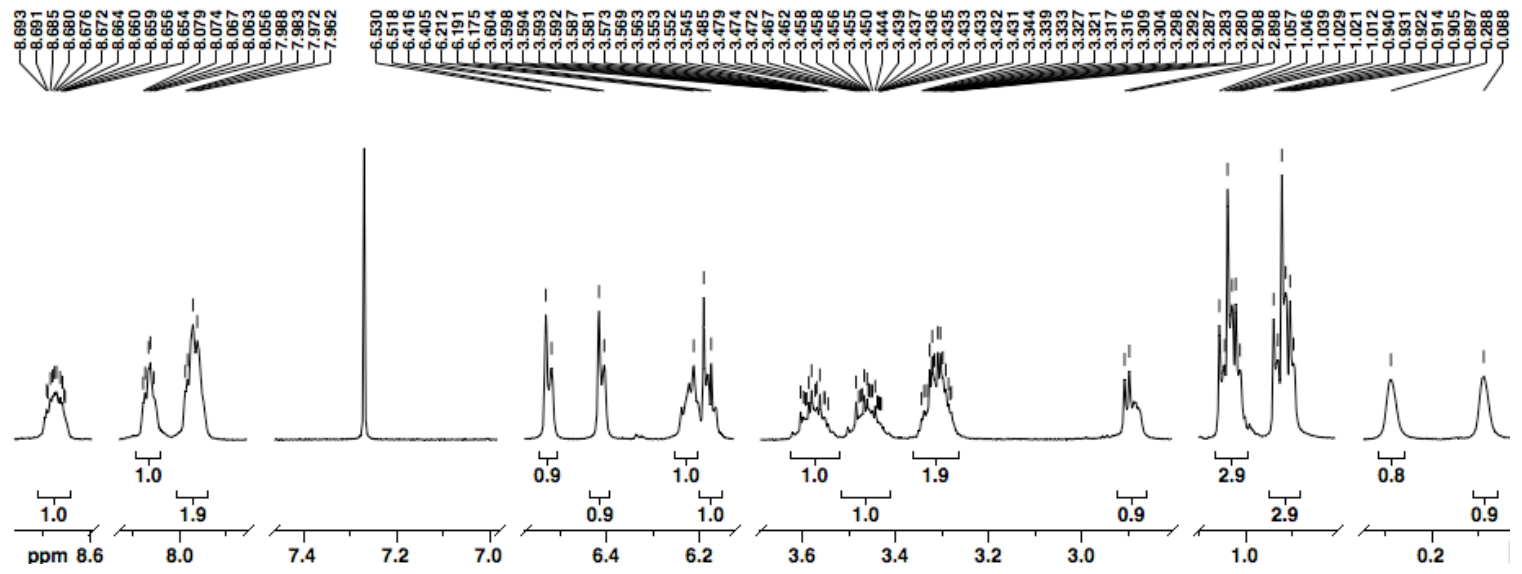

Figure S61. ${ }^{1} \mathrm{H}$ NMR spectrum $\left(400 \mathrm{MHz}, \mathrm{CDCl}_{3}\right)$ of $\mathbf{1 5}^{\mathbf{E t}}$. 


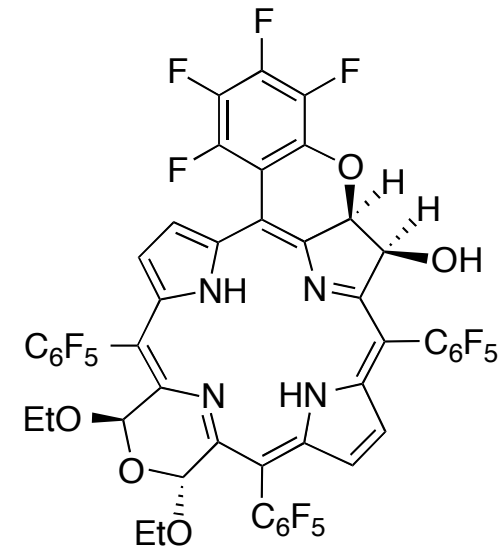

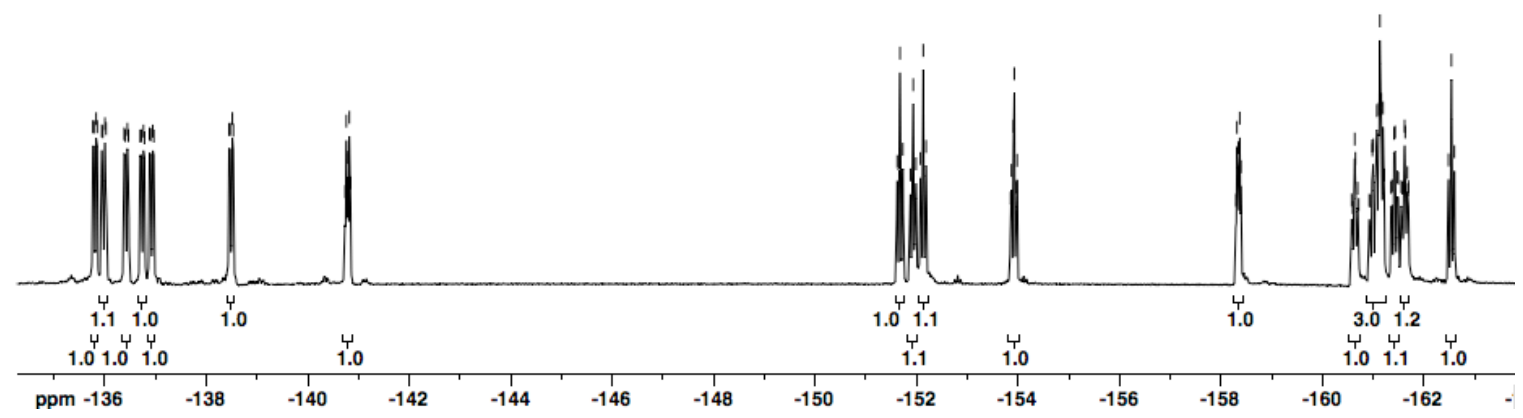

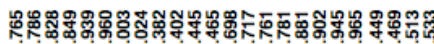

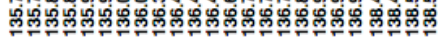

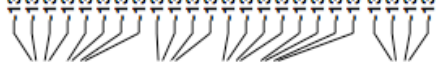

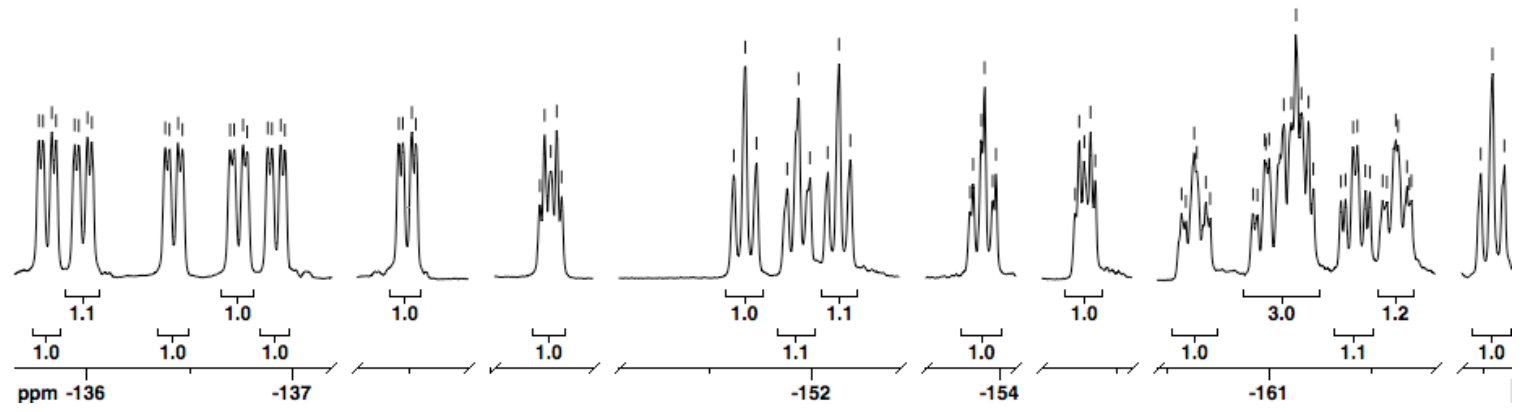

Figure S62. ${ }^{19} \mathrm{~F}$ NMR spectrum $\left(376 \mathrm{MHz}, \mathrm{CDCl}_{3}\right)$ of $\mathbf{1 5}^{\mathbf{E t}}$. 

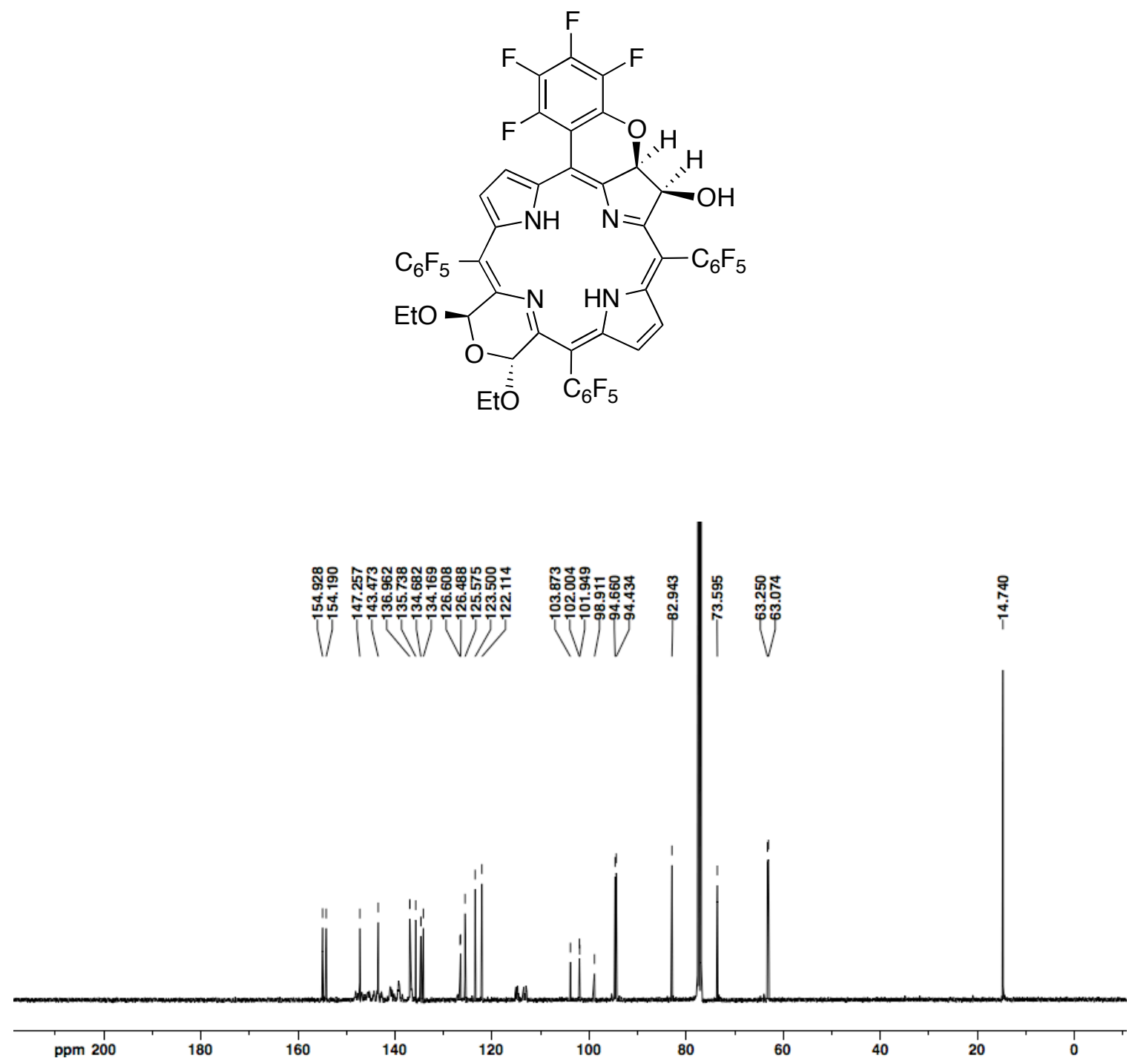

Figure S63. ${ }^{13} \mathrm{C}$ NMR spectrum $\left(100 \mathrm{MHz}, \mathrm{CDCl}_{3}\right)$ of $\mathbf{1 5}^{\mathbf{E t}}$. 


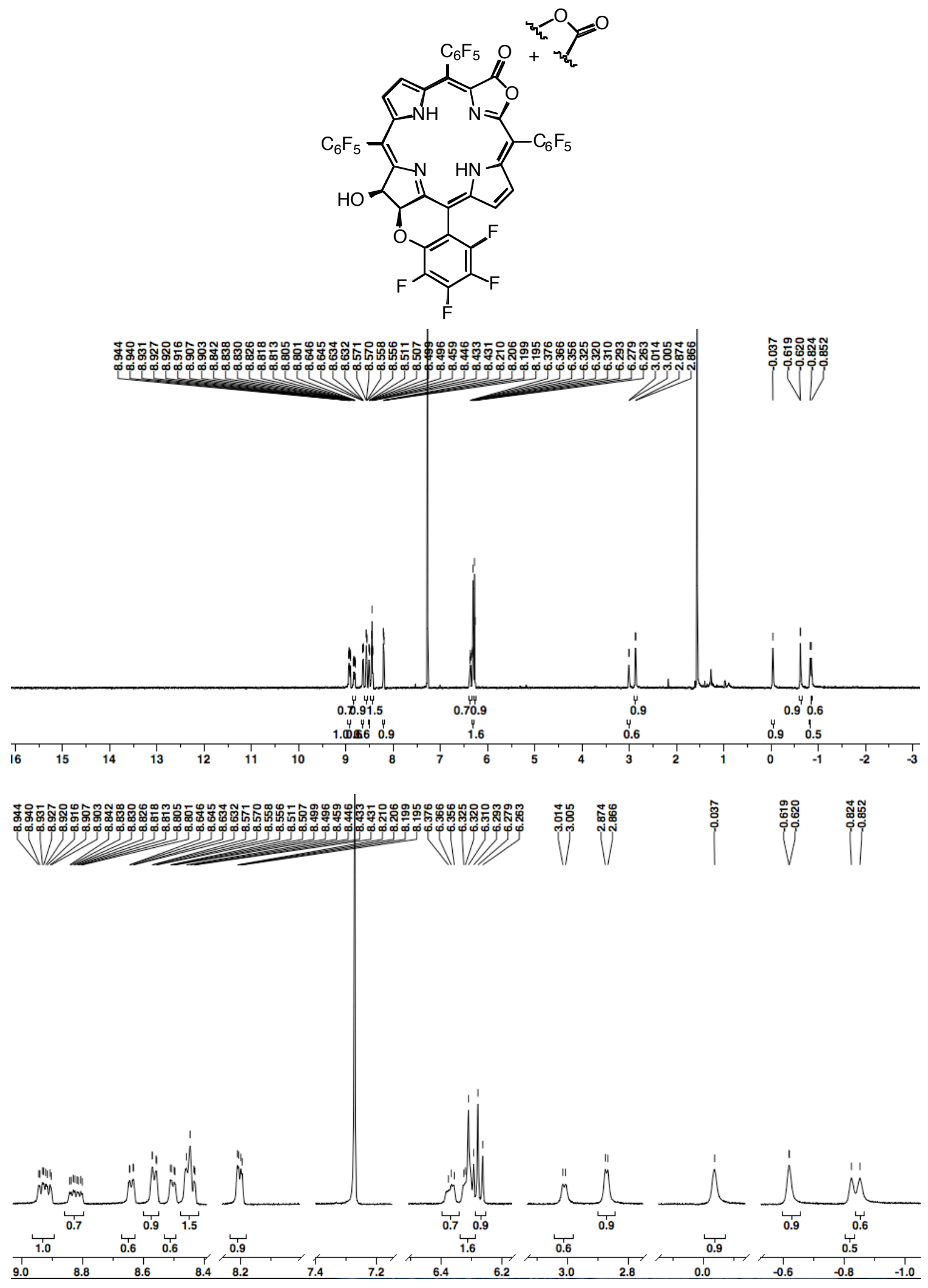

Figure S64. ${ }^{1} \mathrm{H}$ NMR spectrum $\left(400 \mathrm{MHz}, \mathrm{CDCl}_{3}\right)$ of $\mathbf{1 6 .}$ 

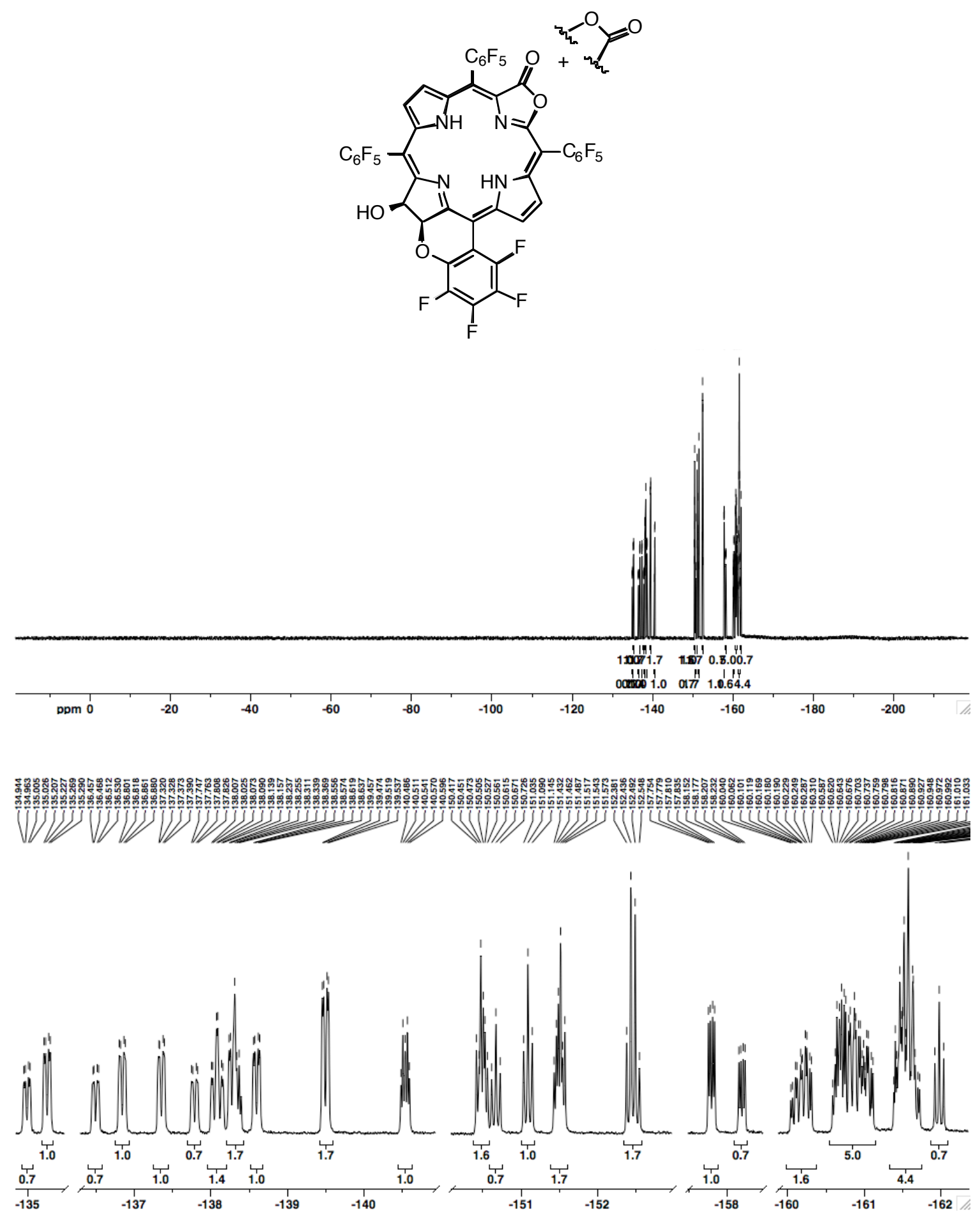

Figure S65. ${ }^{19} \mathrm{~F}$ NMR spectrum $\left(376 \mathrm{MHz}, \mathrm{CDCl}_{3}\right)$ of 16. 

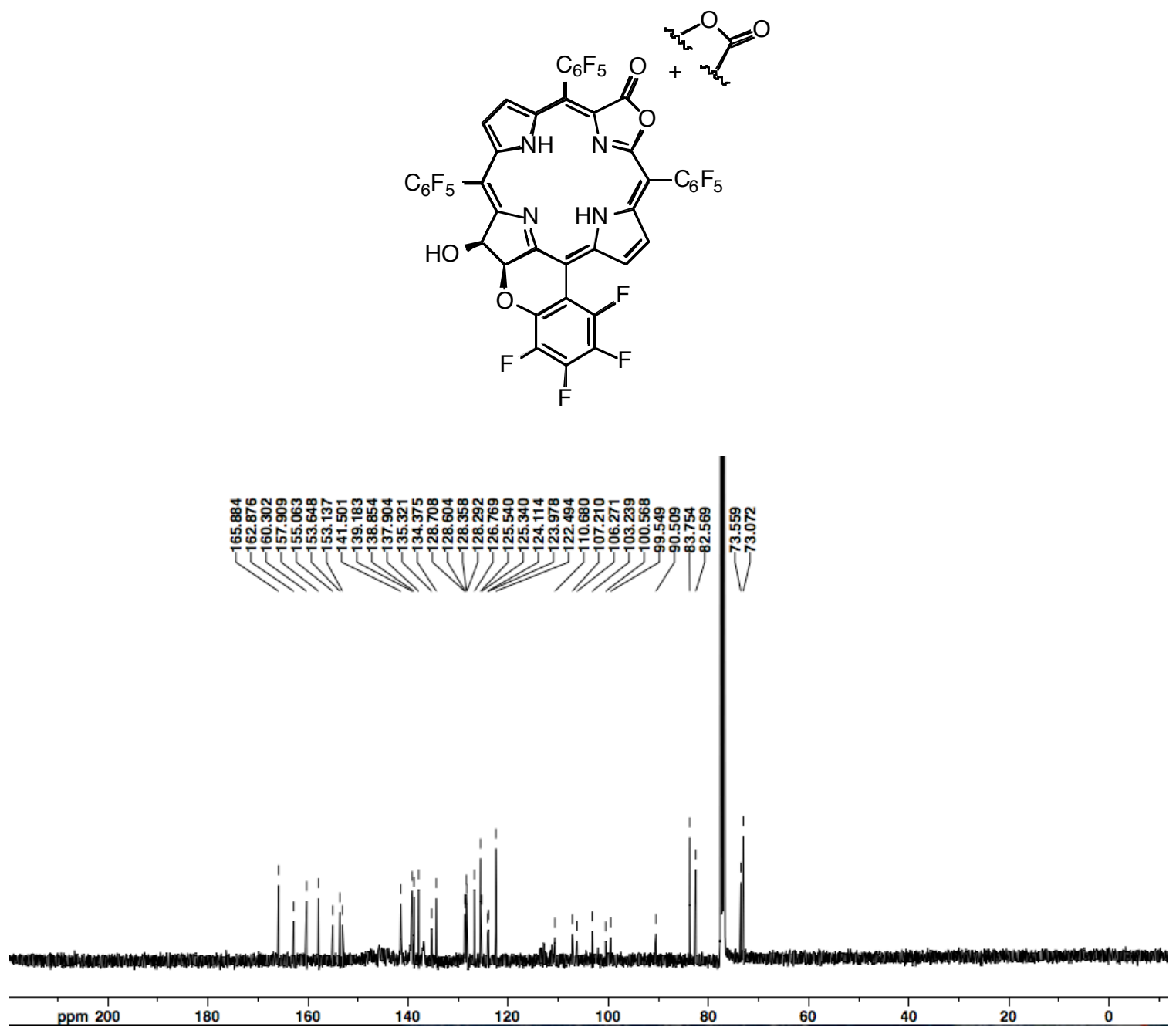

Figure S66. ${ }^{13} \mathrm{C} \mathrm{NMR}$ spectrum $\left(100 \mathrm{MHz}, \mathrm{CDCl}_{3}\right)$ of $\mathbf{1 6}$. 


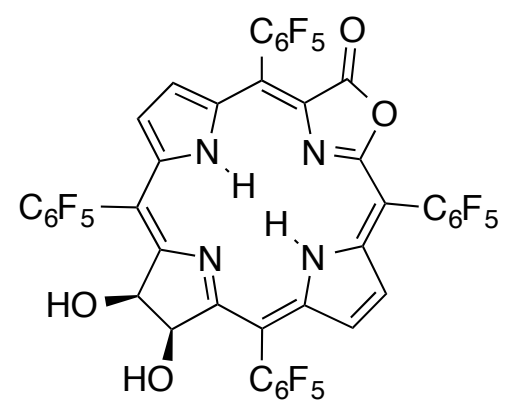
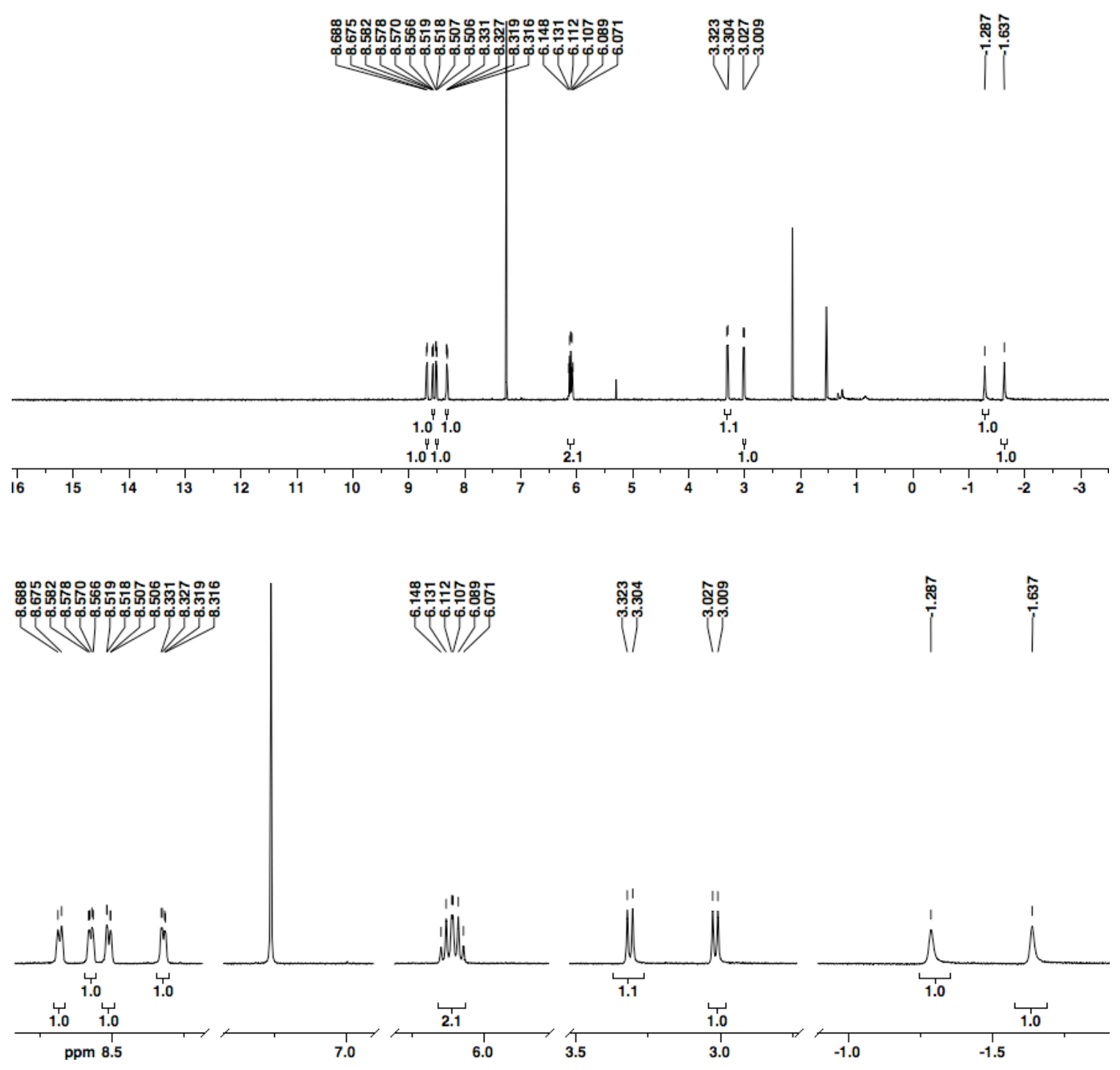

Figure S67. ${ }^{1} \mathrm{H}$ NMR spectrum $\left(400 \mathrm{MHz}, \mathrm{CDCl}_{3}\right)$ of 17. 


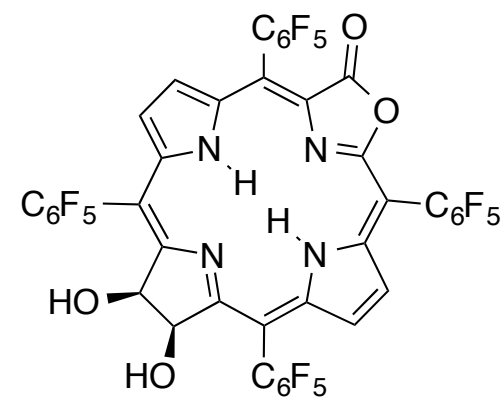
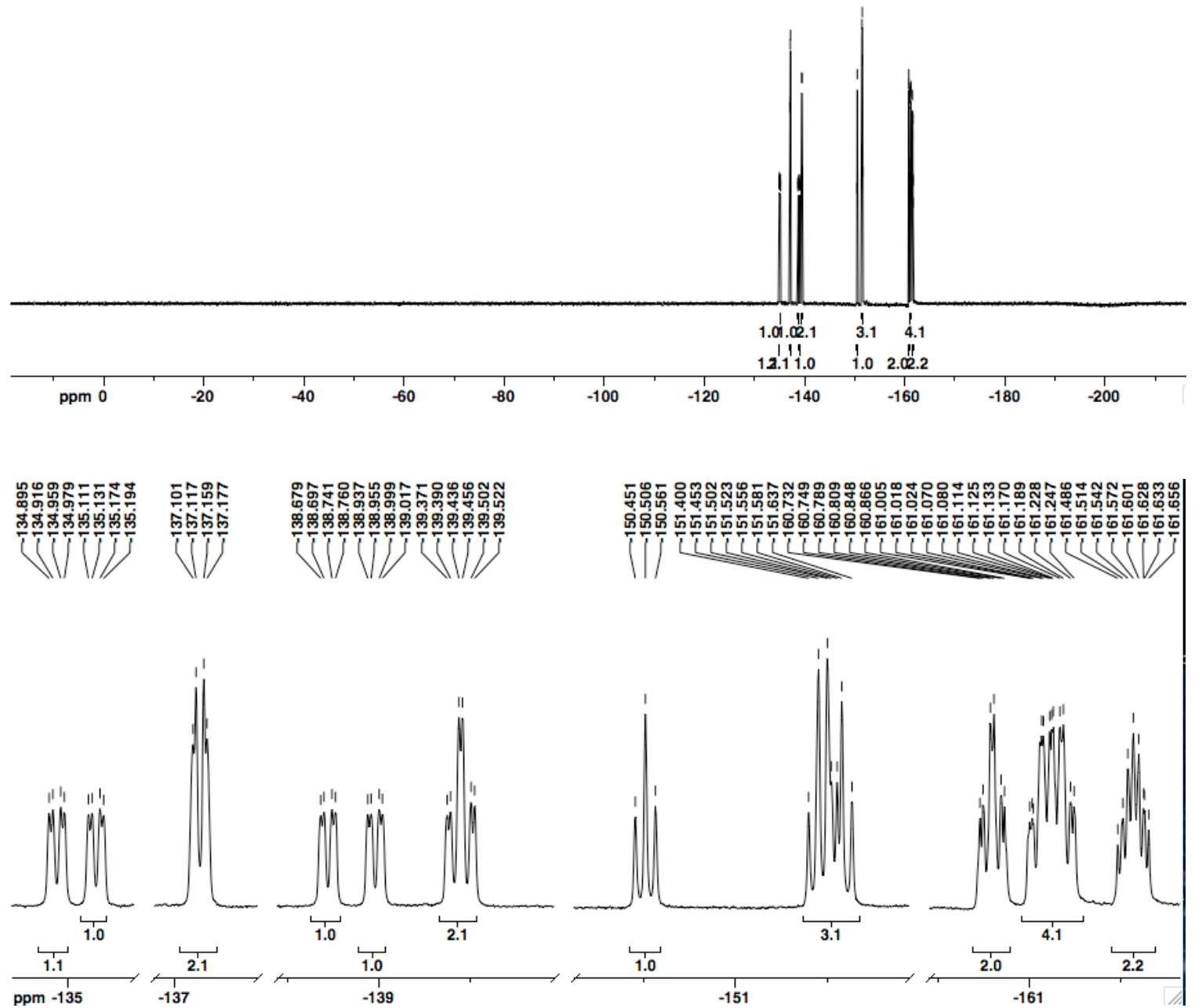

Figure S68. ${ }^{19} \mathrm{~F}$ NMR spectrum $\left(376 \mathrm{MHz}, \mathrm{CDCl}_{3}\right)$ of 17. 

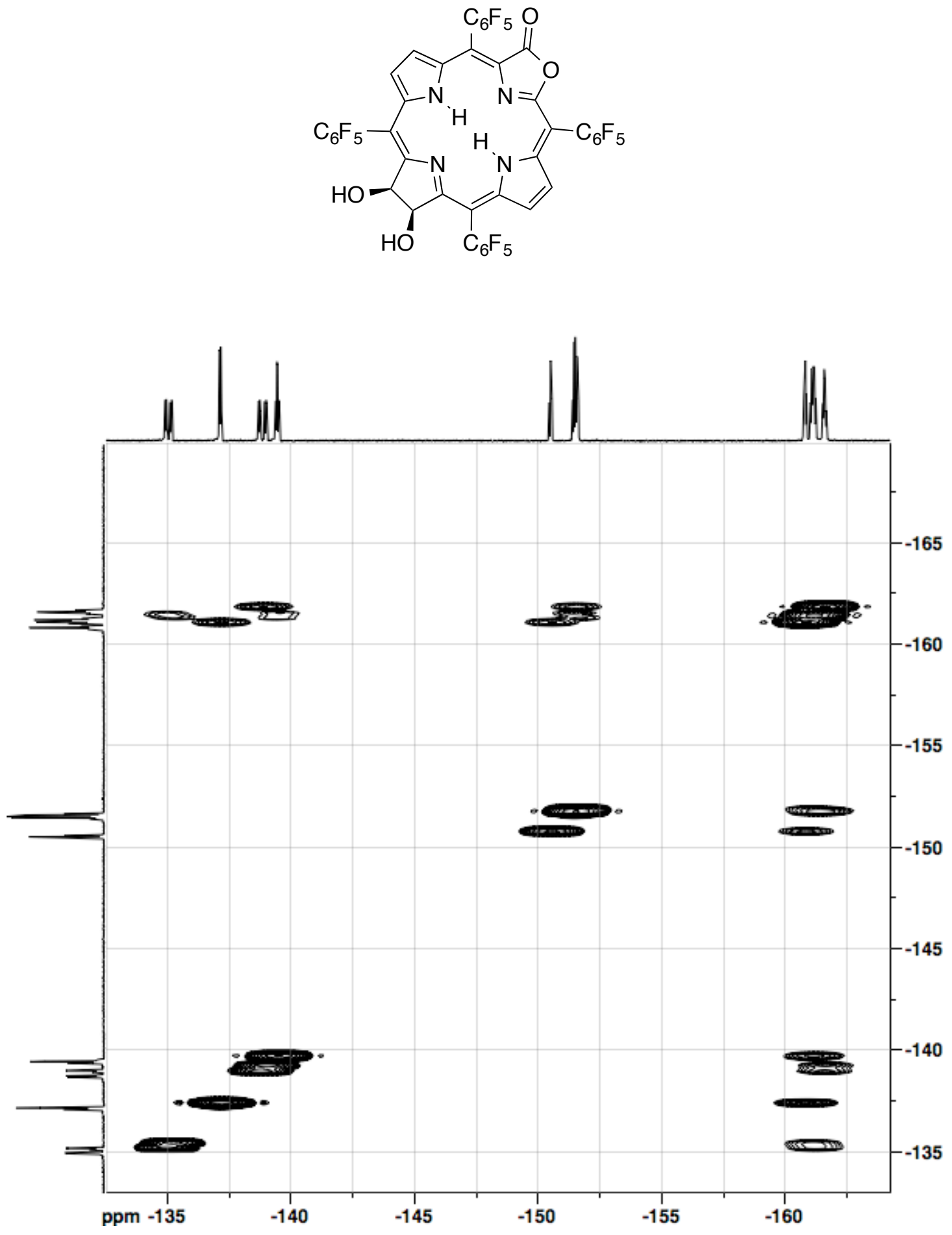

Figure S69. ${ }^{19} \mathrm{~F},{ }^{19} \mathrm{~F}-\mathrm{QF}-\mathrm{COSY}$ NMR spectrum $\left(376 \mathrm{MHz}, \mathrm{CDCl}_{3}\right)$ of 17. 


\section{Details to the X-ray Structure Analysis of 14-syn}

Single crystal XRD data for 14-syn were collected using a CCD four-circle diffractometer with Mo-K $\alpha$ radiation $(\lambda=0.71073 \AA)$. A single crystal was mounted on a micromesh mount using a trace of mineral oil and cooled in-situ to 100(2) K for data collection. Frames were collected, reflections were indexed and processed, and the files scaled and corrected for absorption using APEX2. ${ }^{1}$ The space group was assigned and the structure was solved by direct methods using XPREP within the SHELXTL suite of programs $^{2}$ and refined by full matrix least squares against $F^{2}$ with all reflections using Shelx $12013^{3}$ and the graphical interface Shelxle ${ }^{4} \mathrm{H}$ atoms attached to carbon and nitrogen atoms were positioned geometrically and constrained to ride on their parent atoms, with carbon hydrogen bond distances of $0.95 \AA$ for alkene and aromatic $\mathrm{C}-\mathrm{H}, 1.00$ and $0.98 \AA$ for aliphatic $\mathrm{C}-\mathrm{H}$ and $\mathrm{CH}_{3}$, and $0.88 \AA$ for $\mathrm{N}-\mathrm{H}$ moieties, respectively. $\mathrm{U}_{\text {iso }}(\mathrm{H})$ values were set to a multiple of $\mathrm{U}_{\mathrm{eq}}(\mathrm{C} / \mathrm{N})$ with 1.5 for $\mathrm{CH}_{3}$ and 1.2 for $\mathrm{C}-\mathrm{H}$ and $\mathrm{N}-\mathrm{H}$ units, respectively. A solvate pyridyl molecule is disordered around an inversion center and partially occupied. The molecule was constrained to resemble an ideal hexagon with $\mathrm{C}-\mathrm{C}$ and $\mathrm{C}-\mathrm{N}$ bond distances of $1.39 \AA$. Overlapping atoms were constrained to have identical ADPs and were restrained to be approximately isotropic. The partial occupancy is associated with disorder of a pentafluoro phenyl group. The minor orientation, present in the absence of the pyridyl molecule, was restrained to have a similar geometry to that of the major moiety, and to be flat within a standard deviation of $0.01 \AA$ squared. The ADPs of the minor moiety were restrained to be similar to those of their neighbors. Subject to these conditions, the final occupancy rates refined to 0.901(3) and 0.099(3). Reflections $001,010,110,011$ were obstructed by the beam stop and were omitted from the refinement. Complete crystallographic data, in CIF format, have been deposited with the Cambridge Crystallographic Data Centre. CCDC 1429883 contains the supplementary crystallographic data for this paper. These data can be obtained free of charge from The Cambridge Crystallographic Data Centre via www.ccdc.cam.ac.uk/data_request/cif.

\footnotetext{
${ }^{1}$ Apex2 v2014.1, Bruker AXS Inc.: Madison (WI), USA, 2009.

2 a) SHELXTL (Version 6.14) Bruker Advanced X-ray Solutions, Bruker AXS Inc., Madison, Wisconsin: USA. 2000-2003. b) Sheldrick, G. M. Acta Cryst. 2008, A64, 112-122.

3 a) Sheldrick, G. M. Acta Cryst. 2015, C71, 3-8. b) Sheldrick, G. M. (2013). University of Göttingen, Germany.

${ }^{4}$ Hübschle, C. B., Sheldrick, G. M. and Dittrich, B. J. Appl. Cryst., 2011, 44, 1281-1284.
} 
Table S1. Experimental details to the Crystal Structure Analysis of 14-syn

$$
\text { 14-syn }
$$

\section{Crystal data}

Chemical formula

$M_{\mathrm{r}}$

Crystal system, space group

Temperature $(\mathrm{K})$

$a, b, c(\AA)$

$\alpha, \beta, \gamma\left({ }^{\circ}\right)$

$V\left(\AA^{3}\right)$

$Z$

$F(000)$

$D_{x}\left(\mathrm{Mg} \mathrm{m}^{-3}\right)$

Radiation type

No. of reflections for cell measurement

$\theta$ range $\left(^{\circ}\right)$ for cell measurement

$\mu\left(\mathrm{mm}^{-1}\right)$

Crystal shape

Colour

Crystal size (mm)

\section{Data collection}

Diffractometer

Radiation source

Scan method

Absorption correction

$T_{\min }, T_{\max }$

No. of measured, independent and observed $[I>2 \sigma(I)]$ reflections

$R_{\text {int }}$

$\theta$ values $\left({ }^{\circ}\right)$

$(\sin \theta / \lambda)_{\max }\left(\AA^{-1}\right)$

Range of $h, k, l$

\section{Refinement}

$R\left[F^{2}>2 \sigma\left(F^{2}\right)\right], w R\left(F^{2}\right), S$

No. of reflections

No. of parameters

No. of restraints

$\mathrm{H}$-atom treatment

$\Delta \rho_{\max }, \Delta \rho_{\min }\left(\mathrm{e} \AA^{-3}\right)$

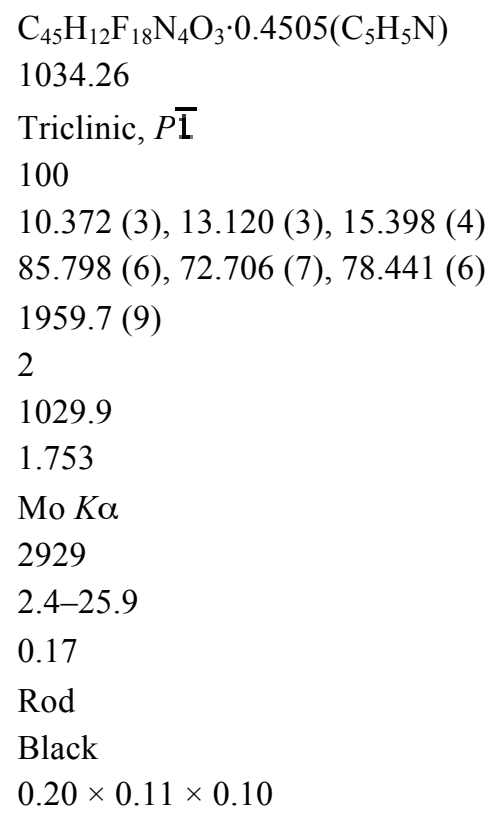

Computer programs: Apex2 v2012.4-3 (Bruker, 2012), SAINT V8.18C (Bruker, 2012), SHELXS97 (Sheldrick, 2008), SHELXL2013 (Sheldrick, 2013), SHELXLE Rev609 (Hübschle et al., 2011), SHELXTL (Bruker, 2003). 


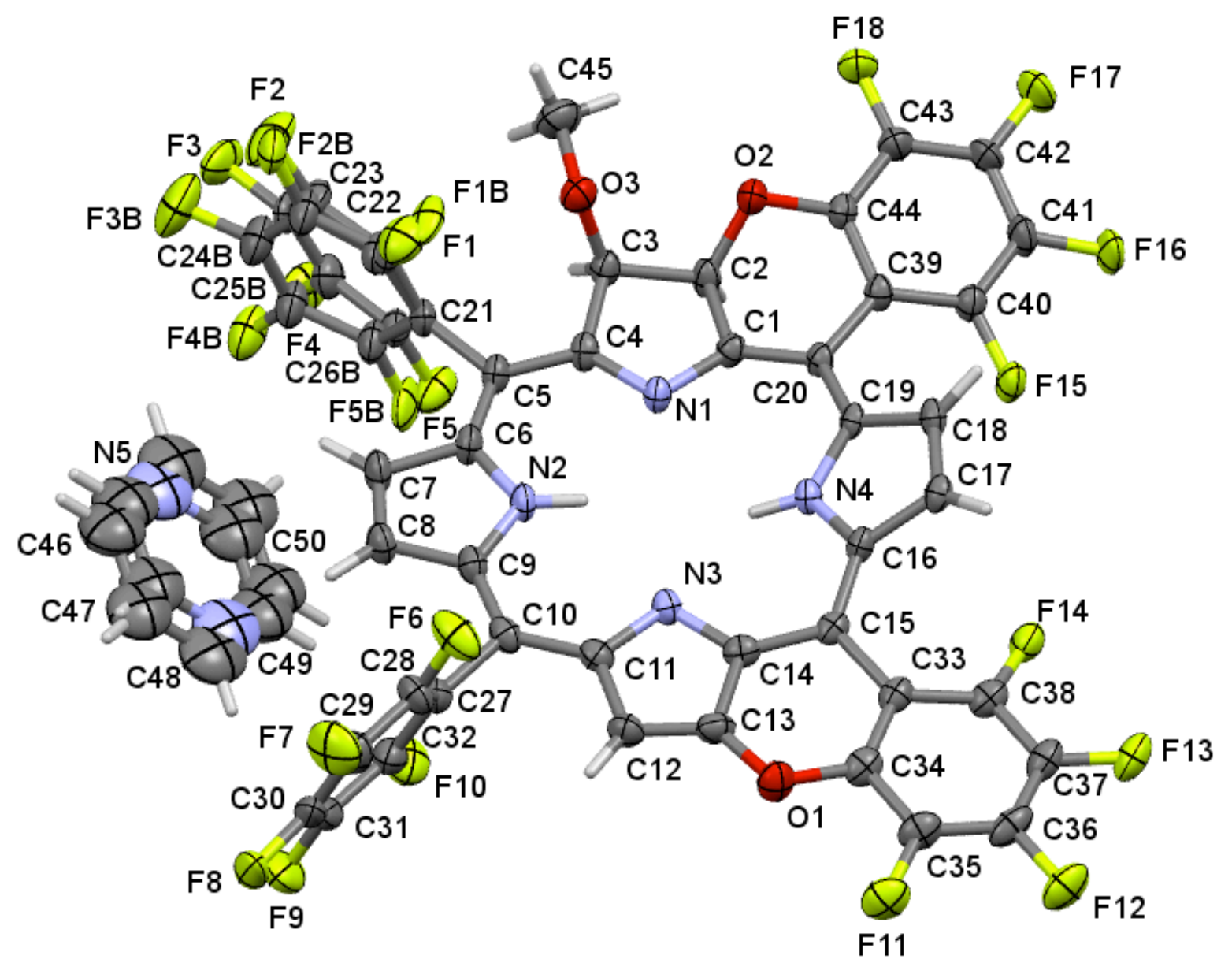

Figure S70. Thermal ellipsoid representation of 14-syn, at the 50\% thermal probability level. For the disordered pentafluoro benzene rings some labels are omitted for clarity. Only one set of labels is given for the pyridine ring disordered across an inversion center. 


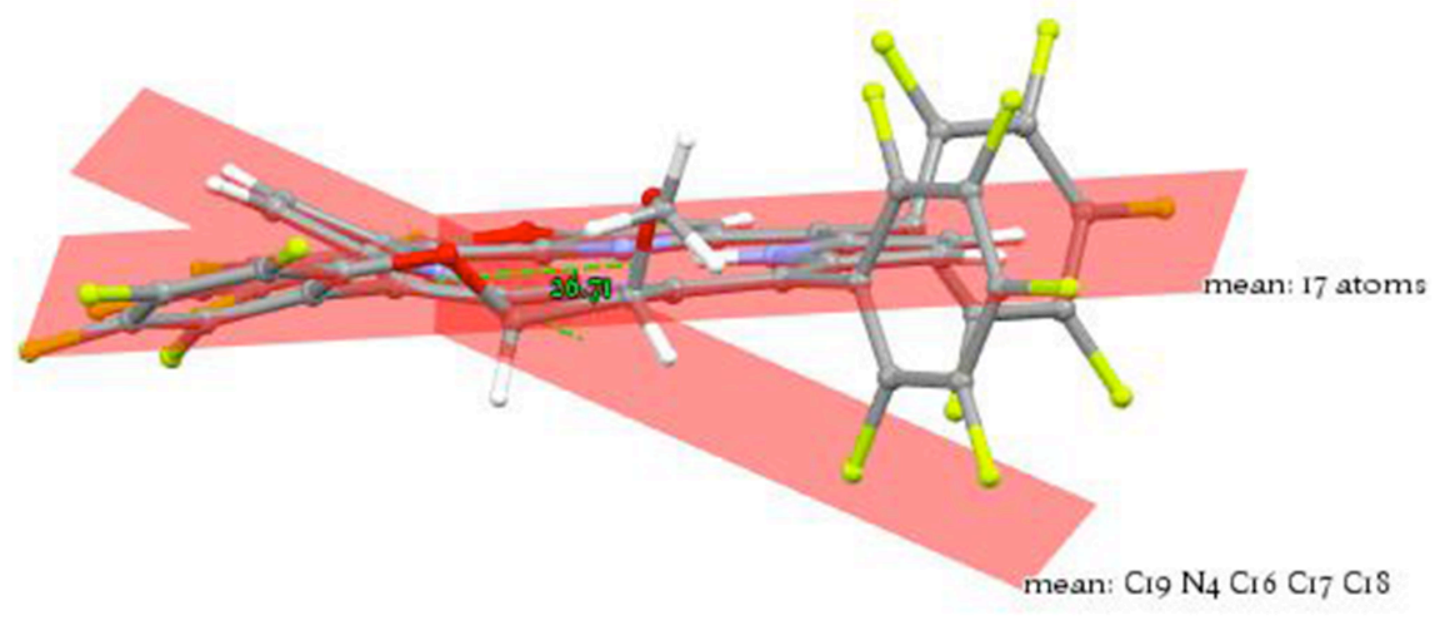

Figure S71. Planes defined to determine the angle at which the mean plane of the pyrrole is angled with respect to the mean plane of the remaining $\mathrm{sp}^{2}$-hybridized heavy atoms of the macrocycle. 


\section{Details to the Computations}

DFT was utilized to determine most energetically favorable geometries (Figure 1) using the GAUSSIAN 09 program package. All calculations were carried out using the B3LYP hybrid functional and the $6-31+\mathrm{G}(\mathrm{d}, \mathrm{p})$ basis set. $^{5}$

Table S1. Energies of Optimized Geometries of Bacteriochlorins Indicated

\begin{tabular}{cc}
\hline $\begin{array}{c}\text { Computed Structure } \\
\text { Number }\end{array}$ & Energy $($ Hartree/kJ/mol) \\
\hline $\mathbf{7}$ & $-3949.713652 / 10369973.9$ \\
$\mathbf{1 2}$ & $-3849.248263 / 10106202.1$ \\
$\mathbf{1 0 - E}$ & $-4101.364827 / 10768134.1736$ \\
$\mathbf{1 0 - Z}$ & $-4101.364391 / 10768133.0288$ \\
$\mathbf{1 1 - E - a d j}$ & $-4000.899465 / 10504362.3455$ \\
$\mathbf{1 1 - Z - a d j}$ & $-4000.899531 / 10504362.5188$ \\
11-E-anti & $-4000.911716 / 10504394.5105$ \\
11-E-syn & $-4000.903546 / 10504373.0602$ \\
11-Z-anti & $-4000.917703 / 10504410.2294$ \\
11-Z-syn & $-4000.905199 / 10504377.4002$
\end{tabular}

${ }^{5}$ Gaussian 09, Revision A.02, Frisch, M. J.; Trucks, G. W.; Schlegel, H. B.; Scuseria, G. E.; Robb, M. A.; Cheeseman, J. R.; Scalmani, G.; Barone, V.; Mennucci, B.; Petersson, G. A.; Nakatsuji, H.; Caricato, M.; Li, X.; Hratchian, H. P.; Izmaylov, A. F.; Bloino, J.; Zheng, G.; Sonnenberg, J. L.; Hada, M.; Ehara, M.; Toyota, K.; Fukuda, R.; Hasegawa, J.; Ishida, M.; Nakajima, T.; Honda, Y.; Kitao, O.; Nakai, H.; Vreven, T.; Montgomery, J. A., Jr.; Peralta, J. E.; Ogliaro, F.; Bearpark, M.; Heyd, J. J.; Brothers, E.; Kudin, K. N.; Staroverov, V. N.; Kobayashi, R.; Normand, J.; Raghavachari, K.; Rendell, A.; Burant, J. C.; Iyengar, S. S.; Tomasi, J.; Cossi, M.; Rega, N.; Millam, J. M.; Klene, M.; Knox, J. E.; Cross, J. B.; Bakken, V.; Adamo, C.; Jaramillo, J.; Gomperts, R.; Stratmann, R. E.; Yazyev, O.; Austin, A. J.; Cammi, R.; Pomelli, C.; Ochterski, J. W.; Martin, R. L.; Morokuma, K.; Zakrzewski, V. G.; Voth, G. A.; Salvador, P.; Dannenberg, J. J.; Dapprich, S.; Daniels, A. D.; Farkas, Ö.; Foresman, J. B.; Ortiz, J. V.; Cioslowski, J.; Fox, D. J. Gaussian, Inc., Wallingford CT, 2009. 


\section{Optimized Geometries/Coordinates of Compounds Indicated}

(Shown as their most stable conformation: dark green $=\mathrm{C}$, red $=\mathrm{O}$, light green $=\mathrm{F}$, blue $=\mathrm{N}$, white $=\mathrm{H})$

\section{Optimized coordinates of compound 7}

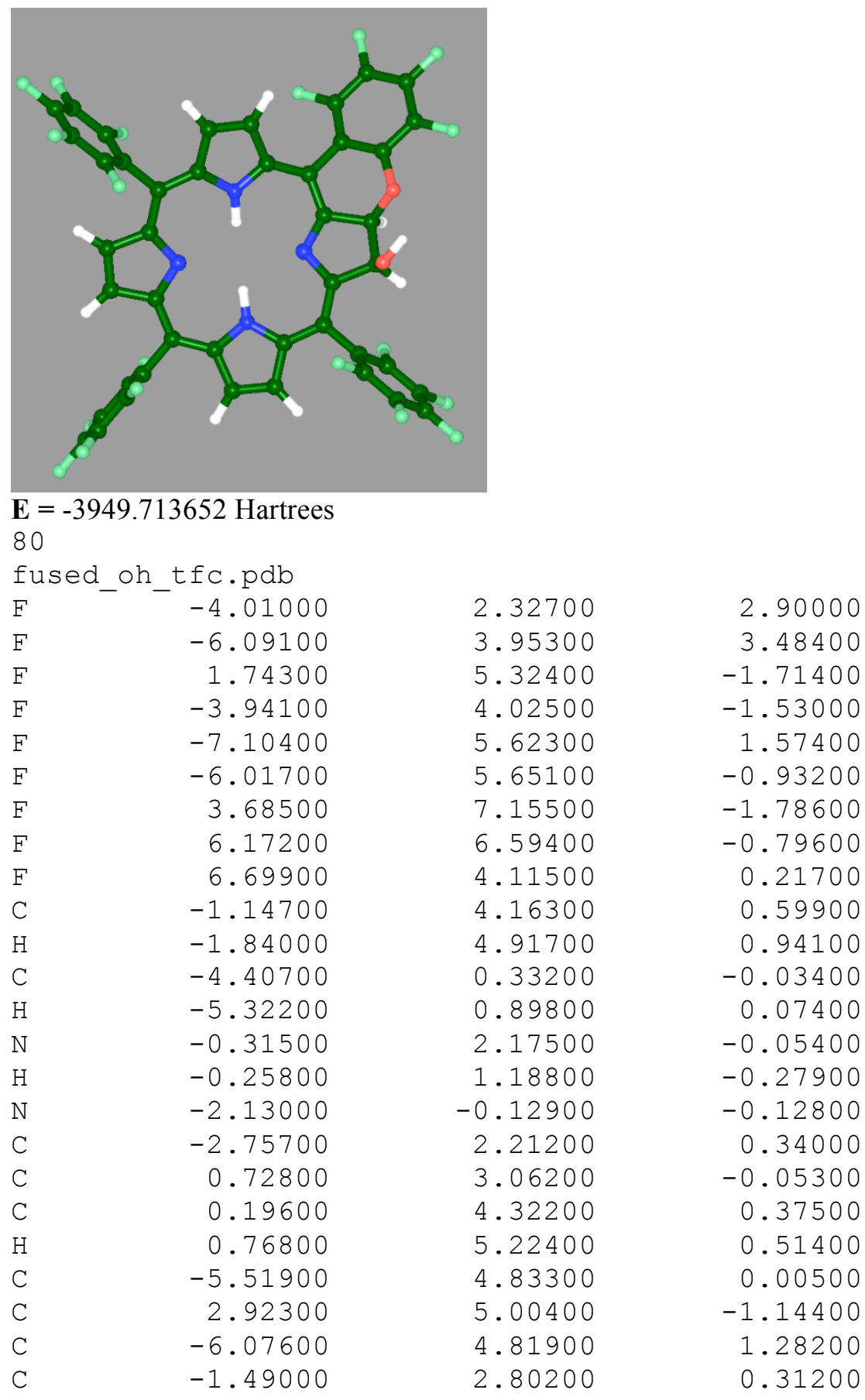




\begin{tabular}{|c|c|c|c|}
\hline C & 4.44700 & 3.45800 & -0.13700 \\
\hline C & 2.07400 & 2.71600 & -0.34600 \\
\hline C & -3.90400 & 3.12000 & 0.66500 \\
\hline C & -3.05300 & 0.85500 & 0.07100 \\
\hline C & -5.55900 & 3.96500 & 2.25400 \\
\hline C & -4.44700 & 3.99100 & -0.28600 \\
\hline C & -4.28200 & -0.99300 & -0.30700 \\
\hline $\mathrm{H}$ & -5.07600 & -1.71300 & -0.45300 \\
\hline C & 3.12000 & 3.75000 & -0.54300 \\
\hline C & -4.48800 & 3.13200 & 1.93700 \\
\hline C & 3.92800 & 5.96500 & -1.21600 \\
\hline C & 2.56200 & 1.40500 & -0.31000 \\
\hline C & 5.45800 & 4.41500 & -0.20100 \\
\hline $\mathrm{C}$ & 5.19800 & 5.67900 & -0.72400 \\
\hline F & 4.46800 & -2.90500 & -1.87600 \\
\hline $\mathrm{F}$ & 6.49600 & -4.62500 & -1.38100 \\
\hline F & -3.45600 & -4.16300 & 1.57800 \\
\hline $\mathrm{F}$ & 3.01900 & -3.34100 & 2.62100 \\
\hline F & 6.80000 & -5.70700 & 1.11100 \\
\hline F & 5.05300 & -5.05500 & 3.10500 \\
\hline $\mathrm{F}$ & -5.18900 & -6.19200 & 1.12600 \\
\hline F & -3.19400 & -3.36000 & -3.08900 \\
\hline $\mathrm{F}$ & -5.93400 & -6.81400 & -1.42800 \\
\hline F & -4.92800 & -5.38900 & -3.53100 \\
\hline $\mathrm{C}$ & 0.97400 & -4.08900 & -0.25100 \\
\hline $\mathrm{H}$ & 1.70600 & -4.88200 & -0.22400 \\
\hline $\mathrm{N}$ & 0.08300 & -2.02500 & -0.20200 \\
\hline $\mathrm{H}$ & -0.03900 & -1.02300 & -0.10500 \\
\hline $\mathrm{N}$ & 1.91000 & 0.24000 & -0.15500 \\
\hline C & 2.54500 & -2.11100 & 0.09800 \\
\hline C & -0.95900 & -2.90800 & -0.40800 \\
\hline $\mathrm{C}$ & -0.37800 & -4.21600 & -0.45500 \\
\hline $\mathrm{H}$ & -0.92800 & -5.13200 & -0.61500 \\
\hline $\mathrm{C}$ & 4.90200 & -4.52400 & 1.88400 \\
\hline C & -3.80200 & -4.44500 & 0.31100 \\
\hline C & 5.79600 & -4.85700 & 0.86900 \\
\hline C & 1.26900 & -2.69600 & -0.09700 \\
\hline C & -3.67100 & -4.04000 & -2.03400 \\
\hline C & -2.31100 & -2.56300 & -0.49900 \\
\hline C & 3.67300 & -3.06200 & 0.35700 \\
\hline C & 2.82300 & -0.74900 & 0.05000 \\
\hline C & 5.63900 & -4.30500 & -0.40100 \\
\hline C & 3.86100 & -3.63700 & 1.62000 \\
\hline C & -3.26800 & -3.69300 & -0.74100 \\
\hline $\mathrm{C}$ & 4.58700 & -3.42400 & -0.63800 \\
\hline C & -4.69600 & -5.49200 & 0.09500 \\
\hline C & -2.85400 & -1.26900 & -0.34200 \\
\hline C & -4.56400 & -5.08200 & -2.27800 \\
\hline C & -5.07700 & -5.81100 & -1.20700 \\
\hline C & 4.23000 & -0.18100 & 0.27000 \\
\hline 0 & 4.45700 & -0.10800 & 1.67100 \\
\hline C & 4.05700 & 1.19600 & -0.38200 \\
\hline
\end{tabular}




$\begin{array}{rrrr}\mathrm{O} & 4.77700 & 2.21200 & 0.32200 \\ \mathrm{H} & 5.07400 & 0.61800 & 1.84600 \\ \mathrm{H} & 5.02000 & -0.76300 & -0.21600 \\ \mathrm{H} & 4.40500 & 1.17300 & -1.42700\end{array}$

\section{Optimized coordinates of compound 12}

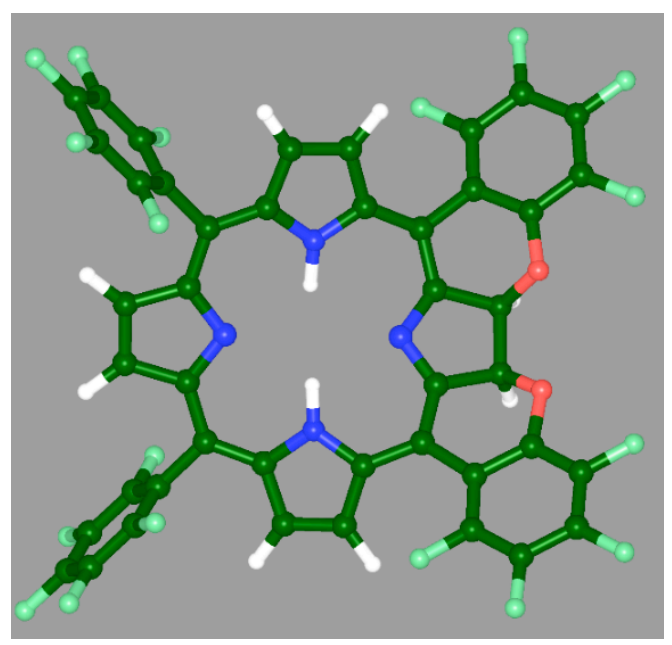

$$
\mathbf{E}=-3849.248263 \text { Hartrees }
$$

78

fused_2_TFC.pdb

$\begin{array}{rrrr}\text { F } & -3.08500 & 3.01300 & 3.00500 \\ F & -4.59200 & 5.11600 & 3.79000 \\ F & 3.12700 & 4.55200 & -1.98300 \\ F & -2.71800 & 4.91000 & -1.33000 \\ F & -5.16400 & 7.12600 & 2.03100 \\ F & -4.21700 & 7.01200 & -0.52900 \\ F & 5.54000 & 5.71400 & -1.96000 \\ F & 7.73300 & 4.35100 & -1.06700 \\ F & 7.49100 & 1.76000 & -0.26300 \\ C & 0.09300 & 4.13700 & 0.51000 \\ H & -0.32200 & 5.04000 & 0.93200 \\ C & -4.13400 & 1.36100 & 0.06500 \\ H & -4.85800 & 2.14700 & 0.22700 \\ \mathrm{~N} & 0.26100 & 2.03300 & -0.26800 \\ \mathrm{H} & 0.01200 & 1.07600 & -0.49800 \\ \mathrm{~N} & -2.07100 & 0.30700 & -0.14000 \\ \mathrm{C} & -2.02100 & 2.71900 & 0.37500 \\ \mathrm{C} & 1.51100 & 2.58500 & -0.34700 \\ \mathrm{C} & 1.40300 & 3.91900 & 0.15600 \\ \mathrm{H} & 2.21700 & 4.61800 & 0.25300 \\ \mathrm{C} & -3.93400 & 6.02700 & 0.33400 \\ \mathrm{C} & 4.16000 & 3.84800 & -1.47600 \\ \mathrm{C} & -4.42100 & 6.08600 & 1.63900 \\ \mathrm{C} & -0.64600 & 2.94500 & 0.23100 \\ \mathrm{C} & 5.14600 & 1.83100 & -0.62600 \\ \mathrm{C} & 2.66500 & 1.87300 & -0.78600 \\ \mathrm{C} & -2.84800 & 3.88800 & 0.81000 \\ \mathrm{C} & -2.68700 & 1.50000 & 0.12000\end{array}$




\begin{tabular}{|c|c|c|c|}
\hline C & -4.12900 & 5.05800 & 2.53400 \\
\hline C & -3.15900 & 4.93800 & -0.06100 \\
\hline C & -4.38000 & 0.06200 & -0.25500 \\
\hline $\mathrm{H}$ & -5.34200 & -0.41200 & -0.38500 \\
\hline $\mathrm{C}$ & 3.97300 & 2.54800 & -0.98300 \\
\hline C & -3.35400 & 3.98000 & 2.11200 \\
\hline $\mathrm{C}$ & 5.40800 & 4.46400 & -1.49100 \\
\hline $\mathrm{C}$ & 2.73300 & 0.48300 & -0.84000 \\
\hline C & 6.39700 & 2.44600 & -0.62600 \\
\hline C & 6.52900 & 3.76800 & -1.04300 \\
\hline$F$ & 6.51100 & -3.68200 & 1.36200 \\
\hline$F$ & -4.08700 & -2.09200 & -3.16300 \\
\hline $\mathrm{F}$ & 1.35300 & -5.38100 & 1.60300 \\
\hline$F$ & 5.87800 & -6.03300 & 2.58800 \\
\hline $\mathrm{F}$ & 3.25900 & -6.83800 & 2.68200 \\
\hline F & -6.30200 & -3.53000 & -3.74600 \\
\hline$F$ & -4.32800 & -3.56700 & 1.34000 \\
\hline $\mathrm{F}$ & -7.53800 & -4.99400 & -1.79900 \\
\hline $\mathrm{F}$ & -6.53800 & -5.00600 & 0.74400 \\
\hline C & -0.18300 & -4.35700 & -0.31800 \\
\hline $\mathrm{H}$ & 0.29600 & -5.32000 & -0.36100 \\
\hline $\mathrm{N}$ & -0.48400 & -2.13900 & -0.12500 \\
\hline $\mathrm{H}$ & -0.33800 & -1.14500 & 0.02100 \\
\hline $\mathrm{N}$ & 1.79400 & -0.42500 & -0.46600 \\
\hline C & 1.86000 & -2.83300 & 0.15100 \\
\hline $\mathrm{C}$ & -1.70800 & -2.68000 & -0.46500 \\
\hline C & -1.49600 & -4.08800 & -0.61200 \\
\hline $\mathrm{H}$ & -2.24900 & -4.80200 & -0.91200 \\
\hline $\mathrm{C}$ & 3.59700 & -5.71000 & 2.03700 \\
\hline C & -4.67300 & -2.79700 & -2.18100 \\
\hline C & 4.92300 & -5.29700 & 2.00900 \\
\hline C & 0.46900 & -3.11700 & -0.00800 \\
\hline C & -4.78900 & -3.54400 & 0.07900 \\
\hline C & -2.90900 & -1.97400 & -0.57100 \\
\hline C & 2.88400 & -3.75600 & 0.70600 \\
\hline C & 2.38900 & -1.61600 & -0.31300 \\
\hline C & 5.23800 & -4.10500 & 1.36800 \\
\hline C & 2.61500 & -4.94400 & 1.41900 \\
\hline C & -4.13100 & -2.78100 & -0.89100 \\
\hline C & 4.25100 & -3.34000 & 0.74500 \\
\hline C & -5.81300 & -3.53300 & -2.49800 \\
\hline C & -3.08000 & -0.58400 & -0.35700 \\
\hline C & -5.93100 & -4.28900 & -0.21200 \\
\hline C & -6.44400 & -4.28300 & -1.50700 \\
\hline C & 3.84200 & -1.61500 & -0.75000 \\
\hline 0 & 4.68900 & -2.14300 & 0.25300 \\
\hline C & 4.09200 & -0.14000 & -1.09800 \\
\hline O & 5.08200 & 0.50700 & -0.29000 \\
\hline $\mathrm{H}$ & 4.39800 & -0.04200 & -2.14800 \\
\hline $\mathrm{H}$ & 3.95600 & -2.23000 & -1.65500 \\
\hline
\end{tabular}




\section{Optimized coordinates of compound 10-E}

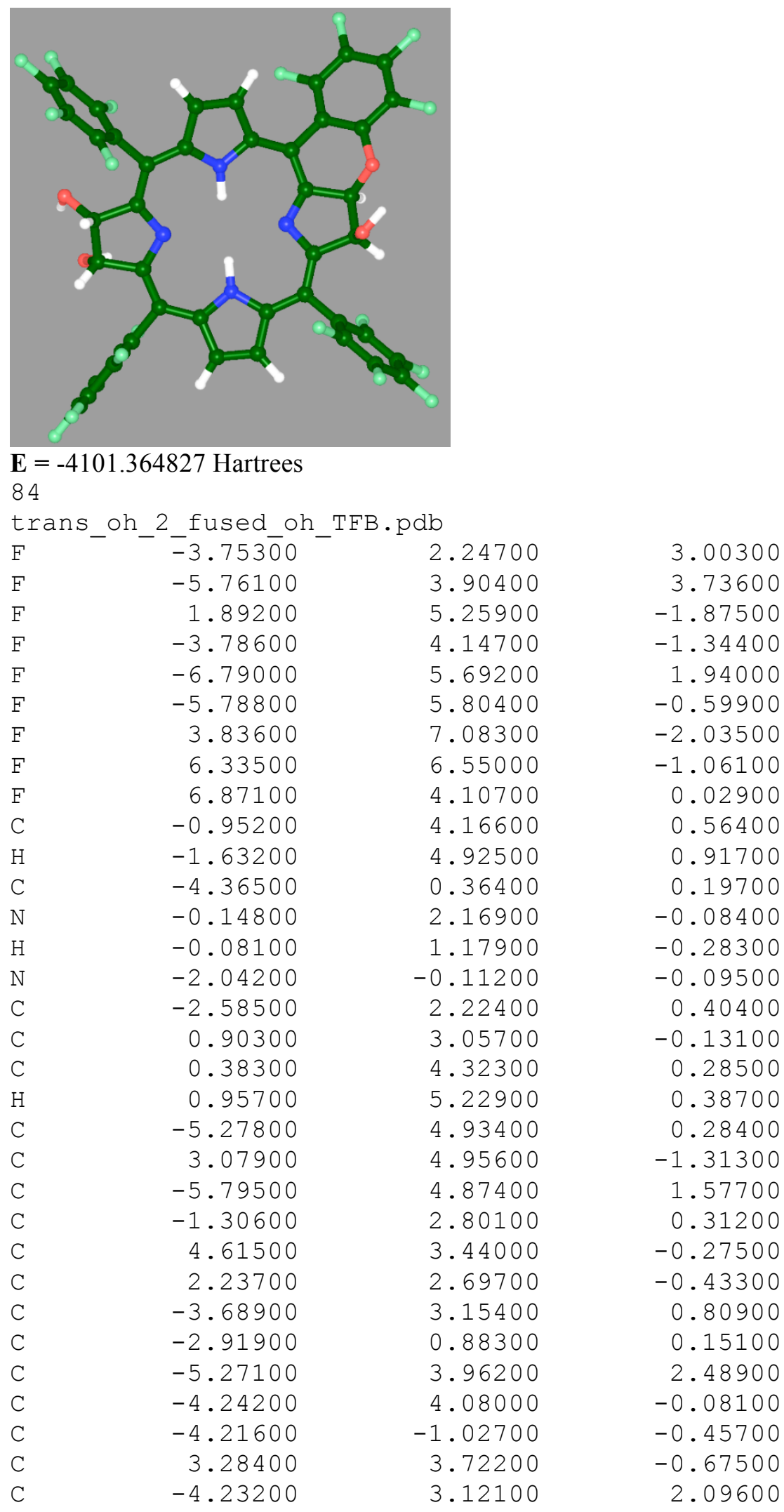




\begin{tabular}{|c|c|c|c|}
\hline C & 4.08500 & 5.91200 & -1.43000 \\
\hline C & 2.72600 & 1.38300 & -0.35700 \\
\hline $\mathrm{C}$ & 5.62600 & 4.39300 & -0.38300 \\
\hline $\mathrm{C}$ & 5.36100 & 5.63900 & -0.94500 \\
\hline $\mathrm{F}$ & 3.17500 & -3.22800 & 2.77300 \\
\hline $\mathrm{F}$ & 5.19900 & -4.93200 & 3.32700 \\
\hline $\mathrm{F}$ & -2.88700 & -3.36100 & -3.08600 \\
\hline $\mathrm{F}$ & 4.61400 & -3.00100 & -1.74300 \\
\hline $\mathrm{F}$ & 6.93700 & -5.68300 & 1.35900 \\
\hline $\mathrm{F}$ & 6.63300 & -4.71100 & -1.17800 \\
\hline $\mathrm{F}$ & -4.55700 & -5.42200 & -3.62200 \\
\hline $\mathrm{F}$ & -3.43200 & -4.13700 & 1.56100 \\
\hline $\mathrm{F}$ & -5.66500 & -6.85400 & -1.57500 \\
\hline $\mathrm{F}$ & -5.09300 & -6.20200 & 1.01500 \\
\hline C & 1.11500 & -4.08600 & -0.07800 \\
\hline $\mathrm{H}$ & 1.83400 & -4.88900 & -0.01100 \\
\hline $\mathrm{N}$ & 0.23900 & -2.01800 & -0.11600 \\
\hline $\mathrm{H}$ & 0.14800 & -1.01200 & -0.06400 \\
\hline $\mathrm{N}$ & 2.07500 & 0.23100 & -0.15300 \\
\hline C & 2.70100 & -2.11000 & 0.19800 \\
\hline C & -0.80300 & -2.89500 & -0.31100 \\
\hline C & -0.24200 & -4.20500 & -0.30000 \\
\hline $\mathrm{H}$ & -0.79800 & -5.12000 & -0.43400 \\
\hline $\mathrm{C}$ & 5.78100 & -4.34200 & -0.21000 \\
\hline $\mathrm{C}$ & -3.41700 & -4.04700 & -2.05700 \\
\hline C & 5.93700 & -4.83900 & 1.08200 \\
\hline $\mathrm{C}$ & 1.42400 & -2.70000 & 0.02800 \\
\hline $\mathrm{C}$ & -3.68600 & -4.43800 & 0.27500 \\
\hline $\mathrm{C}$ & -2.16500 & -2.54400 & -0.45800 \\
\hline C & 3.82500 & -3.05400 & 0.49700 \\
\hline $\mathrm{C}$ & 2.98800 & -0.75500 & 0.09000 \\
\hline $\mathrm{C}$ & 5.04800 & -4.45500 & 2.08400 \\
\hline $\mathrm{C}$ & 4.73400 & -3.46500 & -0.48300 \\
\hline C & -3.09800 & -3.68400 & -0.74500 \\
\hline $\mathrm{C}$ & 4.01100 & -3.57400 & 1.78400 \\
\hline C & -4.27400 & -5.10500 & -2.35100 \\
\hline C & -2.72200 & -1.27100 & -0.33400 \\
\hline $\mathrm{C}$ & -4.54700 & -5.50200 & 0.01200 \\
\hline C & -4.84100 & -5.83600 & -1.30900 \\
\hline $\mathrm{C}$ & 4.39500 & -0.18000 & 0.28100 \\
\hline O & 4.62600 & -0.04600 & 1.67800 \\
\hline $\mathrm{C}$ & 4.22100 & 1.16900 & -0.43000 \\
\hline O & 4.95100 & 2.21200 & 0.22400 \\
\hline $\mathrm{H}$ & 5.26500 & 0.66900 & 1.81800 \\
\hline $\mathrm{H}$ & 5.18300 & -0.78200 & -0.18200 \\
\hline $\mathrm{H}$ & 4.56100 & 1.09900 & -1.47400 \\
\hline $\mathrm{H}$ & -4.65600 & 0.24100 & 1.24900 \\
\hline $\mathrm{H}$ & -4.83500 & -1.78000 & 0.03200 \\
\hline 0 & -5.34000 & 1.16700 & -0.43000 \\
\hline 0 & -4.66000 & -0.98300 & -1.82700 \\
\hline $\mathrm{H}$ & -3.90000 & -0.86700 & -2.41500 \\
\hline $\mathrm{H}$ & -5.45900 & 0.80200 & -1.32400 \\
\hline
\end{tabular}




\section{Optimized coordinates of compound 10-Z}

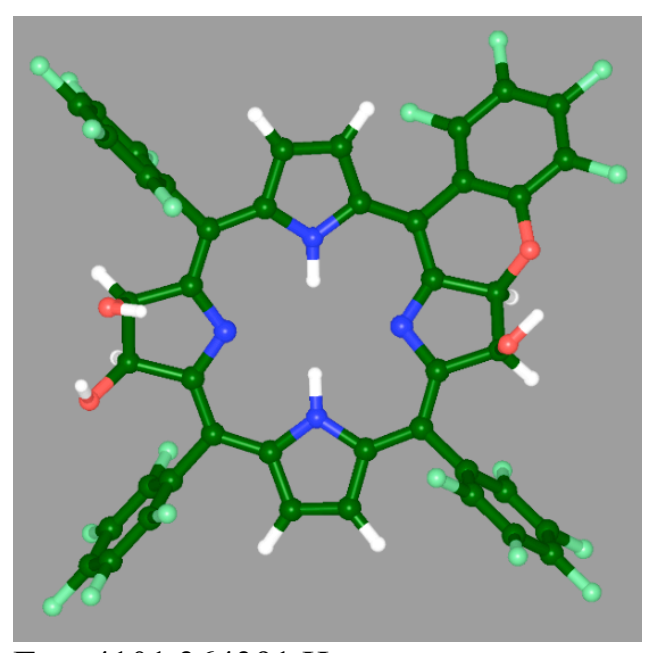

$\begin{array}{lrrr}\mathbf{E}=-4101.364391 \text { Hartrees } & & \\ \text { 84 } & & \\ \text { Cis_oh_2_fused_oh_TFB.pdb } & \\ \text { F } & -3.27200 & 2.91100 & 3.00700 \\ \text { F } & -5.15900 & 4.73400 & 3.66900 \\ \text { F } & 2.27400 & 5.12200 & -1.98000 \\ \text { F } & -3.82600 & 4.01300 & -1.57300 \\ \text { F } & -6.38300 & 6.20900 & 1.72100 \\ \text { F } & -5.70600 & 5.84000 & -0.90000 \\ \text { F } & 4.33900 & 6.80700 & -2.13200 \\ \text { F } & 6.77700 & 6.12700 & -1.09800 \\ \text { F } & 7.12800 & 3.67400 & 0.04300 \\ \text { C } & -0.69800 & 4.25500 & 0.41800 \\ \text { H } & -1.34000 & 5.06600 & 0.72800 \\ \text { C } & -4.31000 & 0.67500 & 0.21500 \\ \text { N } & 0.00300 & 2.19700 & -0.14000 \\ \text { H } & 0.02000 & 1.20000 & -0.31300 \\ \text { N } & -2.04600 & 0.00900 & -0.12900 \\ \text { C } & -2.43000 & 2.39700 & 0.33300 \\ \text { C } & 1.10300 & 3.02400 & -0.20700 \\ \text { C } & 0.65100 & 4.32800 & 0.15400 \\ \text { H } & 1.27100 & 5.20600 & 0.22800 \\ \text { C } & -5.10400 & 5.11900 & 0.05500 \\ \text { C } & 3.42700 & 4.75000 & -1.38800 \\ \text { C } & -5.45100 & 5.30800 & 1.39200 \\ \text { C } & -1.11900 & 2.90600 & 0.22000 \\ \text { C } & 4.83800 & 3.15400 & -0.29500 \\ \text { C } & 2.41900 & 2.57100 & -0.47900 \\ \text { C } & -3.48300 & 3.40500 & 0.69500 \\ \text { C } & -2.84700 & 1.07800 & 0.12500 \\ \text { C } & -4.82600 & 4.55500 & 2.38400 \\ \text { C } & -4.13200 & 4.17700 & -0.27300 \\ \text { C } & -4.29600 & -0.71500 & -0.45600 \\ \text { C } & 3.53600 & 3.51700 & -0.72400 \\ \text { C } & -3.85800 & 3.62100 & 2.02400 \\ & & & \end{array}$




\begin{tabular}{|c|c|c|c|}
\hline C & 4.49700 & 5.63400 & -1.50200 \\
\hline C & 2.81700 & 1.23300 & -0.37300 \\
\hline $\mathrm{C}$ & 5.91200 & 4.03600 & -0.39800 \\
\hline $\mathrm{C}$ & 5.74200 & 5.28600 & -0.98600 \\
\hline $\mathrm{F}$ & 4.43600 & -3.24500 & -1.71500 \\
\hline $\mathrm{F}$ & 6.33200 & -5.08300 & -1.12900 \\
\hline $\mathrm{F}$ & -3.37500 & -2.78800 & -3.13300 \\
\hline $\mathrm{F}$ & 2.89900 & -3.41700 & 2.77000 \\
\hline $\mathrm{F}$ & 6.52600 & -6.09300 & 1.40400 \\
\hline $\mathrm{F}$ & 4.80100 & -5.24900 & 3.34600 \\
\hline $\mathrm{F}$ & -5.16500 & -4.71500 & -3.76600 \\
\hline $\mathrm{F}$ & -3.40200 & -4.25600 & 1.37700 \\
\hline $\mathrm{F}$ & -6.07900 & -6.42500 & -1.83700 \\
\hline $\mathrm{F}$ & -5.18400 & -6.18600 & 0.73400 \\
\hline C & 0.85500 & -4.12200 & -0.15300 \\
\hline $\mathrm{H}$ & 1.52900 & -4.96400 & -0.10100 \\
\hline $\mathrm{N}$ & 0.09700 & -2.00600 & -0.13800 \\
\hline $\mathrm{H}$ & 0.05700 & -0.99700 & -0.07200 \\
\hline $\mathrm{N}$ & 2.08400 & 0.12600 & -0.16300 \\
\hline C & 2.54500 & -2.25100 & 0.19900 \\
\hline C & -0.99000 & -2.81600 & -0.37700 \\
\hline C & -0.49600 & -4.15800 & -0.40500 \\
\hline $\mathrm{H}$ & -1.09900 & -5.03600 & -0.58300 \\
\hline $\mathrm{C}$ & 4.70500 & -4.75400 & 2.10500 \\
\hline $\mathrm{C}$ & -3.79900 & -3.62000 & -2.16100 \\
\hline C & 5.58700 & -5.18500 & 1.11600 \\
\hline C & 1.24100 & -2.75500 & 0.00000 \\
\hline $\mathrm{C}$ & -3.80600 & -4.36100 & 0.09800 \\
\hline $\mathrm{C}$ & -2.32800 & -2.39300 & -0.52000 \\
\hline C & 3.60200 & -3.26700 & 0.50900 \\
\hline $\mathrm{C}$ & 2.92200 & -0.91200 & 0.09900 \\
\hline C & 5.48600 & -4.66900 & -0.17400 \\
\hline $\mathrm{C}$ & 3.73100 & -3.80700 & 1.79300 \\
\hline C & -3.31400 & -3.47000 & -0.86000 \\
\hline $\mathrm{C}$ & 4.50200 & -3.72600 & -0.45800 \\
\hline C & -4.72600 & -4.60100 & -2.50400 \\
\hline C & -2.80800 & -1.08700 & -0.36000 \\
\hline $\mathrm{C}$ & -4.72900 & -5.35300 & -0.21400 \\
\hline C & -5.19200 & -5.47300 & -1.52300 \\
\hline $\mathrm{C}$ & 4.36300 & -0.43300 & 0.30800 \\
\hline O & 4.57700 & -0.29700 & 1.70800 \\
\hline $\mathrm{C}$ & 4.29300 & 0.91500 & -0.42100 \\
\hline O & 5.08200 & 1.91500 & 0.23000 \\
\hline $\mathrm{H}$ & 5.25700 & 0.37700 & 1.85200 \\
\hline $\mathrm{H}$ & 5.11600 & -1.09300 & -0.13200 \\
\hline $\mathrm{H}$ & 4.64300 & 0.80700 & -1.45900 \\
\hline O & -4.77600 & 0.56700 & 1.57300 \\
\hline $\mathrm{H}$ & -4.02300 & 0.47500 & 2.17400 \\
\hline 0 & -5.21900 & -1.60900 & 0.12400 \\
\hline $\mathrm{H}$ & -5.40700 & -1.26700 & 1.01500 \\
\hline $\mathrm{H}$ & -4.99400 & 1.36600 & -0.27900 \\
\hline $\mathrm{H}$ & -4.55500 & -0.61000 & -1.51800 \\
\hline
\end{tabular}




\section{Optimized coordinates of compound $11^{\mathrm{H}}$-E-adj}

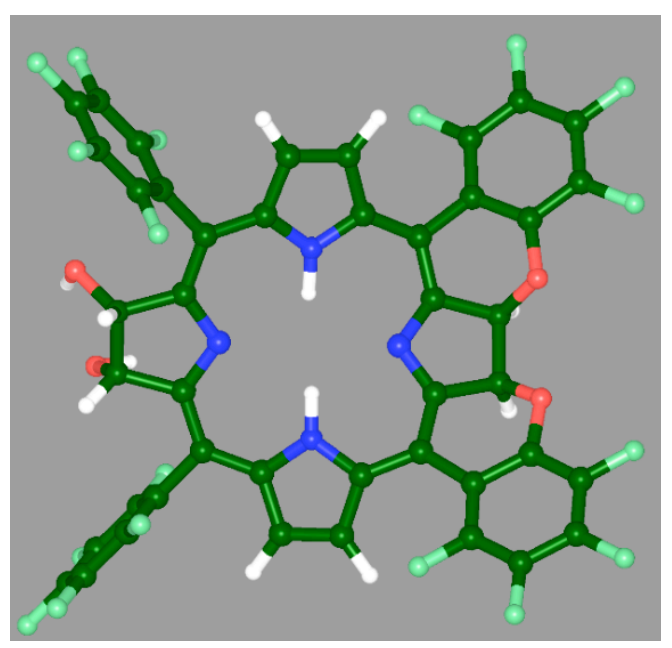

$\mathrm{E}=-4000.899465$ Hartrees

82

trans_oh_2_fused_2_TFC.pdb

\begin{tabular}{|c|c|c|c|}
\hline F & -3.05100 & 2.92600 & 3.02800 \\
\hline F & -4.48900 & 5.03400 & 3.92200 \\
\hline $\mathrm{F}$ & 3.10100 & 4.50600 & -2.10200 \\
\hline F & -2.71700 & 4.97500 & -1.23700 \\
\hline $\mathrm{F}$ & -5.04300 & 7.12500 & 2.24900 \\
\hline F & -4.14500 & 7.08300 & -0.33100 \\
\hline $\mathrm{F}$ & 5.51200 & 5.67100 & -2.11400 \\
\hline $\mathrm{F}$ & 7.71000 & 4.32700 & -1.20600 \\
\hline $\mathrm{F}$ & 7.47500 & 1.75200 & -0.35100 \\
\hline $\mathrm{C}$ & 0.09300 & 4.11700 & 0.45500 \\
\hline $\mathrm{H}$ & -0.31500 & 5.02100 & 0.88300 \\
\hline $\mathrm{C}$ & -4.24900 & 1.38800 & 0.22100 \\
\hline $\mathrm{N}$ & 0.24400 & 2.01700 & -0.32300 \\
\hline $\mathrm{H}$ & 0.01100 & 1.05500 & -0.53500 \\
\hline $\mathrm{N}$ & -2.14600 & 0.32700 & -0.16500 \\
\hline $\mathrm{C}$ & -2.03200 & 2.71100 & 0.37700 \\
\hline C & 1.50200 & 2.57000 & -0.42300 \\
\hline $\mathrm{C}$ & 1.39800 & 3.90300 & 0.07300 \\
\hline $\mathrm{H}$ & 2.21100 & 4.60600 & 0.15300 \\
\hline $\mathrm{C}$ & -3.86500 & 6.06200 & 0.49200 \\
\hline $\mathrm{C}$ & 4.13600 & 3.81200 & -1.58800 \\
\hline $\mathrm{C}$ & -4.33000 & 6.08300 & 1.80600 \\
\hline $\mathrm{C}$ & -0.65000 & 2.92600 & 0.19300 \\
\hline C & 5.12900 & 1.81200 & -0.70600 \\
\hline C & 2.64600 & 1.85000 & -0.85600 \\
\hline C & -2.81800 & 3.88700 & 0.87000 \\
\hline C & -2.72100 & 1.51700 & 0.12900 \\
\hline $\mathrm{C}$ & -4.04800 & 5.01700 & 2.65600 \\
\hline C & -3.12300 & 4.97400 & 0.04400 \\
\hline C & -4.48700 & 0.04500 & -0.50500 \\
\hline C & 3.95400 & 2.52100 & -1.07100 \\
\hline
\end{tabular}




\begin{tabular}{|c|c|c|c|}
\hline $\mathrm{C}$ & -3.30100 & 3.94200 & 2.18000 \\
\hline C & 5.38300 & 4.42900 & -1.62100 \\
\hline C & 2.71700 & 0.45600 & -0.88700 \\
\hline $\mathrm{C}$ & 6.37900 & 2.42900 & -0.72200 \\
\hline $\mathrm{C}$ & 6.50700 & 3.74300 & -1.16500 \\
\hline F & 6.51600 & -3.63500 & 1.41000 \\
\hline F & -3.81100 & -2.34100 & -3.27400 \\
\hline $\mathrm{F}$ & 1.36400 & -5.33500 & 1.74400 \\
\hline $\mathrm{F}$ & 5.89500 & -5.94200 & 2.72200 \\
\hline F & 3.27800 & -6.74800 & 2.86100 \\
\hline $\mathrm{F}$ & -5.96100 & -3.83800 & -3.94900 \\
\hline F & -4.56000 & -3.34600 & 1.30100 \\
\hline F & -7.41500 & -5.10200 & -2.01100 \\
\hline F & -6.70300 & -4.85000 & 0.61400 \\
\hline $\mathrm{C}$ & -0.20100 & -4.36200 & -0.18400 \\
\hline $\mathrm{H}$ & 0.26300 & -5.33400 & -0.17800 \\
\hline $\mathrm{N}$ & -0.48600 & -2.13700 & -0.09800 \\
\hline $\mathrm{H}$ & -0.31100 & -1.14500 & 0.00200 \\
\hline $\mathrm{N}$ & 1.78600 & -0.44400 & -0.48200 \\
\hline C & 1.85400 & -2.83300 & 0.20300 \\
\hline $\mathrm{C}$ & -1.70600 & -2.67900 & -0.43000 \\
\hline $\mathrm{C}$ & -1.51100 & -4.08900 & -0.50900 \\
\hline $\mathrm{H}$ & -2.26900 & -4.80800 & -0.78000 \\
\hline C & 3.61000 & -5.64200 & 2.17500 \\
\hline $\mathrm{C}$ & -4.50800 & -2.95000 & -2.29500 \\
\hline C & 4.93600 & -5.22800 & 2.12400 \\
\hline C & 0.46300 & -3.12400 & 0.07200 \\
\hline $\mathrm{C}$ & -4.87900 & -3.45800 & -0.00000 \\
\hline C & -2.91400 & -1.96800 & -0.59900 \\
\hline C & 2.88500 & -3.73600 & 0.78300 \\
\hline $\mathrm{C}$ & 2.38100 & -1.63100 & -0.30100 \\
\hline C & 5.24400 & -4.05900 & 1.44100 \\
\hline $\mathrm{C}$ & 2.62300 & -4.90000 & 1.53700 \\
\hline C & -4.11000 & -2.79800 & -0.96400 \\
\hline C & 4.25100 & -3.31600 & 0.79700 \\
\hline C & -5.61100 & -3.71700 & -2.66200 \\
\hline C & -3.11100 & -0.59400 & -0.42500 \\
\hline C & -5.98800 & -4.23300 & -0.33700 \\
\hline C & -6.35400 & -4.36200 & -1.67500 \\
\hline C & 3.83100 & -1.63900 & -0.74800 \\
\hline O & 4.68800 & -2.14100 & 0.26000 \\
\hline C & 4.07700 & -0.17100 & -1.13400 \\
\hline $\mathrm{O}$ & 5.06900 & 0.49500 & -0.34400 \\
\hline $\mathrm{H}$ & 4.38100 & -0.09900 & -2.18600 \\
\hline $\mathrm{H}$ & 3.94200 & -2.27500 & -1.63900 \\
\hline $\mathrm{H}$ & -4.52600 & 1.28200 & 1.27800 \\
\hline $\mathrm{H}$ & -5.28000 & -0.54100 & -0.03900 \\
\hline 0 & -5.00600 & 2.44800 & -0.31800 \\
\hline O & -4.91700 & 0.28000 & -1.85900 \\
\hline $\mathrm{H}$ & -5.23000 & 2.18900 & -1.22900 \\
\hline $\mathrm{H}$ & -4.15600 & 0.24300 & -2.45700 \\
\hline
\end{tabular}




\section{Optimized coordinates of compound $11^{\mathrm{H}}-\mathrm{Z}$-adj}

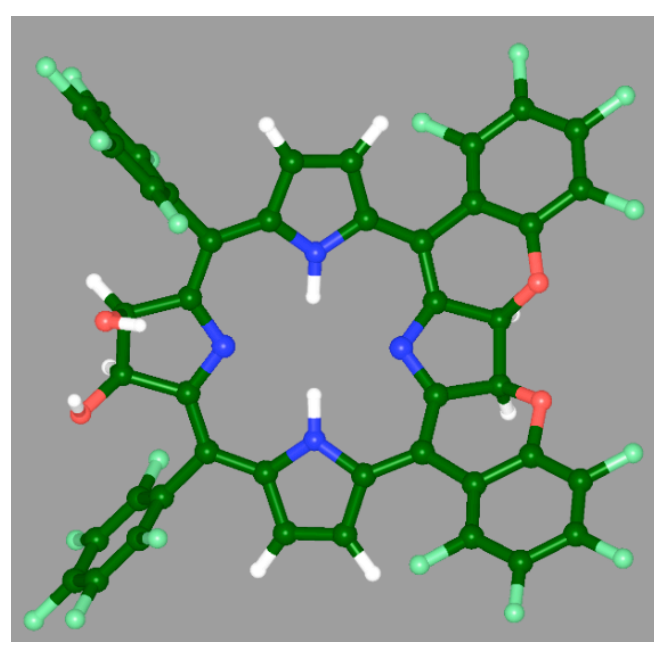

$\begin{array}{lrlr}\text { E = -4000.899531 Hartrees } & & \\ \text { 82 } & & \\ \text { Cis_oh_2_fused_2 TFC.pdb } & & \\ \text { F } & -2.67000 & 3.21500 & 3.14500 \\ \text { F } & -4.08400 & 5.36200 & 3.98900 \\ \text { F } & 3.12400 & 4.51000 & -2.11200 \\ \text { F } & -3.05500 & 4.73500 & -1.33300 \\ \text { F } & -4.98300 & 7.20700 & 2.18500 \\ \text { F } & -4.46000 & 6.88300 & -0.47700 \\ \text { F } & 5.54000 & 5.66200 & -2.13200 \\ \text { F } & 7.73300 & 4.31300 & -1.21800 \\ \text { F } & 7.48700 & 1.74500 & -0.34600 \\ \text { C } & 0.09100 & 4.14900 & 0.43600 \\ \text { H } & -0.32300 & 5.06200 & 0.83800 \\ \text { C } & -4.21700 & 1.41900 & 0.33300 \\ \text { N } & 0.26000 & 2.03300 & -0.28600 \\ \text { H } & 0.03500 & 1.06600 & -0.48300 \\ \text { N } & -2.14700 & 0.31500 & -0.10300 \\ \text { C } & -2.02000 & 2.71700 & 0.41500 \\ \text { C } & 1.51200 & 2.59500 & -0.40700 \\ \text { C } & 1.40100 & 3.93400 & 0.05500 \\ \text { H } & 2.20600 & 4.64800 & 0.11100 \\ \text { C } & -4.01700 & 5.98000 & 0.40800 \\ \text { C } & 4.15600 & 3.81400 & -1.59300 \\ \text { C } & -4.28600 & 6.14700 & 1.76500 \\ \text { C } & -0.63700 & 2.94600 & 0.21300 \\ \text { C } & 5.14000 & 1.81400 & -0.70000 \\ \text { C } & 2.65800 & 1.86400 & -0.83700 \\ \text { C } & -2.81200 & 3.90500 & 0.87800 \\ \text { C } & -2.70600 & 1.52300 & 0.20500 \\ \text { C } & -3.82600 & 5.20500 & 2.68400 \\ \text { C } & -3.28800 & 4.87000 & -0.01600 \\ \text { C } & -4.50100 & 0.08800 & -0.39800 \\ \text { C } & 3.96700 & 2.52700 & -1.06700 \\ \text { C } & -3.10200 & 4.10600 & 2.23000\end{array}$




\begin{tabular}{|c|c|c|c|}
\hline C & 5.40600 & 4.42400 & -1.63100 \\
\hline $\mathrm{C}$ & 2.72200 & 0.47500 & -0.85600 \\
\hline $\mathrm{C}$ & 6.39300 & 2.42500 & -0.72100 \\
\hline C & 6.52700 & 3.73600 & -1.17200 \\
\hline $\mathrm{F}$ & 6.50900 & -3.65800 & 1.40500 \\
\hline F & -4.02200 & -1.94800 & -3.18400 \\
\hline F & 1.34200 & -5.30500 & 1.78100 \\
\hline $\mathrm{F}$ & 5.87600 & -5.95900 & 2.72000 \\
\hline $\mathrm{F}$ & 3.25400 & -6.74000 & 2.88300 \\
\hline $\mathrm{F}$ & -6.17000 & -3.41500 & -3.92700 \\
\hline F & -4.36300 & -3.69300 & 1.21300 \\
\hline F & -7.42100 & -5.03200 & -2.11100 \\
\hline F & -6.50200 & -5.16400 & 0.45900 \\
\hline C & -0.20000 & -4.34300 & -0.17700 \\
\hline $\mathrm{H}$ & 0.27100 & -5.31200 & -0.18000 \\
\hline $\mathrm{N}$ & -0.49600 & -2.11800 & -0.05800 \\
\hline $\mathrm{H}$ & -0.33000 & -1.12500 & 0.05200 \\
\hline $\mathrm{N}$ & 1.78700 & -0.42000 & -0.43000 \\
\hline C & 1.84300 & -2.81300 & 0.24300 \\
\hline C & -1.71200 & -2.66000 & -0.40700 \\
\hline C & -1.50300 & -4.07300 & -0.51300 \\
\hline $\mathrm{H}$ & -2.25300 & -4.79000 & -0.80900 \\
\hline C & 3.59000 & -5.63800 & 2.19500 \\
\hline C & -4.61100 & -2.74000 & -2.26700 \\
\hline C & 4.91900 & -5.23600 & 2.13100 \\
\hline C & 0.45800 & -3.10100 & 0.10200 \\
\hline C & -4.77400 & -3.62300 & -0.06500 \\
\hline C & -2.92300 & -1.96100 & -0.56300 \\
\hline $\mathrm{C}$ & 2.87200 & -3.72300 & 0.81100 \\
\hline C & 2.37500 & -1.60500 & -0.25600 \\
\hline C & 5.23300 & -4.06900 & 1.44600 \\
\hline C & 2.60500 & -4.88500 & 1.56600 \\
\hline C & -4.10700 & -2.78700 & -0.96500 \\
\hline C & 4.24200 & -3.31600 & 0.81300 \\
\hline C & -5.72000 & -3.48300 & -2.66600 \\
\hline C & -3.11900 & -0.58500 & -0.35300 \\
\hline C & -5.88200 & -4.37900 & -0.43400 \\
\hline C & -6.35800 & -4.30800 & -1.74300 \\
\hline C & 3.81800 & -1.62800 & -0.72300 \\
\hline 0 & 4.68400 & -2.14300 & 0.27200 \\
\hline C & 4.07400 & -0.16400 & -1.11300 \\
\hline 0 & 5.07600 & 0.49900 & -0.33200 \\
\hline $\mathrm{H}$ & 4.37200 & -0.09700 & -2.16700 \\
\hline $\mathrm{H}$ & 3.90800 & -2.26500 & -1.61600 \\
\hline $\mathrm{H}$ & -4.75900 & 2.25700 & -0.10800 \\
\hline $\mathrm{H}$ & -4.74900 & 0.29500 & -1.44900 \\
\hline 0 & -4.66500 & 1.33400 & 1.69800 \\
\hline O & -5.57700 & -0.62500 & 0.16400 \\
\hline $\mathrm{H}$ & -3.92200 & 1.10400 & 2.27600 \\
\hline $\mathrm{H}$ & -5.66400 & -0.31000 & 1.08100 \\
\hline
\end{tabular}


Optimized coordinates of compound $11^{\mathrm{H}}-E$-anti

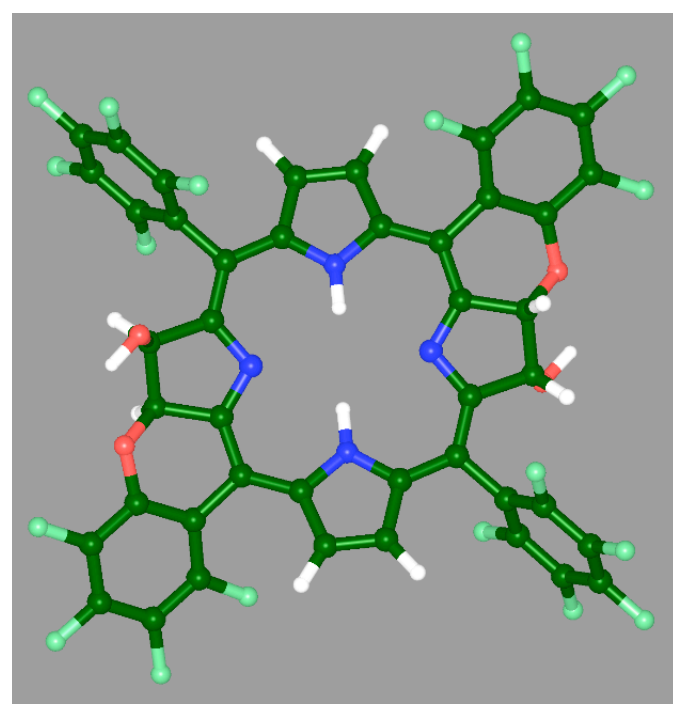

\begin{tabular}{|c|c|c|c|}
\hline \multicolumn{4}{|c|}{$\begin{array}{l}E=-4000.911716 \text { Hartrees } \\
82 \\
\text { trans anti fused oh }\end{array}$} \\
\hline F & $4.628 \overline{0} 0$ & -2.18300 & 2.38500 \\
\hline $\mathrm{F}$ & 6.80500 & -3.78700 & 2.37900 \\
\hline F & -1.64600 & -5.57100 & -1.09000 \\
\hline $\mathrm{F}$ & 3.56200 & -3.63600 & -2.00500 \\
\hline $\mathrm{F}$ & 7.37600 & -5.31700 & 0.18700 \\
\hline F & 5.74300 & -5.23000 & -2.00100 \\
\hline F & -3.46100 & -7.49800 & -0.80100 \\
\hline $\mathrm{F}$ & -5.90700 & -6.96400 & 0.29900 \\
\hline $\mathrm{F}$ & -6.52900 & -4.41100 & 1.03800 \\
\hline $\mathrm{C}$ & 1.36800 & -4.00300 & 0.70500 \\
\hline $\mathrm{H}$ & 2.15300 & -4.69100 & 0.98100 \\
\hline $\mathrm{C}$ & 4.36400 & 0.01500 & -0.28200 \\
\hline $\mathrm{N}$ & 0.32000 & -2.10900 & 0.10900 \\
\hline $\mathrm{H}$ & 0.13900 & -1.14900 & -0.15700 \\
\hline $\mathrm{N}$ & 2.01600 & 0.34600 & 0.06800 \\
\hline $\mathrm{C}$ & 2.80500 & -1.97300 & 0.18400 \\
\hline $\mathrm{C}$ & -0.65400 & -3.07600 & 0.25600 \\
\hline $\mathrm{C}$ & 0.01700 & -4.26400 & 0.67000 \\
\hline $\mathrm{H}$ & -0.45900 & -5.19800 & 0.92000 \\
\hline $\mathrm{C}$ & 5.46200 & -4.48100 & -0.92700 \\
\hline $\mathrm{C}$ & -2.79400 & -5.24900 & -0.46300 \\
\hline $\mathrm{C}$ & 6.29700 & -4.52600 & 0.18700 \\
\hline $\mathrm{C}$ & 1.56800 & -2.64000 & 0.33500 \\
\hline C & -4.34800 & -3.68000 & 0.45800 \\
\hline $\mathrm{C}$ & -2.03500 & -2.84000 & 0.03900 \\
\hline $\mathrm{C}$ & 4.01800 & -2.85300 & 0.18900 \\
\hline $\mathrm{C}$ & 2.99300 & -0.60100 & 0.02300 \\
\hline C & 6.00500 & -3.74500 & 1.30400 \\
\hline $\mathrm{C}$ & 4.34100 & -3.65300 & -0.91400 \\
\hline $\mathrm{C}$ & 4.09600 & 1.46400 & 0.13200 \\
\hline C & -3.02700 & -3.94600 & 0.00900 \\
\hline
\end{tabular}




\begin{tabular}{|c|c|c|c|}
\hline C & 4.87800 & -2.92700 & 1.29000 \\
\hline $\mathrm{C}$ & -3.74000 & -6.26500 & -0.35100 \\
\hline C & -2.60700 & -1.56300 & -0.05000 \\
\hline $\mathrm{C}$ & -5.29700 & -4.69200 & 0.58200 \\
\hline C & -4.99100 & -5.99400 & 0.19600 \\
\hline F & -3.57800 & 3.63500 & 2.01500 \\
\hline F & -5.75900 & 5.22900 & 2.01100 \\
\hline F & -4.64400 & 2.18000 & -2.37400 \\
\hline F & -7.39100 & 5.31400 & -0.17700 \\
\hline F & -6.82100 & 3.78300 & -2.36900 \\
\hline F & 6.51300 & 4.40900 & -1.02600 \\
\hline F & 1.63000 & 5.56900 & 1.10100 \\
\hline F & 5.89100 & 6.96200 & -0.28700 \\
\hline F & 3.44500 & 7.49600 & 0.81200 \\
\hline $\mathrm{C}$ & -1.38300 & 4.00100 & -0.69500 \\
\hline $\mathrm{H}$ & -2.16800 & 4.68900 & -0.97100 \\
\hline $\mathrm{N}$ & -0.33500 & 2.10700 & -0.09800 \\
\hline $\mathrm{H}$ & -0.15400 & 1.14700 & 0.16800 \\
\hline $\mathrm{N}$ & -2.03200 & -0.34800 & -0.05800 \\
\hline C & -2.82000 & 1.97000 & -0.17300 \\
\hline C & 0.63800 & 3.07300 & -0.24600 \\
\hline C & -0.03300 & 4.26200 & -0.65900 \\
\hline $\mathrm{H}$ & 0.44400 & 5.19500 & -0.91000 \\
\hline C & -6.02000 & 3.74200 & -1.29400 \\
\hline $\mathrm{C}$ & 4.33200 & 3.67800 & -0.44700 \\
\hline C & -6.31300 & 4.52300 & -0.17800 \\
\hline C & -1.58300 & 2.63800 & -0.32400 \\
\hline C & 2.77800 & 5.24700 & 0.47400 \\
\hline C & 2.01900 & 2.83700 & -0.02800 \\
\hline $\mathrm{C}$ & -4.03300 & 2.85100 & -0.17900 \\
\hline C & -3.00800 & 0.59900 & -0.01200 \\
\hline C & -5.47700 & 4.47900 & 0.93700 \\
\hline C & -4.89300 & 2.92400 & -1.27900 \\
\hline $\mathrm{C}$ & 3.01200 & 3.94400 & 0.00200 \\
\hline C & -4.35700 & 3.65100 & 0.92400 \\
\hline C & 5.28200 & 4.69000 & -0.57000 \\
\hline C & 2.59100 & 1.56000 & 0.06100 \\
\hline C & 3.72400 & 6.26300 & 0.36200 \\
\hline $\mathrm{C}$ & 4.97500 & 5.99200 & -0.18500 \\
\hline C & -4.37900 & -0.01700 & 0.29200 \\
\hline 0 & -4.61600 & 0.13400 & 1.68700 \\
\hline C & -4.11200 & -1.46700 & -0.12100 \\
\hline 0 & -4.73900 & -2.40500 & 0.76000 \\
\hline $\mathrm{H}$ & -5.18400 & -0.59300 & 1.98500 \\
\hline $\mathrm{H}$ & -5.20000 & 0.42200 & -0.28200 \\
\hline $\mathrm{H}$ & -4.47500 & -1.64800 & -1.14400 \\
\hline $\mathrm{H}$ & 5.18400 & -0.42400 & 0.29300 \\
\hline O & 4.60000 & -0.13600 & -1.67600 \\
\hline 0 & 4.72300 & 2.40300 & -0.74900 \\
\hline $\mathrm{H}$ & 5.16900 & 0.59000 & -1.97300 \\
\hline $\mathrm{H}$ & 4.45900 & 1.64500 & 1.15500 \\
\hline
\end{tabular}




\section{Optimized coordinates of compound $11^{\mathrm{H}}-E-s y n$}

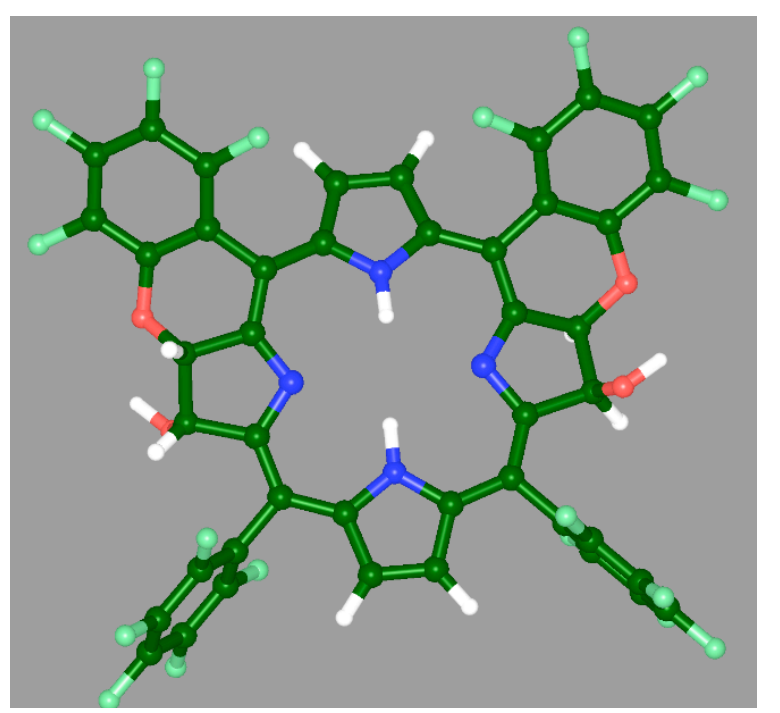

$$
\mathrm{E}=-4000.903546 \text { Hartrees }
$$

\section{2}

trans_syn_fused_oh_2_TFB_hrot_2.pdb

$\begin{array}{lrrr}\mathrm{F} & 7.29600 & 3.21200 & -0.76800 \\ \mathrm{~F} & -2.31900 & 5.39300 & 1.08400 \\ \mathrm{~F} & 2.49100 & 5.41100 & 0.48300 \\ \mathrm{~F} & 7.09900 & 5.86600 & -0.16700 \\ \mathrm{~F} & 4.64800 & 6.91700 & 0.47600 \\ \mathrm{~F} & -4.40200 & 7.05000 & 0.94600 \\ \mathrm{~F} & -6.84400 & 6.16200 & 0.09400 \\ \mathrm{~F} & -7.17600 & 3.53200 & -0.55600 \\ \mathrm{C} & 0.62700 & 4.28100 & -0.99100 \\ \mathrm{H} & 1.22600 & 5.05300 & -1.44400 \\ \mathrm{C} & 4.11200 & 0.76900 & -1.00100 \\ \mathrm{~N} & 0.02000 & 2.31600 & -0.08800 \\ \mathrm{H} & 0.06200 & 1.38100 & 0.29700 \\ \mathrm{~N} & 2.03400 & 0.14000 & -0.13400 \\ \mathrm{C} & 2.46100 & 2.57800 & -0.40500 \\ \mathrm{C} & -1.13600 & 3.04500 & -0.27300 \\ \mathrm{C} & -0.74200 & 4.27300 & -0.88200 \\ \mathrm{H} & -1.41700 & 5.04100 & -1.22600 \\ \mathrm{C} & 4.75500 & 5.63600 & 0.09200 \\ \mathrm{C} & -3.46900 & 4.90300 & 0.58100 \\ \mathrm{C} & 6.00100 & 5.10300 & -0.21200 \\ \mathrm{C} & 1.12600 & 3.05500 & -0.45500 \\ \mathrm{C} & -4.87200 & 3.11400 & -0.17000 \\ \mathrm{C} & -2.43800 & 2.60600 & 0.06800 \\ \mathrm{C} & 3.64500 & 3.48000 & -0.37900 \\ \mathrm{C} & 2.78300 & 1.20700 & -0.43000 \\ \mathrm{C} & 6.08900 & 3.75600 & -0.53600 \\ \mathrm{C} & 3.62200 & 4.83000 & 0.03800 \\ \mathrm{C} & 4.30200 & -0.61200 & -0.37300 \\ \mathrm{C} & -3.56800 & 3.56600 & 0.15600 \\ \mathrm{C} & 4.94900 & 2.94900 & -0.58700\end{array}$




\begin{tabular}{|c|c|c|c|}
\hline C & -4.55100 & 5.77900 & 0.54500 \\
\hline C & -2.81500 & 1.26100 & 0.21000 \\
\hline C & -5.95800 & 3.98600 & -0.21600 \\
\hline C & -5.79800 & 5.32700 & 0.12400 \\
\hline F & -3.13400 & -3.77200 & -2.18500 \\
\hline F & -5.05200 & -5.67200 & -2.28700 \\
\hline F & 3.50400 & -3.19900 & 2.54400 \\
\hline F & -4.15000 & -3.00600 & 2.38600 \\
\hline $\mathrm{F}$ & -6.52600 & -6.25600 & -0.06300 \\
\hline F & -6.06300 & -4.91400 & 2.27300 \\
\hline F & 5.41700 & -5.10000 & 2.73300 \\
\hline F & 3.56800 & -3.61600 & -2.18100 \\
\hline F & 6.41100 & -6.27500 & 0.47700 \\
\hline F & 5.47600 & -5.52600 & -1.98000 \\
\hline $\mathrm{C}$ & -0.73800 & -4.08000 & 0.37300 \\
\hline $\mathrm{H}$ & -1.38600 & -4.93700 & 0.47100 \\
\hline $\mathrm{N}$ & -0.04900 & -1.95300 & 0.14200 \\
\hline $\mathrm{H}$ & -0.05700 & -0.94700 & 0.02400 \\
\hline $\mathrm{N}$ & -2.07600 & 0.14500 & 0.15700 \\
\hline C & -2.51300 & -2.26600 & 0.15700 \\
\hline C & 1.08100 & -2.73400 & 0.20900 \\
\hline C & 0.64100 & -4.08000 & 0.36800 \\
\hline $\mathrm{H}$ & 1.29000 & -4.93800 & 0.45700 \\
\hline $\mathrm{C}$ & -5.34000 & -4.62900 & 1.18000 \\
\hline C & 3.97600 & -3.74900 & 1.41600 \\
\hline C & -5.57800 & -5.31300 & -0.01000 \\
\hline C & -1.17800 & -2.73300 & 0.22600 \\
\hline $\mathrm{C}$ & 4.00400 & -3.96800 & -0.95600 \\
\hline $\mathrm{C}$ & 2.41600 & -2.28100 & 0.07700 \\
\hline $\mathrm{C}$ & -3.57500 & -3.32200 & 0.10400 \\
\hline C & -2.91000 & -0.93500 & 0.11800 \\
\hline $\mathrm{C}$ & -4.82400 & -5.01500 & -1.14200 \\
\hline C & -4.34900 & -3.65100 & 1.22100 \\
\hline $\mathrm{C}$ & 3.47400 & -3.34000 & 0.17600 \\
\hline C & -3.83900 & -4.03100 & -1.07500 \\
\hline C & 4.95800 & -4.73100 & 1.52900 \\
\hline C & 2.83100 & -0.97500 & -0.15000 \\
\hline $\mathrm{C}$ & 4.98800 & -4.95000 & -0.87200 \\
\hline $\mathrm{C}$ & 5.46700 & -5.33200 & 0.38000 \\
\hline C & -4.37300 & -0.51600 & -0.06200 \\
\hline 0 & -4.70100 & -0.66700 & -1.43800 \\
\hline C & -4.28600 & 0.95000 & 0.37700 \\
\hline 0 & -5.10800 & 1.79500 & -0.43600 \\
\hline $\mathrm{H}$ & -5.38800 & -0.02300 & -1.66500 \\
\hline $\mathrm{H}$ & -5.07200 & -1.08200 & 0.56100 \\
\hline $\mathrm{H}$ & -4.59800 & 1.06000 & 1.42700 \\
\hline $\mathrm{H}$ & 3.98400 & 0.65100 & -2.09000 \\
\hline $\mathrm{H}$ & 4.81700 & -1.30000 & -1.05100 \\
\hline 0 & 5.21500 & 1.62700 & -0.77500 \\
\hline 0 & 4.95400 & -0.56100 & 0.88900 \\
\hline $\mathrm{H}$ & 5.78100 & -0.06600 & 0.78400 \\
\hline
\end{tabular}




\section{Optimized coordinates of compound $11^{\mathrm{H}}-$ Z-anti}

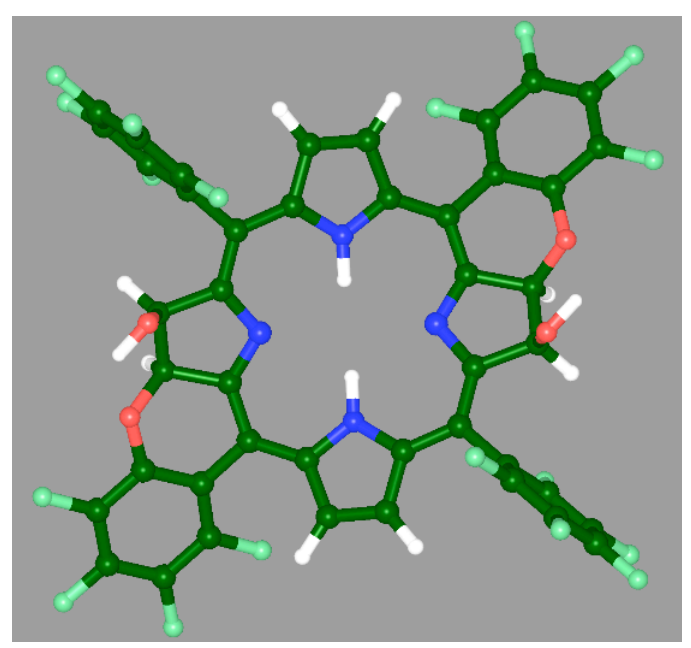

$\begin{array}{lrrr}\text { E = -4000.917703 Hartrees } & & \\ \text { 82 } & & & \\ \text { Cis_anti_fused_oh_2 TFB.pdb } & \\ \text { F } & 3.18100 & -2.69800 & 3.10500 \\ \text { F } & 5.25800 & -4.18100 & 3.99700 \\ \text { F } & -1.46500 & -5.23100 & -2.26800 \\ \text { F } & 4.79900 & -3.11700 & -1.33400 \\ \text { F } & 7.11200 & -5.14300 & 2.23700 \\ \text { F } & 6.87000 & -4.60600 & -0.43200 \\ \text { F } & -3.30900 & -7.14000 & -2.56900 \\ \text { F } & -5.84500 & -6.79500 & -1.60500 \\ \text { F } & -6.52300 & -4.45500 & -0.37800 \\ \text { C } & 1.29000 & -4.10700 & 0.32000 \\ \text { H } & 2.01400 & -4.84200 & 0.63800 \\ \text { C } & 4.36400 & -0.01200 & 0.28600 \\ \text { N } & 0.36500 & -2.13600 & -0.22400 \\ \text { H } & 0.23600 & -1.13900 & -0.34800 \\ \text { N } & 2.04200 & 0.23000 & -0.24100 \\ \text { C } & 2.76900 & -2.02800 & 0.36800 \\ \text { C } & -0.61700 & -3.08700 & -0.37200 \\ \text { C } & -0.02300 & -4.33800 & -0.02700 \\ \text { H } & -0.52800 & -5.29100 & -0.01900 \\ \text { C } & 5.96100 & -4.13400 & 0.43300 \\ \text { C } & -2.67500 & -5.02300 & -1.71100 \\ \text { C } & 6.08600 & -4.40800 & 1.79300 \\ \text { C } & 1.54600 & -2.71000 & 0.18300 \\ \text { C } & -4.30100 & -3.65300 & -0.60900 \\ \text { C } & -1.96600 & -2.78000 & -0.68800 \\ \text { C } & 3.92000 & -2.85600 & 0.85600 \\ \text { C } & 2.99200 & -0.67400 & 0.11900 \\ \text { C } & 5.13900 & -3.91700 & 2.68900 \\ \text { C } & 4.88600 & -3.36900 & -0.01400 \\ \text { C } & 4.14100 & 1.25700 & -0.55000 \\ \text { C } & -2.95300 & -3.84100 & -1.00600 \\ \text { C } & 4.07500 & -3.15100 & 2.21500\end{array}$




\begin{tabular}{|c|c|c|c|}
\hline C & -3.62700 & -6.02100 & -1.90000 \\
\hline $\mathrm{C}$ & -2.52400 & -1.50200 & -0.54900 \\
\hline $\mathrm{C}$ & -5.25900 & -4.65000 & -0.78900 \\
\hline C & -4.92200 & -5.84400 & -1.42000 \\
\hline F & -3.13500 & 2.64700 & 3.01000 \\
\hline F & -5.22800 & 4.14200 & 3.84300 \\
\hline F & -4.67000 & 3.00600 & -1.46400 \\
\hline $\mathrm{F}$ & -7.04800 & 5.08000 & 2.03600 \\
\hline $\mathrm{F}$ & -6.75700 & 4.50700 & -0.62000 \\
\hline $\mathrm{F}$ & 6.63200 & 4.35700 & -0.31700 \\
\hline F & 1.61100 & 5.10700 & -2.31000 \\
\hline F & 5.97800 & 6.68100 & -1.58800 \\
\hline F & 3.46100 & 7.01200 & -2.60300 \\
\hline C & -1.19200 & 4.01800 & 0.24100 \\
\hline $\mathrm{H}$ & -1.92200 & 4.75800 & 0.53600 \\
\hline $\mathrm{N}$ & -0.25800 & 2.04000 & -0.25800 \\
\hline $\mathrm{H}$ & -0.12600 & 1.04200 & -0.36600 \\
\hline $\mathrm{N}$ & -1.93400 & -0.32600 & -0.27500 \\
\hline C & -2.67200 & 1.94000 & 0.29000 \\
\hline C & 0.72800 & 2.99000 & -0.40100 \\
\hline C & 0.12700 & 4.24500 & -0.08400 \\
\hline $\mathrm{H}$ & 0.63200 & 5.19700 & -0.08000 \\
\hline C & -5.86400 & 4.04700 & 0.26800 \\
\hline C & 4.41500 & 3.55300 & -0.57800 \\
\hline $\mathrm{C}$ & -6.01400 & 4.33900 & 1.62200 \\
\hline C & -1.44500 & 2.61900 & 0.11900 \\
\hline C & 2.81000 & 4.90700 & -1.72800 \\
\hline C & 2.08100 & 2.67800 & -0.68800 \\
\hline $\mathrm{C}$ & -3.83100 & 2.77400 & 0.74600 \\
\hline C & -2.89100 & 0.58300 & 0.05600 \\
\hline C & -5.08400 & 3.86000 & 2.54100 \\
\hline C & -4.78200 & 3.27600 & -0.14900 \\
\hline C & 3.07500 & 3.73500 & -1.00200 \\
\hline C & -4.01200 & 3.08800 & 2.09700 \\
\hline C & 5.37700 & 4.54700 & -0.75300 \\
\hline C & 2.63700 & 1.40200 & -0.52000 \\
\hline C & 3.76600 & 5.90200 & -1.91300 \\
\hline C & 5.05200 & 5.73200 & -1.40700 \\
\hline $\mathrm{C}$ & -4.26500 & -0.07700 & 0.20600 \\
\hline 0 & -4.48300 & -0.33200 & 1.58800 \\
\hline C & -4.02700 & -1.35800 & -0.60800 \\
\hline 0 & -4.70600 & -2.48000 & -0.03700 \\
\hline $\mathrm{H}$ & -5.07500 & -1.09400 & 1.67200 \\
\hline $\mathrm{H}$ & -5.08500 & 0.51900 & -0.20800 \\
\hline $\mathrm{H}$ & -4.37400 & -1.22300 & -1.64400 \\
\hline 0 & 4.55600 & 0.26100 & 1.66800 \\
\hline $\mathrm{H}$ & 5.19100 & -0.61400 & -0.10400 \\
\hline $\mathrm{H}$ & 5.14700 & 1.02500 & 1.75200 \\
\hline $\mathrm{H}$ & 4.50600 & 1.10800 & -1.57700 \\
\hline 0 & 4.81000 & 2.38700 & 0.01800 \\
\hline
\end{tabular}




\section{Optimized coordinates of compound $11^{\mathrm{H}}-Z-s y n$}

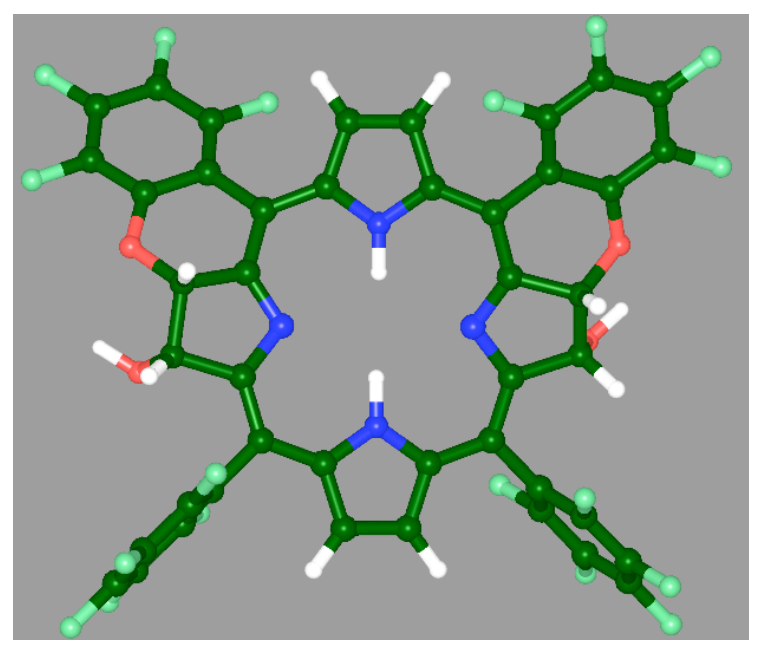

$\begin{array}{lrrr}\text { E = -4000.905199 Hartrees } & & \\ \text { 82 } & & & \\ \text { Cis_syn_fused_oh_2_TFB_hrot_2.pdb } & \\ \text { F } & -6.84700 & 3.80400 & -0.46900 \\ \text { F } & 2.91100 & 4.60700 & 2.47400 \\ \text { F } & -1.80000 & 4.98700 & -2.10100 \\ \text { F } & -6.38000 & 5.96600 & -2.06400 \\ \text { F } & -3.81800 & 6.51300 & -2.87700 \\ \text { F } & 5.18100 & 5.97300 & 2.80500 \\ \text { F } & 7.50400 & 5.12900 & 1.63300 \\ \text { F } & 7.52200 & 2.82700 & 0.16800 \\ \text { C } & -0.15100 & 4.45400 & -0.03100 \\ \text { H } & -0.67900 & 5.38100 & -0.17600 \\ \text { C } & -3.90700 & 1.38600 & 0.87600 \\ \text { N } & 0.29400 & 2.26100 & 0.18300 \\ \text { H } & 0.20700 & 1.25200 & 0.16700 \\ \text { N } & -1.91900 & 0.31600 & 0.25400 \\ \text { C } & -2.08200 & 2.75700 & -0.20100 \\ \text { C } & 1.47300 & 2.92900 & 0.42400 \\ \text { C } & 1.17300 & 4.32400 & 0.29500 \\ \text { H } & 1.87500 & 5.13200 & 0.42700 \\ \text { C } & -4.05700 & 5.50200 & -2.02800 \\ \text { C } & 4.00300 & 4.17300 & 1.81000 \\ \text { C } & -5.36000 & 5.21600 & -1.63400 \\ \text { C } & -0.73100 & 3.14300 & -0.10000 \\ \text { C } & 5.18400 & 2.57100 & 0.48100 \\ \text { C } & 2.71100 & 2.28700 & 0.64000 \\ \text { C } & -3.17800 & 3.64700 & -0.66100 \\ \text { C } & -2.54400 & 1.48700 & 0.22800 \\ \text { C } & -5.58600 & 4.12200 & -0.80900 \\ \text { C } & -3.00900 & 4.71300 & -1.56900 \\ \text { C } & -4.24100 & -0.10400 & 0.72700 \\ \text { C } & 3.94300 & 3.04000 & 0.98300 \\ \text { C } & -4.52900 & 3.33000 & -0.35200 \\ \text { C } & 5.18000 & 4.88600 & 2.01800\end{array}$




\begin{tabular}{|c|c|c|c|}
\hline C & 2.93300 & 0.92100 & 0.37500 \\
\hline $\mathrm{C}$ & 6.36600 & 3.28200 & 0.67900 \\
\hline C & 6.36700 & 4.45200 & 1.43300 \\
\hline$F$ & 4.08200 & -3.82500 & 1.24500 \\
\hline F & 5.70100 & -5.81900 & 0.39700 \\
\hline$F$ & -3.97100 & -3.42000 & -1.48200 \\
\hline F & 2.33600 & -3.39200 & -3.14500 \\
\hline $\mathrm{F}$ & 5.65100 & -6.60600 & -2.22000 \\
\hline$F$ & 3.96300 & -5.38200 & -3.98300 \\
\hline $\mathrm{F}$ & -6.02700 & -5.10800 & -1.01000 \\
\hline F & -3.55200 & -2.64100 & 3.17900 \\
\hline F & -6.85600 & -5.57900 & 1.54900 \\
\hline F & -5.60700 & -4.33800 & 3.64000 \\
\hline $\mathrm{C}$ & 0.34900 & -4.12500 & -0.15200 \\
\hline $\mathrm{H}$ & 0.90000 & -5.03800 & -0.32300 \\
\hline $\mathrm{N}$ & -0.12600 & -1.94600 & 0.07400 \\
\hline $\mathrm{H}$ & -0.04000 & -0.93800 & 0.08000 \\
\hline $\mathrm{N}$ & 2.06900 & -0.05400 & 0.08400 \\
\hline C & 2.24500 & -2.43800 & -0.46600 \\
\hline C & -1.29400 & -2.63000 & 0.30800 \\
\hline C & -0.98900 & -4.01300 & 0.18300 \\
\hline $\mathrm{H}$ & -1.69200 & -4.82100 & 0.31700 \\
\hline C & 3.98600 & -4.99500 & -2.70100 \\
\hline C & -4.35300 & -3.62400 & -0.21400 \\
\hline C & 4.84800 & -5.62100 & -1.80300 \\
\hline C & 0.89700 & -2.81500 & -0.21200 \\
\hline C & -4.14300 & -3.24000 & 2.12700 \\
\hline C & -2.55400 & -2.02900 & 0.57500 \\
\hline C & 3.15400 & -3.53800 & -0.92300 \\
\hline C & 2.77400 & -1.16900 & -0.29900 \\
\hline C & 4.87200 & -5.21800 & -0.47000 \\
\hline C & 3.15500 & -3.96900 & -2.25500 \\
\hline C & -3.69100 & -2.97200 & 0.83100 \\
\hline C & 4.03000 & -4.19200 & -0.05100 \\
\hline C & -5.41300 & -4.50000 & 0.01400 \\
\hline C & -2.82600 & -0.67100 & 0.57100 \\
\hline C & -5.20100 & -4.10800 & 2.38300 \\
\hline C & -5.83900 & -4.74100 & 1.31800 \\
\hline C & 4.24800 & -0.82900 & -0.53600 \\
\hline $\mathrm{C}$ & 4.36000 & 0.42300 & 0.34300 \\
\hline $\mathrm{H}$ & -3.78200 & 1.62400 & 1.94400 \\
\hline $\mathrm{H}$ & -4.76400 & -0.49100 & 1.60900 \\
\hline 0 & -4.91900 & 2.23500 & 0.35800 \\
\hline O & -4.97100 & -0.38900 & -0.45900 \\
\hline $\mathrm{H}$ & -5.74200 & 0.19800 & -0.48900 \\
\hline 0 & 4.41800 & -0.54900 & -1.92000 \\
\hline $\mathrm{H}$ & 4.93900 & -1.61400 & -0.21500 \\
\hline $\mathrm{H}$ & 5.18500 & 0.03400 & -2.02400 \\
\hline $\mathrm{H}$ & 4.70600 & 0.14900 & 1.35200 \\
\hline 0 & 5.25800 & 1.39100 & -0.20700 \\
\hline
\end{tabular}

